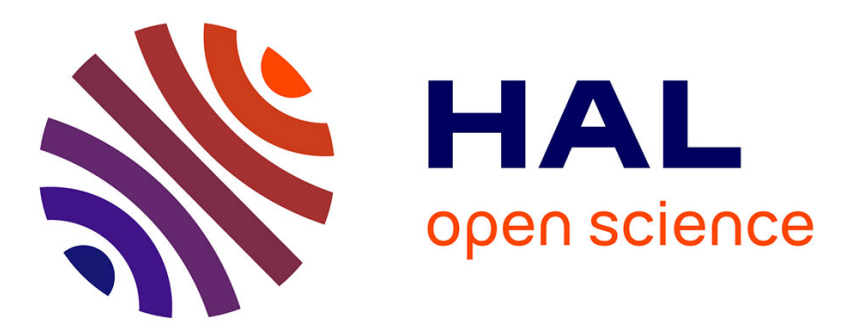

\title{
Combustion chemical kinetics of biodiesel and related compounds (methyl and ethyl esters): Experiments and modeling - Advances and future refinements
}

Lucie Coniglio, Hayet Hayet Bennadji, Pierre Alexandre Glaude, Olivier Herbinet, Francis Billaud

\section{To cite this version:}

Lucie Coniglio, Hayet Hayet Bennadji, Pierre Alexandre Glaude, Olivier Herbinet, Francis Billaud. Combustion chemical kinetics of biodiesel and related compounds (methyl and ethyl esters): Experiments and modeling - Advances and future refinements. Progress in Energy and Combustion Science, 2013, 39, pp.340-382. 10.1016/j.pecs.2013.03.002 . hal-00846052

\section{HAL Id: hal-00846052 https://hal.science/hal-00846052}

Submitted on 18 Jul 2013

HAL is a multi-disciplinary open access archive for the deposit and dissemination of scientific research documents, whether they are published or not. The documents may come from teaching and research institutions in France or abroad, or from public or private research centers.
L'archive ouverte pluridisciplinaire HAL, est destinée au dépôt et à la diffusion de documents scientifiques de niveau recherche, publiés ou non, émanant des établissements d'enseignement et de recherche français ou étrangers, des laboratoires publics ou privés. 


\title{
Combustion chemical kinetics of biodiesel and related compounds (methyl and ethyl esters): Experiments and modeling - Advances and future refinements
}

\author{
Lucie Coniglio*, Hayet Bennadji, Pierre Alexandre Glaude, Olivier Herbinet, \\ Francis Billaud
}

Université de Lorraine, École Nationale Supérieure des Industries Chimiques de Nancy, Laboratoire Réactions et Génie des Procédés, UMR CNRS 7274, 1 rue Grandville BP 20451, 54001 Nancy Cedex, France

\begin{abstract}
The motivation for and challenges in reducing the world's dependence on crude oil while simultaneously improving engine performance through better fuel efficiency and reduced exhaust emissions have led to the emergence of new fuels and combustion devices. Over the past ten years, considerable effort has gone into understanding combustion phenomena in relation to emerging fuel streams entering the market. The present article focuses specifically on one typical emerging transportation fuel dedicated to the diesel engine, biodiesel, with an emphasis on ethyl esters because of recently renewed interest in its use as a completely green biofuel. Based on a review of the research developments over the past ten years in advanced experimental and kinetic modeling related to the oxidation of biodiesel and related components, the main gaps in the field are highlighted to facilitate the convergence toward clean and efficient combustion in diesel engines. After briefly outlining the synergy between "feedstocks - conversion process - biodiesel combustion", the combustion kinetics of methyl and ethyl biodiesels are reviewed with emphasis on two complementary aspects: mechanism generation based on a detailed chemical kinetic approach that leads to predictive combustion models and experimental combustion devices that generate the data required during the development and validation of the predictive models.
\end{abstract}

Keywords: Methyl and ethyl biodiesels; Combustion; Diesel engine; Performance and emission; Chemical kinetics of oxidation; Experiments and modeling

* Lucie Coniglio

Adress: Université de Lorraine, École Nationale Supérieure des Industries Chimiques de Nancy, Laboratoire Réactions et Génie des Procédés, UMR CNRS 7274, 1 rue Grandville BP 20451, 54001

Nancy Cedex, France

Tel.: +33 383175 025; fax: +33 383322975 .

E-mail: lucie.coniglio@univ-lorraine.fr 


\section{Introduction}

Energy demand around the world is continuously increasing, including for petroleum-based energy. Petroleum is the single largest energy resource that has been consumed by the world's population, exceeding natural gas, coal, nuclear energy and renewable materials. According to the International Energy Outlook of 2011, which was published by the U.S. Energy Information Administration [2], the world use of liquid fuels will increase from 85.7 million barrels per day in 2008 to 112.2 million barrels per day in 2035. In addition, the transport sector will account for $82 \%$ of the total increase in liquid fuel use, with the remaining growth attributed to the industrial sector [2]. Because of the progressive depletion of oil resources in combination with increasing energy consumption and the negative environmental impact of fossil fuel use, there has been a shift toward alternative sources of energy that are renewable, sustainable, efficient, cost-effective and generate reduced emissions [3] and [4]. Biofuels, especially bioethanol and biodiesel, are among the most viable liquid transportation fuels for the foreseeable future and can contribute significantly to sustainable development in terms of socioeconomic and environmental concerns. Liquid biofuels are manufactured from biomass that is mainly derived from agriculture resources (sugar- or grain-based for bioethanols and oilseed-based for biodiesels) [5]. Thus, this natural resource is more evenly distributed geographically than fossil fuels, which provides developed and developing nations with energy supply independence and security, local populations with employment opportunities and rural communities with modern energy [4] and [6]. In addition, biofuels can be used in blends with conventional fossil fuels with no or very little engine modification. Biodiesel is blended with petrodiesel (petroleum diesel, also called fossil diesel) for use in compression-ignition engines, whereas bioethanol is blended with gasoline for use in spark-ignition engines. Regarding environmental concerns, the use of biomass-based energy contributes to a significant reduction in greenhouse gas (GHG) emissions. Biodiesel and bioethanol (when not produced from corn) are considered "carbon-neutral" fuels because of the equal balance between the carbon dioxide $\left(\mathrm{CO}_{2}\right)$ released during combustion and that absorbed during the photosynthesis of the raw material used to manufacture the fuels [7]. Furthermore, both biofuels are aromatic-free and lead to a $90 \%$ reduction in cancer risk [8].

Biodiesel ("bio" from life in Greek and "diesel" from Dr. Rudolf Diesel, German engineer, who invented the diesel engine which is able to run on a host of fuels including coal dust suspended in water, heavy mineral oil, and vegetable oils) is typically produced through the conversion of biolipids with methanol, yielding fatty acid methyl esters (FAME) as biodiesel and glycerol as a by-product. Although methanol has been preferred to bioethanol in industrial applications because of its lower cost [9], biodiesel is $100 \%$ renewable only when the alcohol used in the conversion process is also renewable (such as bioethanol); this proportion is reduced to approximately $90 \mathrm{wt} \%$ when a fossil alcohol, such as methanol, is used [10]. Furthermore, bioethanol is less toxic, less corrosive and less volatile than methanol, providing thus a safer work environment. In addition, bioethanol is already produced in large quantities in some countries, where it is recommended as transportation fuel [11]. Thus, using bioethanol to produce biodiesel in the form of fatty acid ethyl esters (FAEE) would further enhance the sustainability of new biofuels. In the future, biomethanol (i.e. renewable methanol) could be produced from biomass, either by fermentation or thermal conversion, but the last process being the most promising has to be optimized to become economically viable [12]. For the time being, biodiesel is often assimilated to FAME; however, discussions are underway to include FAEE in its definition [13].

In addition, industrial-scale biodiesel and bioethanol production primarily uses edible agricultural products (rapeseed and soybean crops for biodiesel and sugarcane, wheat, and corn crops for bioethanol). This feature represents a significant weakness in terms of the sustainability of this class of so-called first-generation (1G) biofuels. The limitation of agricultural biomass resources has induced negative outcomes in several ways: socioeconomically due to competition with food crops 
and environmentally due to deforestation (leading to a biodiversity reduction) and a shift of pollution (with $\mathrm{CO}_{2}$ consumption by plant photosynthesis compensated by water, air and soil pollution during production and conversion of the resources into biofuels) $[14,15]$. To this end, alternative feedstocks that do not compete with food crops have been investigated [4,16-18]. Forest and agricultural residues (molasses, grape marcs, or even lignocellulosic residues from the palm oil industry) have been used successfully to produce bioethanol $[19,20]$. Biolipids have been derived from various resources for the production of biodiesel, including non-edible oils from readily available and sustainable plant biomass, such as Jatropha, Karanja, Mahua or Neem [21-23], and waste cooking oil and animal fats [24-27] or microalgae [28,29]. Such raw materials with adequate conversion processes, which generate so-called second-generation $(2 \mathrm{G})$ and third-generation biofuels (3G, for microalgae), have the significant benefits of decreasing GHG emissions and the total production cost (70-95\% of the reductions are due to the raw material cost in biodiesel production) [30,31]. Furthermore, the diversification of biomass-derived feedstocks and conversion technologies represents a potential mainstay for the long-term security of the supply of sustainable biofuels [32].

The evolution toward alternative transportation fuels necessitates a reevaluation of the adequacy of current engines in terms of performance and emission requirements. Within this context, the United States Department of Energy (DOE) identified an overarching challenge for the 21st century in the field of emerging fuels and engine technologies [33]: "The development of a validated, predictive, multi-scale, combustion modeling capability to optimize the design and operation of evolving fuels in advanced engines for transportation applications". Kinetic models with previously established robustness (based on both experiment and theory) built to accurately predict the properties and combustion behavior of virtually any fuel will allow the efficient evaluation of "fuel-engine" systems through simulation. These advances will permit fuel formulation to be properly defined for a given engine technology (by assessing the influence of additives or new functional groups on fuel performance and emission) and the development of fuel-flexible engine designs with the objective of minimizing emissions while optimizing efficiency [33].

Therefore, a state-of-the-art review of the kinetics of biodiesel combustion was necessary to highlight recent advances, remaining difficulties and future progress. Few review papers have been published on this subject. Kohse-Höinghaus et al. [34] focused on various biofuel combustion chemistries from bioethanol to biodiesel, with particular attention to the analysis of the species composition of laminar premixed flames by molecular beam mass spectrometry and the development of appropriate combustion models. Later, Lai et al. [35] conducted a complementary study that focused on biodiesel fuels with a thorough and critical synthesis of the advances in chemical kinetic modeling related to biodiesel combustion. The present review is specifically concerned with biodiesel fuels and aims to expand on the two aforementioned works by highlighting (i) the experimental aspects of the combustion kinetics that were essential in the development of theory and successful modeling; (ii) FAEE studies, which are much less numerous than FAME studies, and (iii) the pyrolysis of the two classes of esters (FAME and FAEE), as this pure thermal process is an integral part of the combustion kinetics at high temperatures. Furthermore, important studies that provide key research results and conclusions in relation with combustion of biodiesel fuels and that were published after the reviews by Kohse-Höinghaus et al. [34] and Lai et al. [35] will be covered to review the most recent advances. Before addressing the core subject of the manuscript that is the biodiesel combustion kinetics with the outstanding issues and future objectives, the synergy between "feedstocks - conversion process - biodiesel combustion" will be briefly outlined. The whole of this information will provide the requisite background for our conclusions regarding the necessary development of fuel-flexible engine technologies and feedstock-flexible conversion processes, and even fuel-flexible production processes, to induce resource diversification and provide a wide range of new fuels. Furthermore, the discussion regarding biodiesel combustion kinetics has been structured to reflect the timeline of the most significant advances to highlight the 
researchers' methodology. The authors have done their best to produce a satisfactory compromise between being concise and detail.

\section{Synergy between "feedstocks - conversion process - biodiesel combustion"}

To optimize the selection of next-generation alternative fuels, a wise approach is to consider the synergy existing between feedstocks, conversion process, and biodiesel combustion (the latter requiring a fine knowledge of combustion chemical kinetics in order to formulate a fuel that will lead to cleaner emissions and better engine performance) [36-38]. Indeed, the combustion behavior of a fuel is significantly influenced by its formulation (molecular structures and proportions of the components) which in turn, and particularly in case of biodiesel, is significantly influenced by the feedstocks and conversion process selected to produce it. Hence, all these aspects should be considered simultaneously to isolate a sustainable alternative fuel leading to cleaner emissions and higher performance, not only during combustion but also during production. Also, this global approach should help the use of computational tools integrating kinetic and thermodynamic models in order to orientate reliably the selected feedstocks and conversion process toward the production of a fuel with specific combustion properties (and this, while meeting the sustainability criteria along the entire chain).

The main features of the chain "feedstocks - conversion process - biodiesel combustion" are shortly outlined in the following by focusing on the impacts of resource diversification (biolipids and alcohol). An exhaustive overview of the fundamental and technical aspects at the different stages of the chain can be found in various articles and literature reviews: for the transesterification reaction $[12,16,39-74]$, biodiesel production $[4,12,17,18,36-38,42,69,70,77-97]$, biodiesel properties $[5,8,36-$ $38,56,98-106]$, and diesel engine performance and emissions of biodiesel fuel $[8,10,36,37,101,107-$ 139] (these citations are not exhaustive lists).

\subsection{From resources to biodiesel products through transesterification}

Neat vegetable oils (or any lipid resources) are unsuitable as fuel for modern diesel engines [5]. Poor engine performance and emission characteristics, including engine failure, have been observed even when lipid resources are blended with petrodiesel $[107,108]$. One method of overcoming these issues is to chemically transform the lipid resources to bring their combustion-related properties closer to those of petrodiesel. By reducing viscosity of the lipid resources significantly, transesterification has long been the preferred method for their chemical conversion and is currently used for generating the biofuel distributed in the market for diesel engines.

As depicted by Fig. 1a, transesterification (or alcoholysis) is the reaction of triglycerides (the major components of lipid resources) with an alcohol (methanol or, by extension, ethanol) to form biodiesel (FAME or, by extension, FAEE) and glycerol (by-product). Biodiesel and glycerol are not miscible, and the latter is removed from the reaction medium by decantation. Globally, the stoichiometry for the reaction is 3:1 alcohols to triglycerides (TG), which forms 3 mol of esters (FAME or FAEE) and $1 \mathrm{~mol}$ of glycerol. However, a higher alcohol-to-triglyceride molar ratio is used in practice because of the reversibility of the reaction, while an alkali or acid catalyst is usually employed to increase the reaction rate and yield. Furthermore, the overall chemical equation is the result of three consecutive and reversible reactions that lead to the intermediate products of diglycerides (DG) and monoglycerides (MG), as shown in Fig. 1b. As glycerides (TG, DG, and MG) usually contain different aliphatic chains (R1-R3), the biodiesel product is a mixture of FAME (or FAEE) with various chain lengths and numbers of double bonds $(-\mathrm{CH}=\mathrm{CH}-)$. Table 1 presents, for certain vegetable oils and fats, the average composition of fatty acids corresponding to the fragments $\mathrm{RxCOOH}$ with $x=1,2$ or 3 of the triglycerides (Fig. 1a). Normally linked to the glycerol backbone under the triglyceride form, these fragments should be differentiated from free fatty acids 
(FFA) that may be naturally encountered in the lipid resources (with concentrations below $0.05 \mathrm{wt} \%$ in the refined edible oils). As the data in Table 1 show, the major fatty acids in vegetable oils are the saturated fatty acids palmitic (C16:0) and stearic (C18:0) as well as the unsaturated fatty acids oleic (C18:1), linoleic (C18:2), and linolenic (C18:3) [5,56]. Animal fats (including fish oils) contain longer fatty acids (up to 22 carbon atoms) with higher degree of unsaturation (up to 6 double bonds). Thus, the lipidic resources used as raw materials determine the FAME (or FAEE) composition of the biodiesel product.

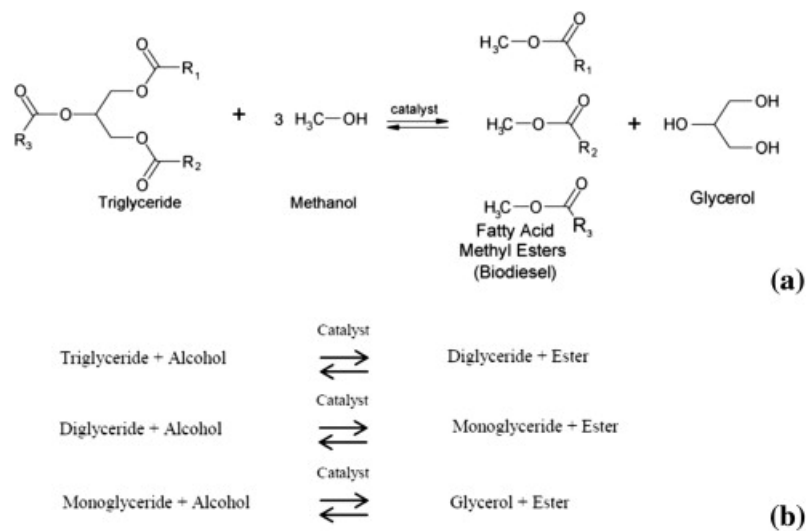

Figure 1. Transesterification reaction of triglycerides. (a) Overall chemical equation illustrated for methanolysis yielding FAME (biodiesel) and glycerol $\left(\mathrm{R}_{1}, \mathrm{R}_{2}, \mathrm{R}_{3}\right.$ are identical or different aliphatic main chains with zero to three unsaturated bond(s): $\mathrm{CH}_{3}-\left(\mathrm{CH}_{2}\right)_{\mathrm{m}}-\left(\mathrm{CH}_{2}-\mathrm{CH}=\mathrm{CH}\right)_{n}-(\mathrm{CH} 2)_{\mathrm{k}}$ with $(\mathrm{m}+\mathrm{k})=12$, $14,16,18$ or 20 , and $n=0,1,2$ or 3 (6 for fish oils) [55]). (b) Alcoholysis of triglycerides as a sequence of three consecutive and reversible reactions.

Alkaline catalysis can be used until the free fatty acid (FFA) content in the triglyceride stock is less than 1 wt\% [24]. For triglyceride stocks with up to 5 wt\% FFA, acidic catalysts have to be selected [42]. Processes based on homogeneous catalysis (mainly alkali catalysis) are the most widely used for industrial biodiesel production $[43,44]$. Other alternatives based on heterogeneous catalysis were proposed in order to avoid catalyst losses and important water consumptions when removing the catalyst from biodiesel by wet-washing [48-54]. Another alternative, recently recommended at the laboratory scale to replace efficiently the wet-washing step of biodiesel, is a treatment with an adsorbent which can be removed by filtration (such as Magnesol ${ }^{\circledR}$ or rice husk ash, a natural by-product of rice processing) $[69,79]$.

Regardless of the method used, the replacement of methanol by ethanol as feedstock causes some issues at key stages of the process: longer reaction times; the formation of more stable emulsions (when they occur), which makes the separation and purification of biodiesel and glycerol more tedious; and a greater solubility of ethanol and glycerol in the ethyl ester rich phase [62-67]. This latter point can be however countered by either evaporating the excess ethanol or adding cold glycerol $[65,66]$. Nevertheless, a much more critical issue than the aforementioned ones is the deactivation of the catalyst by the presence of water in the non-conventional feedstocks, such as crude bioethanol and $2 \mathrm{G}$ or $3 \mathrm{G}$ biolipids (non-edible oils, waste cooking oils, or microalgae). Thus, the raw materials for catalyzed transesterification reactions should have a water content below $0.06 \mathrm{wt} \%$ $[4,16,36,39]$. Emerging non-catalytic alcoholysis methods based on supercritical fluids (the alcohol with eventually a co-solvent like $\mathrm{CO}_{2}$ ) allow to avoid these problems while offering other significant advantages: short reaction times; high-grade products ( $99.8 \%$ biodiesel and $96.4 \%$ glycerol), which renders glycerol more valuable on the market and further lowers the manufacturing costs [94], [95], [96] and [97]; a flexibility in terms of feedstocks with high concentrations of FFA and water (up to 36 and $30 \mathrm{wt} \%$, respectively) $[37,55,71-76,84-86,91,92]$. The main drawback of the supercritical (SC) 
process is its high energy demand, which could be countered by the addition of $\mathrm{CO} 2$ (co-solvent) [61], [87], [88] and [93], with the integration of a heat-exchanger network [89] or the use of both biodiesel and heat power (cogeneration) [90]. Additionally, a new conceptual design of in situ generation of biodiesel fuel via SC-TG transesterification coupled with SC fuel injection and combustion was very recently proposed [37].

Table 1. Average fatty acid compositions of some vegetable oils and fats [5] and [56]. ${ }^{a}$

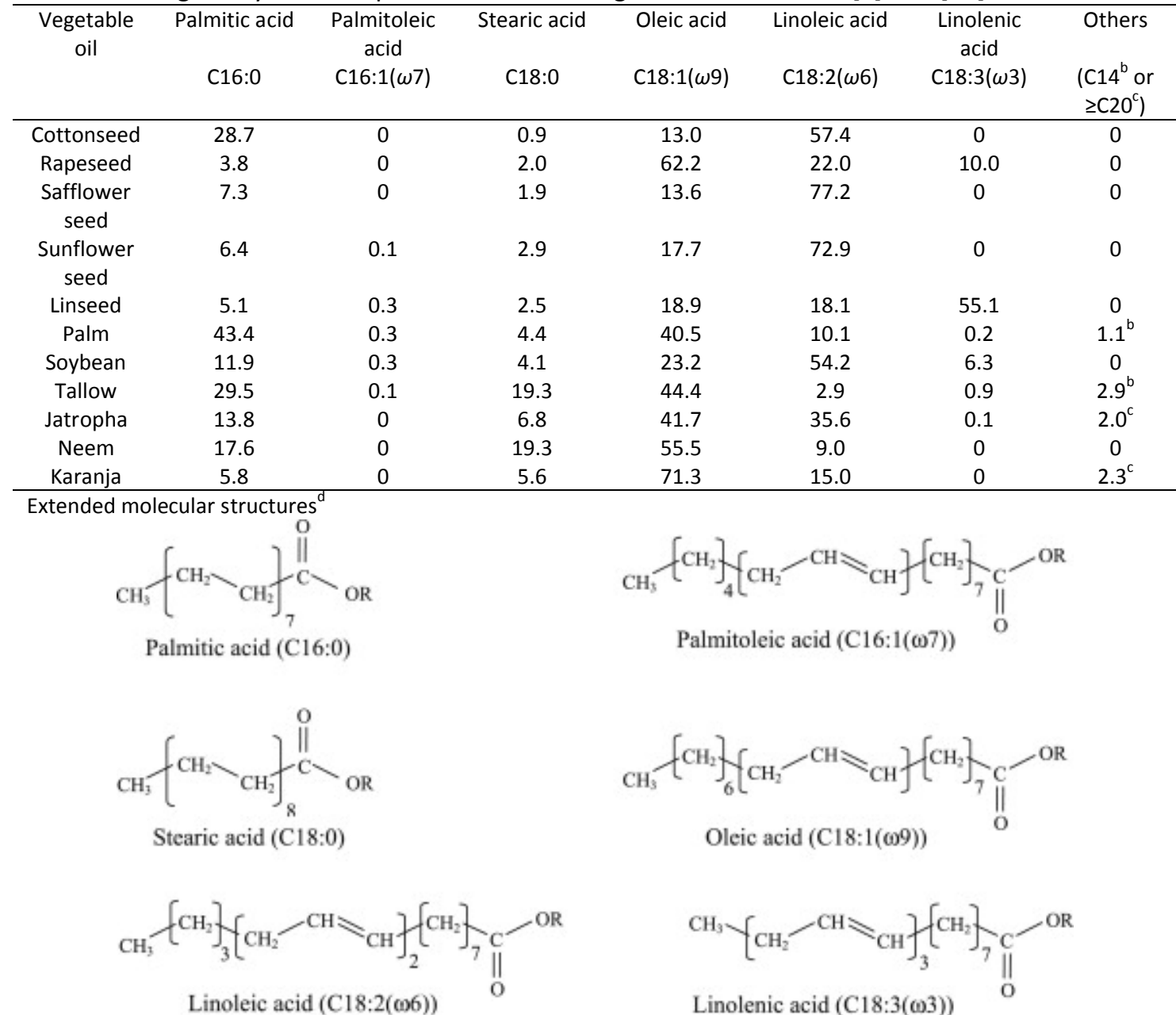

${ }^{a}$ In $C x x: n(\omega y), x x$ : number of carbon atoms; $n$ : number of double bonds; $y$ : position of the first double bond on the aliphatic main chain, starting from the extremity $\mathrm{CH}_{3}$.

${ }^{\mathrm{b}}$ Myristic acid.

${ }^{c}$ Arachidic acid.

${ }^{d}$ When $R=\mathrm{H}, \mathrm{CH}_{3}$ or $\mathrm{C}_{2} \mathrm{H}_{5}$, molecules are respectively FFA, FAME or FAEE.

In light of recent scientific and technological advances [37,55,71-75,89-93], biodiesel produced from $2 \mathrm{G}$ or $3 \mathrm{G}$ biolipids and crude bioethanol by the non-catalytic supercritical process integrating both $\mathrm{CO}_{2}$ as co-solvent and cogeneration seems a promising approach for the sustainable production of renewable energy. One particular significant feature of this alternative is that it takes advantage of the long-aliphatic chains available in the TG, which is contrary to other biofuel alternatives, such as Fischer-Tropsch (FT) diesel derived from a conversion process that cleaves the molecules available in wood materials into much smaller molecules (synthesis gas) before re-building them through another catalytic process into hydrocarbons with long-aliphatic chains. Another alternative, which is somewhere between biodiesel and FT-diesel, is the hydrodeoxygenation of lipid raw materials that 
leads to long-chain hydrocarbons, known as "renewable diesel". Knothe [38] concluded in his review that biodiesel and "renewable diesel" complement each other rather than compete. Nevertheless, the process production for the "renewable diesel" requires the use of a catalyst, which may be somewhat restrictive in terms of the nature of the lipid feedstock (particularly regarding water content).

\subsection{Key physical and thermal properties of biodiesels as fuels - quality specifications}

The properties of the produced biodiesel must adhere with specifications such as the American ASTM D6751 standard or the European EN 14214 standard. As illustrated in Table 2, which presents the ranges of the most significant quality specifications for biodiesel and petrodiesel, the fuels are very similar in some ways and quite different in others. This highlights why biodiesel is a strong candidate for replacing petrodiesel as well as the advantages and drawbacks of each fuel type. General features related to the physical and thermal properties are summarized below [5,8,33,36,100-102], whereas the other properties related to engine emission and performance characteristics will be discussed further in the dedicated section.

Table 2. Some quality specifications related to biodiesel and petrodiesel - comparison with vegetable oils $[5,98]$.

\begin{tabular}{|c|c|c|c|c|c|c|}
\hline \multirow{2}{*}{ Specifications $^{a}$} & \multirow[t]{2}{*}{ Units } & \multirow{2}{*}{$\begin{array}{c}\text { Vegetable oils } \\
{[5]}\end{array}$} & \multicolumn{2}{|c|}{ Biodiesel } & \multicolumn{2}{|c|}{ Petrodiesel } \\
\hline & & & ASTM D6751 & EN 14214 & $\begin{array}{l}\text { ASTM } \\
\text { D975 }\end{array}$ & EN 590 \\
\hline Density $\left(15^{\circ} \mathrm{C}\right)$ & $\mathrm{kg} / \mathrm{m}^{3}$ & $902-946$ & 880 & $860-900$ & 850 & $820-845$ \\
\hline $\begin{array}{l}\text { Kinematic viscosity } \\
\left(40^{\circ} \mathrm{C}\right)\end{array}$ & $\mathrm{cSt}\left(\mathrm{mm}^{2} / \mathrm{s}\right)$ & $22-54$ & $1.9-6.0$ & $3.5-5.0$ & 2.6 & $2.0-4.5$ \\
\hline Flash point & ${ }^{\circ} \mathrm{C}$ & $150-293$ & Min. $100-170$ & $>120$ & $60-80$ & $>55$ \\
\hline Cetane number & & $35-49$ & Min. 47 & Min. 51 & $40-55$ & Min. 51 \\
\hline Cloud point & ${ }^{\circ} \mathrm{C}$ & -3.9 to 31 & -3 to -12 & - & -20 & -20 to -5 \\
\hline Pour point & ${ }^{\circ} \mathrm{C}$ & -40 to 6.7 & -15 to -16 & - & -35 & - \\
\hline Lower heating value & $\mathrm{MJ} / \mathrm{kg}$ & $39-50$ & - & 35 & $42-46$ & - \\
\hline Water content & $\begin{array}{l}\%(\mathrm{v} / \mathrm{v}) \\
\operatorname{or}(\mathrm{mg} / \mathrm{kg})\end{array}$ & $\mathrm{n} / \mathrm{a}$ & $\begin{array}{l}\text { Max. } 0.05 \% \\
\text { (v/v) }\end{array}$ & $\begin{array}{l}\text { Max. } 500 \\
(\mathrm{mg} / \mathrm{kg})\end{array}$ & $\begin{array}{l}0.05 \% \\
(v / v)\end{array}$ & $\begin{array}{l}\text { Max. } 200 \\
\text { (mg/kg) }\end{array}$ \\
\hline Acid number & $\mathrm{mg} \mathrm{KOH/g}$ & $\mathrm{n} / \mathrm{a}$ & Max. 0.50 & Max. 0.50 & 0.062 & - \\
\hline Ester content & $\begin{array}{l}\%(\mathrm{~m} / \mathrm{m}) \text { or } \% \\
(\mathrm{v} / \mathrm{v})\end{array}$ & $\mathrm{n} / \mathrm{a}$ & - & $\begin{array}{l}\geq 96.5 \% \\
(\mathrm{~m} / \mathrm{m})\end{array}$ & - & $\begin{array}{l}\text { Max. 5\% } \\
\text { (v/v) }\end{array}$ \\
\hline Total glycerin content & $\%(\mathrm{~m} / \mathrm{m})$ & $\mathrm{n} / \mathrm{a}$ & Max. 0.24 & 0.25 & - & - \\
\hline Sulfur content & $\begin{array}{l}\%(\mathrm{~m} / \mathrm{m}) \text { or } \\
(\mathrm{mg} / \mathrm{kg})\end{array}$ & $\mathrm{n} / \mathrm{a}$ & $\begin{array}{l}\text { Max. } 0.05 \% \\
(\mathrm{~m} / \mathrm{m})\end{array}$ & 10 (mg/kg) & $\begin{array}{l}0.05 \% \\
(\mathrm{~m} / \mathrm{m})\end{array}$ & $10(\mathrm{mg} / \mathrm{kg})$ \\
\hline
\end{tabular}

${ }^{a}$ Definition of most specific properties are given here, with the exception of cetane number and lower heating value which will be explained in section related to diesel engine emissions and performance of biodiesel fuels. Flash point (lowest temperature corrected to a pressure of $101.3 \mathrm{kPa}$ at which application of an ignition source causes the vapors of a specimen to ignite under the specified conditions of test, i.e. measure of residual alcohol in the B100). Cloud point (temperature at which a cloud of wax crystals first appears in a liquid when it is cooled down under conditions prescribed by the specific test method). Pour point (lowest temperature at which a liquid will pour or flow under conditions prescribed by the specific test method). Lower heating value (enthalpy of combustion by considering that water is in the vapor state in the exhaust product). Acid number (quantity of base, expressed as milligrams of potassium hydroxide per gram of sample, required to titrate a sample to a specified end point). Total glycerin content (sum of the free glycerin and bonded glycerin as glycerides). $\mathrm{n} / \mathrm{a}$ : no information available in the reviewed literature.

The most obvious advantages of biodiesel versus petrodiesel are the following:

- Biodiesel is sulfur-free (producing full compatibility with catalytic post-treatment systems).

- When blended with petrodiesel, biodiesel enhances the ignition quality (through a higher cetane number) and lubricity characteristics of petrodiesel (which is mainly a result of the polarity of the oxygenated biodiesel components, not only the FAME but also more specifically the minor components, such as the FFA, MG, and glycerol).

- Biodiesel has a higher flash point (leading to safer handling, transport, and storage). 
Regarding the main disadvantages associated with biodiesel versus petrodiesel, the following should be mentioned:

- Biodiesel has poorer low-temperature properties such as the cloud point and pour point (which can create problems in cold weather by plugging fuel filters).

- In addition to density, biodiesel also has a higher viscosity (which generally leads to poorer atomization of the fuel spray, affects the accuracy of the operation of fuel injectors, and inhibits the nebulization of fuel in the ignition chamber). This drawback is not obviated despite the transesterification process that reduces significantly the viscosity of the departure lipid resources (Table 2).

- Biodiesel is inherently less stable to air and high-temperature exposure (particularly because of the possible occurrence of polyunsaturated FAME among biodiesel components), which requires the addition of small amounts of stabilizers for long-term storage.

- Impurities, such as unreacted FFA or alcohol as well as glycerol or leftover catalyst from the production process, can accelerate engine wear or corrosion and the production of acrolein, which is a photochemical smog precursor (however, those same polar components also enhance the lubrication characteristics of biodiesel).

- All the aforementioned issues highly depend on the fatty acid profile.

Thus, it becomes clear that the "ideal" biodiesel should be a compromise of well-balanced components that satisfies the quality specifications. Ramos et al. [30] presented a triangular graph (Fig. 2) that describes the optimum concentrations of saturated, monounsaturated, and polyunsaturated FAME, leading to a biodiesel that satisfies the limits imposed by EN 14214, a standard for critical parameters such as cetane number, iodine value (measure of the total unsaturation within the given biodiesel) and CFPP (cold-filter plugging point determined using a lowtemperature filterability test). This area was characterized by a high concentration of monounsaturated FAME (such as oleic acid methyl ester, C18:1(w9)) encountered in oleic sunflower or rapeseed oil biodiesel.

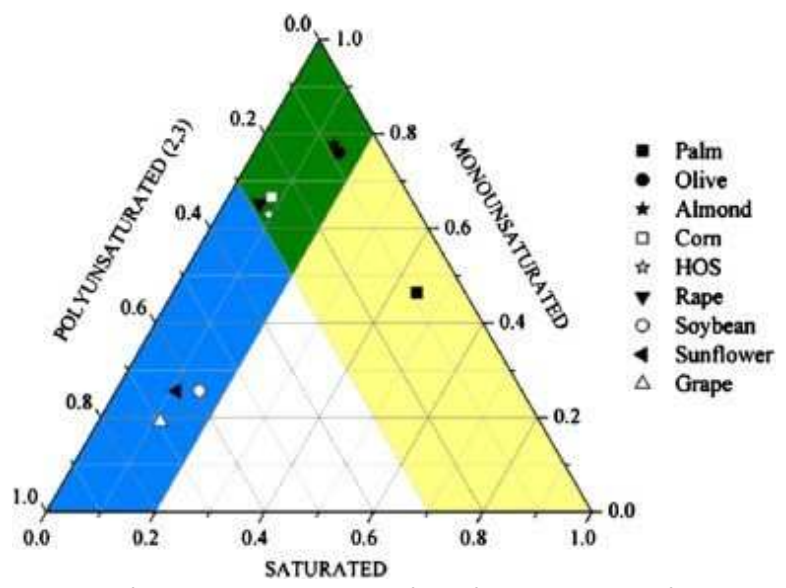

Figure 2. Biodiesel by saturated, monounsaturated, polyunsaturated FAME and areas verifying the limits imposed by European standard EN 14214 for cetane number, iodine value, and CFPP; yellow (right): good cetane number and iodine value; blue (left): good CFPP; green (intersection): biodiesel that satisfies EN 14214 standard [30].(For interpretation of the references to colour in this figure legend, the reader is referred to the web version of this article.)

\subsection{Biodiesel combustion in diesel engines}

Insight of key macroscopic features of fuel combustion as well as analysis of the diesel engine performance and emissions are necessary to understand and then predict the phenomena that 
govern them at the microscopic scale, such as fuel combustion kinetics. This aspect is addressed in this section by focusing on biodiesel fuels.

\subsubsection{Key macroscopic features of fuel combustion}

\subsubsection{Heat release rate}

An important combustion parameter linked to the chemical reaction is the heat release rate (HRR), the rate of heat emission just after ignition. Although determined indirectly during combustion in diesel engines, the HRR can provide meaningful information regarding the combustion process. As illustrated in Fig. 3 [103], the HRR history as a function of crank angle degrees may show one or two peaks, depending on the nature of the fuel and combustion conditions. In the event of two peaks, the first peak, designated as the low-temperature heat release (LTHR), corresponds to the first-stage ignition, which is representative of low-temperature oxidation, i.e. cool flame regime. The second peak, designated as the high-temperature heat release (HTHR), corresponds to the second-stage ignition, which is representative of the high-temperature combustion. In one-peak conditions, this peak may correspond to either the LTHR or HTHR phenomena.

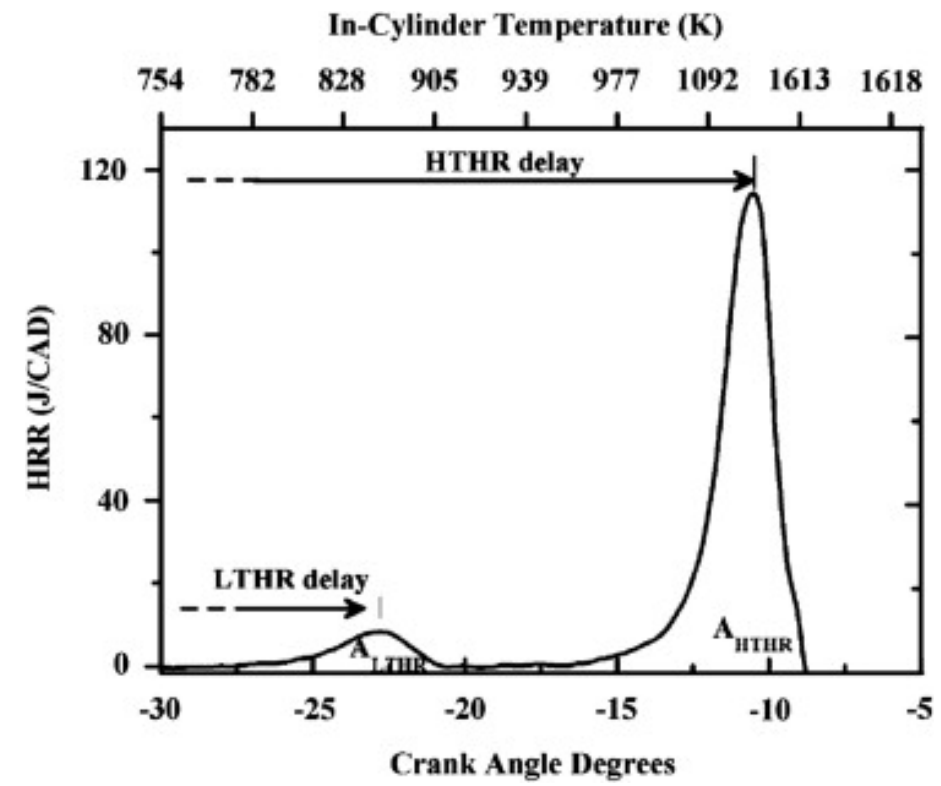

Figure 3. Definition of combustion parameters determined from heat release rate data [103]. ALTHR and AHTHR are the net energy (in J) released during the first and second stage of combustion.

\subsubsection{Cetane number and ignition delay: molecular structure-emissions relationship}

One of the most important characteristic of a diesel fuel is its ignition delay (the period between the start of injection and the start of combustion). The engine test used to characterize a fuel is the Cetane Number (CN) test, which grades autoignition quality of the diesel fuel. Fuels with a high CN will have short ignition delays (IDs). The primary reference fuels used for $\mathrm{CN}$ are cetane (n-hexadecane) with an assigned CN of 100 and 2,2,4,4,6,8,8-heptamethylnonane with an assigned $\mathrm{CN}$ of 15 . Saturated and unbranched hydrocarbons with long-aliphatic chains have high $\mathrm{CN}$ and good ignition ability as a diesel fuel compared to unsaturated or branched molecules [104]. Regarding biodiesel components, two structural effects influence the $\mathrm{CN}$ : the skeleton of the aliphatic chain (length and number of double bonds) and the nature of the alcohol moiety (methyl, ethyl, etc.). Knothe et al. [105] determined the CNs of 29 mono-alkyl esters of fatty acids using an Ignition Quality Test. The authors observed that CNs increased with chain length (as hydrocarbons), whereas 
increasing the number of double bonds or branching in the aliphatic main chain decreased CNs. Among the investigated fatty acid esters, the lowest and highest $\mathrm{CN}$ values were observed for linolenic acid and stearic acid, respectively, with CN increasing in the order linolenic < linoleic < oleic $<$ palmitic < stearic acid. If the effect of the alcohol moiety was less defined, all ethyl esters experienced higher CNs compared to the corresponding methyl esters. In addition, the esters of 2-ethylhexanol, the most $\mathrm{CH}_{2}$-rich alcohol used by the authors, displayed the highest $\mathrm{CN}$ of all tested esters.

Although engine emissions have been observed to be closely linked to $\mathrm{CN}$ and to the molecular structure of the fuel components, the relationship between emissions and $\mathrm{CN}$ is complicated by many factors, including the type of engine and the operating conditions [109]. Emissions of nitrogen oxides (NOx) and unburned hydrocarbons decreased with an increasing $\mathrm{CN}$ because of a reduction in ID times and amount of premixed fuel burned, which resulted lower average combustion temperatures and advanced combustion [110]. Nevertheless, running a diesel engine with various types of biofuels revealed that a lower $\mathrm{CN}$ and a longer ID period (higher level of premixed combustion) may increase the exergetic efficiency [111]. This finding highlights the necessity of further research for a better understanding of the relationship between macroscopic fuel properties, engine emissions and performance characteristics.

\subsubsection{Diesel engine emissions and performance of biodiesels}

Biodiesel is an oxygenated fuel containing approximately 10-15\% oxygen by weight, which results in "cleaner" combustion and improves exhaust emissions. Of all regulated emissions, nitrogen oxides (NOx) and particle matter (PM) are the most critical factors in diesel engine emissions because current technologies are close to the permitted limits, which will be even more stringent in the near future, and because new regulations (Euro 5 and 6) will consider mass- and number-based PM emissions. In addition, while current diesel engine technologies should be able to meet the future limits for regulated emissions (e.g. CO, total hydrocarbons), this is not guaranteed for some nonregulated specific emissions, such as polycyclic aromatic hydrocarbons (PAH) and carbonyl compounds, which raises concerns because of their hazards to humans and the environment. Assessment of biodiesel emissions and their impact is required before expanding the availability of biodiesels in the fuel market. The trends emerging from the reviewed literature detailed below regarding engine performance and emissions of biodiesel (FAME and FAEE) and petrodiesel are summarized in Table 3. If a wide range of diesel engines was tested, few tests were conducted using actual vehicles [112-116].

\subsubsection{FAME-biodiesel versus petrodiesel}

Recent reviews summarized scientific work on combustion performance and emission of biodiesel fuels in diesel engines [33,117], compared to conventional petrodiesel fuels [10] or focused on the separation and purification technologies that lead to high-quality biodiesel and its effects on diesel engines [36]. Most researchers have reported that the diesel engine combustion of biodiesel fuels (irrespective of feedstock) results in a decrease in particulate matter (PM), unburned hydrocarbons (UHC), sulfur oxides (SOx), volatile organic compounds (VOCs), and carbon monoxide (CO) emissions compared with petrodiesel fuel $[5,8,10,107,117-120]$.

An increase in nitrogen oxide (NOx) emissions was often reported [8,10,17,117,120-121], although no consensus has been determined. Some studies reported an NOx increase $[119,122]$, while others did not find significant differences between petrodiesel and biodiesel fuels [123], and yet others observed decreases when using biodiesel $[114,124]$. Some causes of this disagreement are the large variety of engine technologies, operating conditions, biodiesel fuels, and measurement techniques used $[10,117]$. It is generally accepted that NOx emissions are influenced by coupled physical and 
chemical phenomena $[35,121,125]$. A mitigation of the effect of biodiesel on NOx emissions could be achieved by delaying injection (which is slightly advanced with biodiesel because of its physical properties) in combination with increasing exhaust-gas recirculation $[10,126]$, or by increasing the spray-cone angle combined with an advanced start of injection [127] or even by the use of indirect injection combustion systems (ICS) [128]. A reduction of the aromatic content and the use of cetane improvers were also proposed [10] and [125]. It is generally agreed that the formation of NOx during combustion is mainly controlled by the ignition delay of the fuel and the relative amounts of heat released during the premixed combustion phase and diffusion-controlled combustion phase [121]. A longer ignition delay strongly increases NOx formation [129]. According to the correlation between ignition characteristics and molecular structures of fuel components, increasing the fatty acid chain length, saturation, and chain length of the alcohol moiety are expected to decrease the ID, and therefore NOx formation $[105,129,130]$. Fortunately, sophisticated after-treatment systems required to achieve the 2010 diesel engine emission standards do not appear to be significantly affected by the use of biodiesel [125].

Table 3. Average trends from the reviewed literature regarding emissions and engine performance when using biodiesel from various raw materials. The comparative fuel behavior is described qualitatively via patterns: + when better, - when worse, $\approx$ when equivalent, $+/-$ when dependent of the engine operating conditions, and n/a when no information is available on the basis of the reviewed literature; the reason of this notation is also given ( $\uparrow$ for increase and $\downarrow$ for decrease, $\rightarrow$ corresponding implicitly to $\approx)$. Dependence on the nature of the lipid raw materials is specified in parenthesis by yes, no, or n/a (no information available in the reviewed literature).

\begin{tabular}{|c|c|c|c|}
\hline \multirow[t]{2}{*}{ Emissions } & FAME versus Petrodiesel & FAEE versus petrodiesel & FAME versus FAEE \\
\hline & \multicolumn{3}{|c|}{ (dependence in terms of the lipid raw materials) } \\
\hline \multicolumn{4}{|l|}{ Regulated } \\
\hline $\mathrm{NO}_{x}$ & $-\uparrow$ or $\approx$ (yes) & $+\downarrow /-\uparrow($ yes) & $-\uparrow(n o)$ \\
\hline PM & $+\downarrow$ (no) & $+\downarrow /-\uparrow($ no) & $+\downarrow /-\uparrow($ no) \\
\hline THC (or UHC) & $+\downarrow$ (no) & $+\downarrow$ (no) & $-\uparrow($ no) \\
\hline $\mathrm{CO}$ & $+\downarrow($ no) & $+\downarrow($ no) & $-\uparrow(n o)$ \\
\hline \multicolumn{4}{|l|}{ Non-regulated } \\
\hline PSD & $+\downarrow$ (yes) & $\mathrm{n} / \mathrm{a}$ & $\mathrm{n} / \mathrm{a}$ \\
\hline Large particles & $+\downarrow$ or $\approx($ yes $)$ & $\mathrm{n} / \mathrm{a}$ & $\mathrm{n} / \mathrm{a}$ \\
\hline Small particles & $+\uparrow$ or $\approx$ (yes) & $\mathrm{n} / \mathrm{a}$ & $\mathrm{n} / \mathrm{a}$ \\
\hline Ultrafine particles & $-\uparrow$ or $\approx$ (yes) & $\mathrm{n} / \mathrm{a}$ & $\mathrm{n} / \mathrm{a}$ \\
\hline Carbonyl compounds & $-\uparrow$ or $\approx$ (yes) & $\mathrm{n} / \mathrm{a}$ & $\mathrm{n} / \mathrm{a}$ \\
\hline PAH & $+\downarrow /-\uparrow$ (yes) & $\mathrm{n} / \mathrm{a}$ & $\mathrm{n} / \mathrm{a}$ \\
\hline $\mathrm{SO}_{x}$ & $+\downarrow$ (no) & $\mathrm{n} / \mathrm{a}$ & $\mathrm{n} / \mathrm{a}$ \\
\hline $\begin{array}{l}\mathrm{CO}_{2} \\
\text { Performance }\end{array}$ & $+\downarrow$ (no) & $+\downarrow /-\uparrow($ no) & $+\downarrow$ (no) \\
\hline Effective power (full road) & $-\downarrow(n / a)$ & $+\uparrow /-\downarrow(n / a)$ & $+\uparrow$ or $\approx(\mathrm{n} / \mathrm{a})$ \\
\hline BSFC & $-\uparrow(n o)$ & $+\downarrow /-\uparrow$ (yes) & $+\downarrow$ or $\approx(\mathrm{n} / \mathrm{a})$ \\
\hline BTE & $+\uparrow /-\downarrow$ or $\approx$ (yes) & $\approx$ (yes) & $+\uparrow$ or $\approx(\mathrm{n} / \mathrm{a})$ \\
\hline
\end{tabular}

Three other classes of harmful emissions remain subject to controversy: (i) the proportion of fine particles (less than $10 \mu \mathrm{m}$ ) emitted; (ii) polycyclic aromatic hydrocarbons (PAH); and (iii) carbonyl compounds, such as aldehydes and ketones, which are carcinogenic and mutagenic for the lighter ones and potential ozone-precursors. Most studies have reported decreases in the mean diameter of the particle size distributions (PSD) with biodiesel, attributed to a sharp decrease in the number of large particles by some authors, while others observed an increase in the number of smallest particles [131-133]. While reduction in soot emissions has been virtually always obtained when biodiesel was added to diesel fuel [133] and [134], no conclusive trend was observed regarding the emission of PAH: while RME and SME produced similarly low emissions of PAH compared to petroleum fuels, the opposite effect was observed with neat rapeseed oil [108]. However, Karavalakis et al. [113] observed that the addition of biodiesel led to an important increase in lowmolecular-weight PAH (phenanthrene and anthracene), and to an increase or reduction of heavier 
PAH species, depending on the nature of FAME used. This lack of knowledge is even greater regarding the emissions of carbonyl compounds, such as aldehydes and ketones, for which very few studies have been performed. Systematic increases in carbonyl emissions were detected by He et al. [135] with an engine fueled with SME compared to petrodiesel. However, Fontaras et al. [115] observed that low-concentration biodiesel blends had a minor impact on carbonyl compound emissions. Nevertheless, this impact was determined to be dependent on the nature of the biodiesel: some such as RME resulted in significant increases, while others such as palm oil FAME led to decreases. Hence, similar to the NOx issue, all harmful emissions are interdependent of the physical and chemical combustion parameters. Therefore, the impact of each should be investigated separately within a referential well defined framework allowing meaningful comparisons $[10,115,117,135]$.

Regarding engine performance, conventional engines can be operated with biodiesel without major modification. In addition, blended or neat biodiesel does not cause any loss of power output unless maximum power is demanded and generally leads to a similar thermal efficiency as petrodiesel fuel $[5,8,10,117,119]$. Nevertheless, an increase in fuel consumption because of the lower heating value of biodiesel compared to petrodiesel (Table 2) was reported [10]. Among the various tests of biodiesel blends, B20 ( $20 \%$ vol. FAME with $80 \%$ vol. petrodiesel) provided the maximum improvement in terms of emissions and performance and was recommended for long-term engine operation [8].

\subsubsection{FAEE biodiesel versus petrodiesel and analysis in terms of various biolipid raw materials}

Far fewer studies detail the effect of ethanol-derived biodiesel (FAEE) on diesel engine performance and emissions than the effect of methanol-derived biodiesel (FAME).

Peterson et al. [112] tested exhaust emissions from a diesel vehicle fueled with neat rapeseed oil ethyl esters (REE). The authors reported that UHC, CO and NOx emissions decreased by $55.6 \%$, $50.6 \%$, and $11.8 \%$, respectively, compared with petrodiesel fuel. An increase in $\mathrm{CO}_{2}(1.1 \%)$ and $\mathrm{PM}$ $(10.3 \%)$ was observed. Nevertheless, a blend of $20 \%$ REE and $80 \%$ petrodiesel led to a decrease in PM emission of $5.7 \%$ compared to neat petrodiesel fuel. Nearly the same trends were observed by Makareviciene and Janulis [136] with a direct injection 4 cylinders diesel Audi 80 engine fueled with petrodiesel, blends of biodiesel/petrodiesel (25-75\%vol.) or neat biodiesel. Substituting petrodiesel with neat REE, led to a reduction in $\mathrm{CO}$ and UHC emissions and smoke density of 7.2, 53.0, and $72.6 \%$, respectively, and a slight increase in NOx emissions (8.3\%). Nevertheless, the reverse trend was obtained for NOx emissions with the B25-B50 blends in REE/petrodiesel.

Al-Widyan et al. [137] studied the use of different blends of waste vegetable oil ethyl esters (WVOEE) with petrodiesel in a single-cylinder DI diesel engine. The blends produced fewer CO and UHC emissions than did neat petrodiesel. The authors also indicated that the blends burned more efficiently with less fuel consumption and higher power output. Overall, the best engine performances were obtained with 100\% WVOEE and 75:25 WVOEE/petrodiesel blends, while the 50:50 blend produced the lowest emissions. Puhana et al. [138] extended the latter work by separately testing petrodiesel and non-edible Mahua oil ethyl esters (MOEE). The authors reported that the fuel consumption for MOEE was higher than for petrodiesel fuel due to the combined effect of a low heating value and high density of MOEE. The emissions of $\mathrm{CO}, \mathrm{UHC}$, and NOx, were reduced by approximately 58,63 , and $12 \%$, respectively, in MOEE compared to petrodiesel fuel.

\subsubsection{FAME versus FAEE as biodiesel and analysis in terms of various biolipid raw materials}

In addition to the evaluation of REE performance versus petrodiesel, Makareviciene and Janulis [136] also compared the environmental effect of REE with RME. Results indicated that REE had better environmental effects than RME in terms of $\mathrm{CO}$, NOx, and smoke emissions. Moreover, REE was 
more biodegradable in an aqueous environment than RME. Lapuerta et al. [123] studied the performance characteristics and emissions of waste cooking oil methyl and ethyl esters (WCOME and WCOEE, respectively) in a 4 cylinder, turbocharged, direct injection Nissan diesel engine. The biodiesels were tested neat and blended at $30 \%$ and $70 \% \mathrm{vol}$. with a low-sulfur petrodiesel fuel. Exhaust analysis showed that WCOEE produced fewer NOx emissions than WCOME, explained by a lower premixed combustion of WCOEE. Total hydrocarbon emissions were also impacted by the type of alcohol used in the biodiesel production: emissions were increased with more volatile alcohol, meaning that WCOME was less favorable than WCOEE. Nevertheless, a slightly higher reduction in opacity and PM emissions was observed with WCOME (consistent with a higher oxygen content). Baiju et al. [122] performed a comparative evaluation of compression-ignition engine characteristics using methyl and ethyl esters of Karanja oil (KOME and KOEE, respectively), a non-edible oil that can be extensively grown in the wastelands of India. Compared with KOME, KOEE showed slightly higher viscosity and reduced cold-flow properties as well as a higher flash point. These observations, consistent with previous studies [106,139], make FAEE a safer fuel for storage and transport than FAME. Regarding engine performance and exhaust emissions, KOME yielded slightly better characteristics than KOEE in terms of power output, brake thermal efficiency (BTE), and fuel consumption. A slightly cleaner behavior for KOME was observed; however, these last results are in disagreement with other studies $[123,136]$ regarding $\mathrm{CO}$ and NOx emissions.

In summary, although biodiesel engine performance has been determined to be slightly inferior but similar overall to that of petrodiesel, biodiesel contributes to reduce pollutant emissions. Nevertheless, this argument must be confirmed, particularly for the non-regulated emissions and performance of FAEE versus FAME, with consideration of the lipid raw material origins. Modeling studies reinforced with suitable experimental information would provide a better understanding and more accurate prediction of engine performance and emissions for a given biodiesel fuel.

\section{Biodiesel combustion kinetics}

The oxidation kinetics of hydrocarbons has been the subject of numerous studies, as presented in recent literature reviews related to this subject $[140,141]$. However, fewer kinetic studies related to the oxidation of biodiesel have been performed at the experimental and modeling levels. The main reason for this lack of information is the molecular structure of actual biodiesel components (saturated and unsaturated fatty acid methyl or ethyl esters), which involves large aliphatic main chains of $14-20$ carbon atoms with $\mathrm{CH} 2-\mathrm{CH}=\mathrm{CH}$ and ester groups (Fig. 1, Table 1); this complexity in the molecular structure of reactants poses a significant challenge to modeling and experimental kinetic studies.

Therefore, this part of the review focuses on oxidation modeling of biodiesel (surrogates and neat fuel) using detailed chemical kinetic mechanisms and the experiments that are a prerequisite for the development and validation of the corresponding models. These models are generated using theoretical-based approaches that account for thermodynamic and kinetic phenomena.

\subsection{Main features of model development and validation}

\subsubsection{Detailed chemical kinetic models and their foundation}

Models based on detailed chemical kinetic mechanisms can accurately predict hydrocarbon reactivity in the presence of oxygen $[140,141]$. This success is mainly due to their core methodology, describing at the molecular level the chemical changes occurring during the reactions. This molecular approach has two results. First, most of the proposed mechanisms have been detailed systematically by using similar reaction classes and drawing a well-accepted general kinetic scheme related to the primary oxidation reactions of the fuel molecule and its derived species (molecular and radical species). The 
slight differences between the reaction classes used by the different research teams are described exhaustively in the Battin-Leclerc literature review [141] for the combustion of alkanes. Second, fuels comprising heavy molecules (with more than 6 carbon atoms) involve complex mechanisms. Therefore, two types of approaches were adopted in the development of mechanisms and derived kinetic models, depending on whether a computer was used or not. Mechanisms (and derived kinetic models), developed without computer assistance, were generally built iteratively by modules, starting with small esters and progressing to larger ones. For example, the research team of LLNL (Lawrence Livermore National Laboratory) developed successively models for methyl decanoate [142], methyl decenoates [143], then methyl stearate and oleate [144], and eventually a mixture representing the main components of soybean and rapeseed methyl esters [145]. The research team of Milan proposed lumped mechanisms (i.e. with globalization of species and/or elementary reactions) based on the same reaction classes for methyl butanoate [146], decanoate [147], and biodiesel fuels [148]. However, software-generated mechanisms did not include previous submechanisms and were usually smaller due to tailoring the required mechanisms to the operating conditions of study. Hence, with EXGAS software (which is primarily intended for hydrocarbons, such as alkanes [149,150] and alkenes [151] and extended to biodiesels and derivatives [152,153], as will be discussed in the following sections), only the significant classes of reactions for the temperature range of the study may be activated using a menu.

For all mechanisms, the thermodynamic properties were determined using software, i.e. THERM [154] for non-computer-based mechanisms and THERGAS [155] for computer-aided mechanisms. When no thermodynamic properties were available in the literature or stored in the software databank, the thermodynamic properties were automatically computed using the implemented method, usually the Benson method [156] with updated group contribution and bond additivity values. Concerning the kinetic properties, information available in the literature and stored in the software databank was used as a first option. When unavailable, the kinetic properties were usually estimated using correlations based on quantitative structure-reactivity relationships or quantum calculations. More precisely, each elementary reaction of the mechanism was written as a reversible reaction, and the reverse rate constant was computed from the corresponding forward rate constant and appropriate equilibrium constant $\mathrm{K}_{\mathrm{c}}=\mathrm{k}_{\text {forward }} / \mathrm{k}_{\text {reverse }}$ calculated from thermochemical data. Concerning EXGAS software, KINGAS subroutine [157] was devoted to kinetic property calculations.

\subsubsection{Model validation: experiments and environment modelling}

Oxidation experiments were conducted in various physical environments. The equipment used were selected to cover a wide range of reactors with different geometries (shock tube, premixed flames, diffusion flames, continuous jet-stirred reactor, plug-flow reactor, variable pressure-flow reactor, or internal combustion engines) in which experiments could be conducted at a wide range of temperatures, pressures, and biodiesel/oxygen equivalence ratios $\phi: \phi<1$ for fuel-lean mixtures and $\phi>1$ for fuel-rich mixtures (see nomenclature for definition). ${ }^{1}$ Most experiments provided speciesdependent and time-dependent information, which is particularly valuable and relevant for model development and validation (with the exception of shock tubes yielding integrated information, such as ignition delay). Therefore, experimental information generated from this large panel of physical environments warranted wide-ranging kinetic modeling. However, while kinetic modeling aims to

\footnotetext{
1 This definition corresponds to the traditional equivalence ratio, in which the oxygen atoms contained in a fuel molecule are still part of the fuel, functioning as an oxidizer during the oxidation process. Therefore, the concept of the oxygen equivalence ratio, which is considered a more appropriate representation of the mixture stoichiometry for oxygenated fuels than the traditional equivalence ratio, was introduced [1]. The oxygen equivalence ratio is defined as the oxygen content available in the reactant mixture divided by the amount of oxygen required for stoichiometric combustion.
} 
account for chemical phenomena, suitable and reliable reactor modeling is required to account for the physical environment. All of these features must be gathered to perform reliable predictions of the fuel behavior during ignition, combustion, and emission in diesel and homogeneous-charge compression-ignition (HCCl) engines. Within this context, CHEMKIN library software [158], which enables the modeling of a wide range of reactors, was the most widely used tool in the scientific community related to kinetics. The standardization of the CHEMKIN input data format for describing the reactions, rate parameters, thermodynamic data, and transport properties of species favored this common use by facilitating the exchange of models between scientists [141].

\subsubsection{Main guidelines of past research and the present review}

Two major paths were followed in past research related to biodiesel oxidation for the reliable prediction of engine behavior. The first path is devoted to oxidation experiments and kinetic modeling oriented to small methyl and ethyl esters with 1-4 carbon atoms $\left(C_{1}-C_{4}\right)$ in their aliphatic main chain. These works provided detailed information on the special features of methyl and ethyl ester group reactivity for future studies. The second path is devoted to generating experimental information by conducting the oxidation of actual biodiesel fuel and components and then comparing the experimental results with the simulation results obtained from the oxidation mechanisms of large $n$-alkanes. This approach assumes that large $n$-alkanes and fatty acid methyl or ethyl esters with the same number of carbon atoms in their aliphatic main chain behave similarly during oxidation.

Nevertheless, the authors agreed that all alkyl esters with an aliphatic main chain at $C_{1}-C_{2}$ were too small to be considered as potential biodiesel surrogates (i.e. methyl and ethyl formate or acetate [159-163]). The first molecule considered as an acceptable biodiesel surrogate among the small alkyl esters investigated was methyl butanoate with an aliphatic main chain at position $\mathrm{C}_{4}$. Studies that followed however showed that this molecule was not a very suitable surrogate fuel for biodiesel study in general, but rather a model providing insight into kinetics of the methyl ester function. Thus, further research extended the kinetic modeling capabilities to alkyl esters with larger aliphatic main chains for the reliable reproduction of the reactivity of actual biodiesel components, such as early (low-temperature) $\mathrm{CO}_{2}$ production from the ester group and burning similar to petrodiesel. These features are described in more detail in the following sections after the presentation of some additional chemical bases of the combustion process.

In the following, alkyl (methyl or ethyl) esters with aliphatic main chains comprised of $\mathrm{n}$ carbon atoms will be designated $C_{n}$-alkyl esters; thus $C_{n}$-methyl (ethyl) esters will designate molecules with $n$ $+1(n+2)$ carbon atoms. The experimental data generated for the oxidation (and occasionally pyrolysis) of alkyl esters selected as biodiesel surrogates are summarized in Table 4 [1,153,164-194], whereas the main features related to the developed chemical kinetic models are presented in Table 5 [142-148,151-155,157,166,167,169-171,174,182,184,188-190,197,201,203,206-216].

Table 4. Summary of the main experimental data generated for oxidation of alkyl esters selected as biodiesel surrogates.

\begin{tabular}{|c|c|c|c|c|c|}
\hline Fuel & $\begin{array}{c}\text { Equipment (Data } \\
\text { type) }\end{array}$ & Temperature $^{\mathrm{a}} / \mathrm{K}$ & Pressure/atm & $\begin{array}{c}\text { Equivalence ratio } \\
\varphi \text { (Fuel molar } \\
\text { fraction \%) }\end{array}$ & Reference \\
\hline \multirow[t]{6}{*}{$\begin{array}{c}\text { Methyl } \\
\text { Butanoate }\end{array}$} & FR (SP) & $500-900$ & 12.5 & $\begin{array}{c}0.35-1.5 \\
(800 \mathrm{ppm})\end{array}$ & [164] \\
\hline & CFNPF (SP) & - & 1 & $\begin{array}{c}- \\
\left(5000 \mathrm{ppm}+\mathrm{CH}_{4}\right)\end{array}$ & [165] \\
\hline & JSR (SP) & $800-1350$ & 1 & $1.13(0.075)$ & [166] \\
\hline & OFDF (SP) & - & 1 & $-(4.7)$ & \\
\hline & VPFR (SP) & $500-900$ & 12.5 & $0.35-1.5(-)$ & \\
\hline & ST (ID) & $1100-1670$ & $1-4$ & $0.25-1.5(-)$ & {$[167]$} \\
\hline
\end{tabular}




\begin{tabular}{|c|c|c|c|c|c|}
\hline & JSR (SP) & $850-1350$ & 1 & $1.0(0.075)$ & [168] \\
\hline & OFDF (SP) & $355-413$ & 1 & $-(4.7)$ & \\
\hline & JSR (SP) & $850-1400$ & 1 & $\begin{array}{c}0.375 \text { and } 0.75 \\
(0.075)\end{array}$ & [169] \\
\hline & $\mathrm{RCM}(\mathrm{ID})$ & $640-949$ & $10-40$ & $\begin{array}{c}0.33-1.0(1.59- \\
3.13)\end{array}$ & [170] \\
\hline & ST (ID) & $1250-1760$ & $1-4$ & $\begin{array}{c}0.25-1.5(1.0- \\
1.5)\end{array}$ & \\
\hline & $\mathrm{RCM}(\mathrm{ID})$ & $935-1117$ & $4.7-19.6$ & $\begin{array}{c}0.3-0.4(0.95- \\
1.27)\end{array}$ & [171] \\
\hline & $\mathrm{RCM}$ (ID) & $650-850$ & $3.9-19.7$ & $1.0(-)$ & {$[172]$} \\
\hline & ST ( $\mathrm{CO}_{2}$ yields) & $1260-1653$ & $1.4-1.7$ & $\begin{array}{l}\text { Pyrolysis study } \\
\qquad(2-3)\end{array}$ & [173] \\
\hline & JSR (SP) & $800-850$ & 1 & $0.5-1.0(2)$ & {$[174]$} \\
\hline & ST (ID) & $1250-2000$ & $7.6-9.1$ & $0.25-2(0.5-1)$ & \\
\hline & ST (ID) & $1060-1632$ & $1.2-11.4$ & $\begin{array}{c}0.5-2.0(0.64- \\
3.32)\end{array}$ & [175] \\
\hline & $\begin{array}{c}\mathrm{ST}(\mathrm{ID}, \\
\text { concentration } \\
\text { time-histories of } \\
\mathrm{CO}, \mathrm{CO}_{2}, \mathrm{CH}_{3} \text {, and } \\
\left.\mathrm{C}_{2} \mathrm{H}_{4}\right)\end{array}$ & $1200-1800$ & 1.5 & $\begin{array}{c}\text { Pyrolysis }(0.01 \\
0.05,0.1)\end{array}$ & [176] \\
\hline & $\begin{array}{l}\text { Counterflow } \\
\text { flame (Laminar } \\
\text { flame speeds and } \\
\text { local extinction } \\
\text { strain rates) }\end{array}$ & $403^{\underline{b}}$ & 1 & $0.7-1.6$ (in air) & [177] \\
\hline & $\begin{array}{l}\text { Counterflow } \\
\text { flame (extinction } \\
\text { strain rates) }\end{array}$ & $298 / 500$ & 1 & $-(0.10-0.18)$ & [178] \\
\hline & $\begin{array}{l}\text { Flame and bomb } \\
\text { (Laminar flame } \\
\text { speeds and local } \\
\text { extinction strain } \\
\text { rates) }\end{array}$ & $353^{\underline{b}}$ & $1.0-3.0$ & $0.7-1.7$ (in air) & [179] \\
\hline & Flame (SP) & - & 0.04 (30 Torr) & $1.56(0.14)$ & [180] \\
\hline \multirow[t]{4}{*}{ Methyl Crotonate } & OFDF (SP) & $355-413$ & 1 & $-(4.7)$ & [168] \\
\hline & JSR (SP) & $850-1350$ & 1 & $1(0.075)$ & \\
\hline & JSR (SP) & $850-1400$ & 1 & $\begin{array}{c}0.375 \text { and } 0.75 \\
(0.075)\end{array}$ & [169] \\
\hline & Flame (SP) & - & 0.04 (30 Torr) & $1.56(0.14)$ & [181] \\
\hline Methyl & JSR (SP) & $500-1100$ & 10 & $0.5,1,1.5(0.1)$ & [182] \\
\hline Hexanoate & $\mathrm{RCM}$ (ID) & $650-850$ & 3.9-19.7 & $1.0(-)$ & [172] \\
\hline Methyl & JSR (SP) & $550-1150$ & 10 & $1-2(0.001)$ & [183] \\
\hline \multicolumn{6}{|l|}{ Heptanoate } \\
\hline Methyl & JSR (SP) & $800-1350$ & 1 & $0.5-2(-)$ & [184] \\
\hline \multirow[t]{2}{*}{ Octanoate } & OFDF (SP) & $400-420$ & 1 & $-(1.8 \%)$ & \\
\hline & ST (ID) & $1250-1550$ & $1-10$ & $\begin{array}{c}0.5,1,2(380- \\
1380 \mathrm{ppm})\end{array}$ & [185] \\
\hline \multirow[t]{6}{*}{$\begin{array}{c}\text { Methyl } \\
\text { Decanoate }\end{array}$} & $\begin{array}{c}\text { Variable CR } \\
\text { octane rating } \\
\text { (HRR versus CAD } \\
\text { and exhaust SP) }\end{array}$ & $400-2000$ & $\begin{array}{c}\text { CR range } 4.0- \\
13.7\end{array}$ & $0.25-2$ & {$[186,187]$} \\
\hline & $\begin{array}{l}\text { Flame (Counter } \\
\text { flow extinction } \\
\text { and ignition } \\
\text { critical } \\
\text { conditions) }\end{array}$ & $900-1600$ & 1 & $0.5-1.5(-)$ & [188] \\
\hline & OFDF (SP) & $400-420$ & 1 & $-(1.8 \%)$ & [189] \\
\hline & JSR (SP) & $500-1100$ & 1.06 & $1(0.0021)$ & [153] \\
\hline & JSR (SP) & $773-1123$ & 1.05 & $\begin{array}{l}\text { Pyrolysis study } \\
(0.0218)\end{array}$ & [190] \\
\hline & Counterflow & $403^{\underline{b}}$ & 1 & $0.7-1.6$ (in air) & {$[177]$} \\
\hline
\end{tabular}




\begin{tabular}{|c|c|c|c|c|c|}
\hline & $\begin{array}{l}\text { flame (Laminar } \\
\text { flame speeds and } \\
\text { local extinction } \\
\text { strain rates) }\end{array}$ & & & & \\
\hline & Flow Reactor (SP) & $873-1123$ & 1.7 & $\begin{array}{c}\text { Pyrolysis (0.091- } \\
0.625)\end{array}$ & [191] \\
\hline & ST (ID) & 653-1336 & $15-16$ & $\begin{array}{l}0.5,1.0,1.5 \text { (in } \\
\text { air) }\end{array}$ & [192] \\
\hline & ST (ID) & $838-1381$ & $1.71-8.63$ & $0.1-2$ (in air) & [193] \\
\hline $\begin{array}{c}\text { Methyl Palmitate } \\
\text { (MP) ( } n- \\
\text { decane/MP) }\end{array}$ & JSR (SP) & $500-1000$ & 1 & $1(0.002)$ & [194] \\
\hline $\begin{array}{l}\text { Methyl Oleate } \\
\text { (MO) ( } n- \\
\text { decane/MO) }\end{array}$ & JSR (SP) & $550-1100$ & 1 & $1(0.002)$ & [195] \\
\hline $\begin{array}{l}\text { Methyl Oleate } \\
\text { (neat) }\end{array}$ & ST (ID) & $1100-1400$ & $3.5-7.0$ & $0.6-2.4(0.04)$ & [196] \\
\hline Methyl Linoleate & ST (ID) & $1100-1400$ & $3.5-7.0$ & $0.6-2.4(0.04)$ & [196] \\
\hline \multirow[t]{2}{*}{$\begin{array}{l}\text { Rapeseed Methyl } \\
\text { Esters (RME) }\end{array}$} & JSR (SP) & $800-1400$ & $1-10$ & $\begin{array}{c}0.25,0.5,1,1.5 \\
(0.05)\end{array}$ & [197] \\
\hline & Tube (SP) & $823-1073$ & 1 & Pyrolysis (0.07) & [198] \\
\hline $\begin{array}{l}\text { Palm Methyl } \\
\text { Esters }\end{array}$ & $\begin{array}{l}\text { Flame (Laminar } \\
\text { flame speeds) }\end{array}$ & $470^{\underline{b}}$ & 1 & $0.7-1.4$ (in air) & [199] \\
\hline $\begin{array}{l}\text { RME/kerosene } \\
\text { (20/80 mol/mol) }\end{array}$ & JSR (SP) & $740-1200$ & 1 & $0.5-1.5(-)$ & [200] \\
\hline $\begin{array}{c}\text { Commercial B30 } \\
\text { fuel \& B30 } \\
\text { surrogate fuel } \\
\text { (49\% } n \text {-decane, } \\
21 \% 1- \\
\text { methylnaphtlene, } \\
30 \% \text { MO in mole) }\end{array}$ & JSR (SP) & $560-1030$ & 6 and 10 & $\begin{array}{c}0.25-1.5 \\
(10,300 \text { ppm of } \\
\text { carbon) }\end{array}$ & [201] \\
\hline $\begin{array}{l}\text { Ethyl Propanoate } \\
\text { (EP) with MiBu, } \\
\text { PrAc, and iPrAc } \\
\text { for comparison }\end{array}$ & CFNPF (SP) & - & 1 & $\begin{array}{c}-(5000 \\
\left.\mathrm{ppm}+\mathrm{CH}_{4}\right)\end{array}$ & [165] \\
\hline \multirow[t]{3}{*}{ EP } & ST (ID) & $1140-1675$ & $1-4$ & $0.25-1.5(-)$ & [167] \\
\hline & JSR (SP) & $750-1100$ & 1 & $0.3-1(0.1)$ & [202] \\
\hline & RCM (ID) & $935-1117$ & $4.7-19.6$ & $\begin{array}{c}0.3-0.4(0.95- \\
1.27)\end{array}$ & [171] \\
\hline \multirow[t]{2}{*}{ Ethyl Butanoate } & ST (ID) & $1250-2000$ & $7.6-9.1$ & $0.25-2(0.417)$ & [174] \\
\hline & Laminar PFR & $500-1200$ & $1.03-1.35$ & $0.5-1.6(0.013)$ & [203] \\
\hline \multirow[t]{2}{*}{ Ethyl Pentanoate } & JSR (SP) & $560-1160$ & 10 & $\begin{array}{c}06,1,2 \\
(1000 \mathrm{ppm})\end{array}$ & [204] \\
\hline & $\begin{array}{l}\text { Bomb (Laminar } \\
\text { flame speeds) }\end{array}$ & $423^{\underline{b}}$ & $1-10$ & $0.7-1.4$ (in air) & \\
\hline $\begin{array}{l}\text { EN with: MN, } \\
\text { MN2EN, and } \\
\text { MN3EN for } \\
\text { comparison }\end{array}$ & $\begin{array}{c}\text { CFR motored } \\
\text { engine (exhaust } \\
\text { SP) }\end{array}$ & $\begin{array}{l}\text { 650-1850 (bulk } \\
\text { cylinder gas } \\
\text { temperature) }\end{array}$ & CR range $4.43-15$ & $0.25(-)$ & {$[1]$} \\
\hline $\begin{array}{l}\text { EHX with } \mathrm{MH} \text { for } \\
\text { comparison }\end{array}$ & $\begin{array}{c}\text { CFR motored } \\
\text { engine (exhaust } \\
\text { SP) }\end{array}$ & $\begin{array}{l}\text { 600-1650 (bulk } \\
\text { cylinder gas } \\
\text { temperature) }\end{array}$ & $\begin{array}{c}\text { CR range } 4.43- \\
10.5\end{array}$ & $0.25(-)$ & [205] \\
\hline
\end{tabular}


Table 5. Summary of the main features related to the chemical kinetic models proposed for the oxidation of alkyl esters selected as biodiesel surrogates.

\begin{tabular}{|c|c|c|c|c|c|c|c|c|c|c|c|}
\hline \multirow[t]{2}{*}{ Fuel $^{a}$} & \multicolumn{2}{|c|}{ Model features } & \multicolumn{4}{|c|}{ Software features } & \multicolumn{4}{|c|}{ Model applicability conditions } & \multirow[t]{2}{*}{ Ref. } \\
\hline & $\begin{array}{l}\mathrm{Nb} \text {. of } \\
\text { reactions }\end{array}$ & $\begin{array}{l}\mathrm{Nb} \text {. of } \\
\text { species }\end{array}$ & $\begin{array}{l}\text { Thermoch } \\
\text { emical } \\
\text { property } \\
\text { estimatio } \\
n\end{array}$ & $\begin{array}{l}\text { Kinetic } \\
\text { rate } \\
\text { estimati } \\
\text { on }^{\mathrm{b}}\end{array}$ & $\begin{array}{c}\text { Mechanis } \\
\mathrm{m} \\
\text { generator } \\
\mathrm{c}\end{array}$ & $\begin{array}{l}\text { CHEMK } \\
\text { IN } \\
\text { modul } \\
\text { e used }\end{array}$ & $\begin{array}{l}\text { Low } \\
T \\
(\approx 50 \\
0 \mathrm{~K})\end{array}$ & $\begin{array}{l}\text { High } \\
T \\
(\approx 130 \\
0 \mathrm{~K})\end{array}$ & $\begin{array}{l}\text { Low } P \\
(\approx 1 \text { at } \\
\mathrm{m})\end{array}$ & $\begin{array}{c}\text { Mod } \\
\text { erate } \\
P \\
(\approx 10 \\
\text { atm })\end{array}$ & \\
\hline \multirow[t]{7}{*}{$\begin{array}{l}\text { Methyl } \\
\text { Butanoate }\end{array}$} & 1219 & 264 & $\begin{array}{l}\text { THERM } \\
{[154],} \\
{[206,207]^{d}}\end{array}$ & N.C.H. & None & SENKIN & $\checkmark$ & - & $\checkmark$ & - & [208] \\
\hline & 1498 & 295 & $\begin{array}{l}\text { THERM } \\
{[154],} \\
{[206,209]}\end{array}$ & N.C.H. & None & N.D. ${ }^{e}$ & $\checkmark$ & $\checkmark$ & $\checkmark$ & $\checkmark$ & [166] \\
\hline & N.D. ${ }^{f}$ & N.D. ${ }^{f}$ & $\begin{array}{l}\text { THERM } \\
{[154],} \\
{[206,209]}\end{array}$ & N.C.H. & None & $\begin{array}{l}\text { HCT } \\
{[210]^{\mathrm{g}}}\end{array}$ & - & $\checkmark$ & $\checkmark$ & - & [167] \\
\hline & 1545 & 275 & $\begin{array}{l}\text { THERM } \\
{[154],} \\
{[206,209]}\end{array}$ & N.C.H. & None & $\begin{array}{l}\mathrm{HCT} \\
{[210]^{\mathrm{g}}}\end{array}$ & $\checkmark$ & $\checkmark$ & $\checkmark$ & $\checkmark$ & [170] \\
\hline & [167] & & $\begin{array}{l}\text { THERM } \\
\text { [154] }\end{array}$ & N.C.H. & None & $\begin{array}{l}\mathrm{HCT} \\
{[210]^{\mathrm{g}}}\end{array}$ & $\checkmark$ & & & $\begin{array}{l}\checkmark \\
(\approx 20 \\
\text { atm) }\end{array}$ & [171] \\
\hline & 1317 & 203 & $\begin{array}{l}\text { THERGAS } \\
\text { [155] }\end{array}$ & $\begin{array}{l}\text { KINGAS } \\
\text { [157] }\end{array}$ & $\begin{array}{l}\text { EXGAS } \\
{[151]}\end{array}$ & $\begin{array}{l}\text { SENKIN } \\
\text { PSR }\end{array}$ & $\checkmark$ & $\checkmark$ & $\checkmark$ & $\checkmark$ & [174] \\
\hline & 46 & $\approx 25$ & ND & N.C.H. & None & $\begin{array}{l}\text { PSR } \\
\text { SENKIN } \\
\text { PREMI } \\
X\end{array}$ & - & $\checkmark$ & $\checkmark$ & $\checkmark$ & [146] \\
\hline $\begin{array}{l}\text { Methyl } \\
\text { Crotonate }\end{array}$ & 1516 & 301 & $\begin{array}{l}\text { THERGAS } \\
\text { [155] }\end{array}$ & N.C.H. & None & $\begin{array}{l}\text { PSR } \\
\text { OPPDIF } \\
\text { F }\end{array}$ & $\checkmark$ & $\checkmark$ & $\checkmark$ & - & [169] \\
\hline \multirow[t]{2}{*}{$\begin{array}{l}\text { Methyl } \\
\text { Hexanoate }\end{array}$} & 1875 & 435 & $\begin{array}{l}{[208]+\text { TH }} \\
\text { ERGAS } \\
{[155]}\end{array}$ & N.C.H. & None & PSR & $\checkmark$ & $\checkmark$ & $\checkmark$ & $\checkmark$ & [182] \\
\hline & 2440 & 401 & $\begin{array}{l}\text { THERGAS } \\
\text { [155] }\end{array}$ & $\begin{array}{l}\text { KINGAS } \\
\text { [157] }\end{array}$ & $\begin{array}{l}\text { EXGAS } \\
{[152,153]}\end{array}$ & PSR & $\checkmark$ & $\begin{array}{l}J \\
(1100 \\
\mathrm{K})\end{array}$ & - & $\checkmark$ & [153] \\
\hline $\begin{array}{l}\text { Methyl } \\
\text { Heptanoate }\end{array}$ & 3236 & 531 & $\begin{array}{l}\text { THERGAS } \\
\text { [155] }\end{array}$ & $\begin{array}{l}\text { KINGAS } \\
\text { [157] }\end{array}$ & $\begin{array}{l}\text { EXGAS } \\
{[152,153]}\end{array}$ & PSR & $\checkmark$ & $\begin{array}{l}J \\
(1100 \\
\mathrm{K})\end{array}$ & - & $\checkmark$ & [153] \\
\hline $\begin{array}{l}\text { Methyl } \\
\text { Octanoate }\end{array}$ & 2781 & 383 & $\begin{array}{l}\text { MHX } \\
{[182]}\end{array}$ & N.C.H. & None & $\begin{array}{l}\text { PSR } \\
\text { OPPDIF } \\
\text { F }\end{array}$ & - & $\checkmark$ & $\checkmark$ & - & [184] \\
\hline \multirow[t]{2}{*}{$\begin{array}{l}\text { Methyl } \\
\text { Decanoate }\end{array}$} & 8820 & 3012 & $\begin{array}{l}\text { THERM } \\
{[154]}\end{array}$ & N.C.H. & None & $\begin{array}{l}\text { ICE }^{\mathrm{h}} \\
\text { PSR } \\
\text { AUROR } \\
\text { A }\end{array}$ & $\checkmark$ & $\checkmark$ & $\checkmark$ & $\checkmark$ & [142] \\
\hline & 713 & 125 & $\begin{array}{l}\text { Skeletal me } \\
\text { Herbinet et }\end{array}$ & $\begin{array}{l}\text { hanism de } \\
\text { l. model }\end{array}$ & $\begin{array}{l}\text { red from } \\
\text { 2] }\end{array}$ & $\begin{array}{l}\text { Flame } \\
\text { Master }\end{array}$ & $\checkmark$ & $\checkmark$ & $\checkmark$ & - & [188] \\
\hline \multirow[t]{6}{*}{ NO prod! } & $\begin{array}{l}8820+10 \\
5\end{array}$ & $3012+20$ & $\begin{array}{l}\text { Mechanism } \\
\text { [142] and Sr }\end{array}$ & $\begin{array}{l}\text { from Herbi } \\
\text { ith et al. }\end{array}$ & $\begin{array}{l}\text { tet et al. } \\
1]\end{array}$ & $\begin{array}{l}\mathrm{HCPR}^{\mathrm{I}} \\
\mathrm{HCCl}^{\mathrm{K}}\end{array}$ & $\checkmark$ & $\checkmark$ & - & $\checkmark$ & [212] \\
\hline & 2992 & 648 & $\begin{array}{l}\text { Skeletal me } \\
\text { Seshadri et }\end{array}$ & $\begin{array}{l}\text { hanism de } \\
\text { l. model [1 }\end{array}$ & 8] from & $\begin{array}{l}\text { OPPDIF } \\
\mathrm{F}\end{array}$ & $\checkmark$ & $\checkmark$ & - & $\checkmark$ & [189] \\
\hline & 3231 & 324 & $\begin{array}{l}\text { THERGAS } \\
{[155]}\end{array}$ & $\begin{array}{l}\text { KINGAS } \\
\text { [157] }\end{array}$ & $\begin{array}{l}\text { EXGAS } \\
{[152,153]}\end{array}$ & PSR & - & $\begin{array}{l}\text { Pyrol } \\
\text { ysis } \\
\checkmark\end{array}$ & $\begin{array}{l}\text { Study } \\
\checkmark\end{array}$ & - & [190] \\
\hline & 7775 & 1247 & $\begin{array}{l}\text { THERGAS } \\
{[155]}\end{array}$ & $\begin{array}{l}\text { KINGAS } \\
{[157]}\end{array}$ & $\begin{array}{l}\text { EXGAS } \\
{[152,153]}\end{array}$ & PSR & $\checkmark$ & - & $\checkmark$ & - & [213] \\
\hline & $\approx 10000$ & $\approx 350$ & N.D. & N.C.H. & None & $\begin{array}{l}\text { PSR } \\
\text { SENKIN } \\
\text { PREMI } \\
X\end{array}$ & $\checkmark$ & $\checkmark$ & $\checkmark$ & $\checkmark$ & [147] \\
\hline & 2276 & 7086 & $\begin{array}{l}\text { THERM } \\
{[154]}\end{array}$ & N.C.H. & None & $\begin{array}{l}\text { PSR } \\
\text { SENKIN } \\
\text { PREMI } \\
X\end{array}$ & $\checkmark$ & $\checkmark$ & $\checkmark$ & $\checkmark$ & [214] \\
\hline MD5EN & N.D. ${ }^{f}$ & N.D..$^{f}$ & $\begin{array}{l}\text { THERM } \\
{[154]^{\prime}}\end{array}$ & N.C.H. & None & $\begin{array}{l}\text { PSR } \\
\text { SENKIN }\end{array}$ & $\checkmark$ & $\checkmark$ & $\checkmark$ & $\checkmark$ & [143] \\
\hline MD9EN & N.D. ${ }^{f}$ & N.D. ${ }^{f}$ & THERM & N.C.H. & None & PSR & $\checkmark$ & $\checkmark$ & $\checkmark$ & $\checkmark$ & [143] \\
\hline
\end{tabular}




\begin{tabular}{|c|c|c|c|c|c|c|c|c|c|c|c|}
\hline & & & {$[154]^{1}$} & & & SENKIN & & & & & \\
\hline nC16 (RME) & 1841 & 225 & $\begin{array}{l}\text { THERGAS } \\
{[155]}\end{array}$ & N.C.H. & None & PSR & $\checkmark$ & $\checkmark$ & $\checkmark$ & $\checkmark$ & $\begin{array}{l}{[197,} \\
215]\end{array}$ \\
\hline $\begin{array}{l}\mathrm{MB}+n C 7+ \\
\mathrm{PME}(\mathrm{RME})\end{array}$ & 1472 & 309 & $\begin{array}{l}\text { THERM } \\
{[154]}\end{array}$ & N.C.H. & None & SENKIN & - & $\checkmark$ & - & $\checkmark$ & [216] \\
\hline $\begin{array}{l}\text { B30 } \\
\text { surrogate } \\
\text { fuel }(49 \% n \text { - } \\
\text { decane, } \\
21 \% 1- \\
\text { methylnaph } \\
\text { tlene, } 30 \% \\
\text { MO in } \\
\text { mole) }\end{array}$ & 7748 & 1964 & $\begin{array}{l}\text { THERGAS } \\
\text { [155] and } \\
\text { literature } \\
\text { data [201] }\end{array}$ & N.C.H. & None & PSR & $\checkmark$ & $\begin{array}{l}(1030 \\
\mathrm{K})\end{array}$ & $\checkmark$ & $\checkmark$ & [201 \\
\hline MDO & 13,004 & 2012 & $\begin{array}{l}\text { THERGAS } \\
{[155]}\end{array}$ & $\begin{array}{l}\text { KINGAS } \\
{[157]}\end{array}$ & $\begin{array}{l}\text { EXGAS } \\
{[152,153]}\end{array}$ & PSR & $\checkmark$ & - & $\checkmark$ & - & [213] \\
\hline MM & 20,412 & 3061 & $\begin{array}{l}\text { THERGAS } \\
{[155]}\end{array}$ & $\begin{array}{l}\text { KINGAS } \\
{[157]}\end{array}$ & $\begin{array}{l}\text { EXGAS } \\
{[152,153]}\end{array}$ & PSR & $\checkmark$ & - & $\checkmark$ & - & [213] \\
\hline MP & 30,425 & 4442 & $\begin{array}{l}\text { THERGAS } \\
{[155]}\end{array}$ & $\begin{array}{l}\text { KINGAS } \\
{[157]}\end{array}$ & $\begin{array}{l}\text { EXGAS } \\
{[152,153]}\end{array}$ & PSR & $\checkmark$ & - & $\checkmark$ & - & [213] \\
\hline MST and & $>17,000$ & $>3500$ & \multicolumn{4}{|c|}{ Extended work by Herbinet et al. $[142,143]$} & $\checkmark$ & $\checkmark$ & - & $\checkmark$ & [144] \\
\hline MO & & & $\begin{array}{l}\text { THERM } \\
{[154]}\end{array}$ & N.C.H. & None & PSR & & & & & \\
\hline MST & 43,444 & 6203 & $\begin{array}{l}\text { THERGAS } \\
{[155]}\end{array}$ & $\begin{array}{l}\text { KINGAS } \\
{[157]}\end{array}$ & $\begin{array}{l}\text { EXGAS } \\
{[152,153]}\end{array}$ & PSR & $\checkmark$ & - & $\checkmark$ & - & [213] \\
\hline MP, MST, & 20,000 & $>4800$ & \multicolumn{4}{|c|}{ Extended work by Herbinet et al. $[142,143]$} & $\checkmark$ & $\checkmark$ & $\checkmark$ & $\checkmark$ & [145] \\
\hline $\begin{array}{l}\text { MO, MLO, } \\
\text { MLN }\end{array}$ & & & $\begin{array}{l}\text { THERM } \\
\text { [154] }\end{array}$ & N.C.H. & None & PSR & & & & & \\
\hline $\begin{array}{l}\text { MP, MST, } \\
\text { MO, MLO, } \\
\text { MLN }\end{array}$ & $\approx 13,000$ & $\approx 420$ & N.D. & N.C.H. & None & $\begin{array}{l}\text { SENKIN } \\
\text { PSR } \\
\text { SENKIN }\end{array}$ & $\checkmark$ & $\checkmark$ & $\checkmark$ & $\checkmark$ & [148] \\
\hline \multirow[t]{2}{*}{ EP } & 786 & 139 & $\begin{array}{l}\text { THERM } \\
{[154]}\end{array}$ & N.C.H. & None & $\begin{array}{l}\mathrm{HCT} \\
{[210]^{\mathrm{g}}}\end{array}$ & - & $\checkmark$ & $\checkmark$ & - & [167] \\
\hline & N.D. ${ }^{f}$ & N.D. ${ }^{f}$ & $\begin{array}{l}\text { THERM } \\
{[154]}\end{array}$ & N.C.H. & None & $\begin{array}{l}\text { HCT } \\
{[210]^{\mathrm{g}}}\end{array}$ & $\checkmark$ & $\checkmark$ & $\checkmark$ & $\checkmark$ & $\begin{array}{l}{[167,} \\
171]\end{array}$ \\
\hline \multirow[t]{2}{*}{ EB } & 1101 & 115 & $\begin{array}{l}\text { THERGAS } \\
{[155]}\end{array}$ & $\begin{array}{l}\text { KINGAS } \\
{[157]}\end{array}$ & $\begin{array}{l}\text { EXGAS } \\
{[152,153]}\end{array}$ & SENKIN & - & - & $\checkmark$ & $\checkmark$ & $\begin{array}{l}{[174,} \\
203]\end{array}$ \\
\hline & 1035 & 117 & & & & PSR & $\checkmark$ & $\checkmark$ & $\checkmark$ & - & \\
\hline
\end{tabular}

${ }^{a}$ See nomenclature for species names.

${ }^{b}$ N.C.H.: non-computer-helped method: the rate constants for the reverse reactions $k_{\text {reverse }}$ were computed from the forward rate constants $k_{\text {forward }}$ and the equilibrium constants $K_{c}$ calculated using the appropriate thermochemical data according to $\mathrm{K}_{\mathrm{c}}=\mathrm{k}_{\text {forward }} / \mathrm{k}_{\text {reverse }}$.

'None: no mechanism generator was used for developing the kinetic models which were instead built hierarchically.

dUpdated information related to group values by Fisher et al. [208] was published later by Curran et al. [207].

e N.D.: No details mentioned.

${ }^{f}$ Model derived from Fisher et al. proposal [208] with some changes, but resulting number of reactions and species involved are not mentioned in the manuscript.

${ }^{\mathrm{g}} \mathrm{HCT}$ was used instead of CHEMKIN.

${ }^{\mathrm{h}}$ Internal combustion engine model (single zone). iNO formation (GRI-Mech 3.0 mechanism).

${ }^{\mathrm{j}}$ Homogeneous constant-pressure reactor model.

${ }^{k}$ Homogeneous-charge compression-ignition model.

'Herbinet et al. [143] also took the opportunity to mention openly that the activation energy for abstraction of secondary $\mathrm{H}$-atoms by $\mathrm{HO}$ radicals needed to be corrected from $-3500 \mathrm{cal} \mathrm{mol}^{-1}$ (as used in the initial version of MD oxidation model) to $-35 \mathrm{cal} \mathrm{mol}^{-1}$.

\subsection{General kinetic scheme and classes of elementary reactions in the combustion process}

The general kinetic scheme and classes of elementary reactions used in the development of the detailed chemical kinetic mechanisms proposed in the literature for the oxidation of esters with aliphatic main chains in $C_{n}, n \geq 4$, are similar to those established by Curran et al. for the oxidation of $\mathrm{n}$-heptane and iso-octane $[217,207]$. Extension to alkyl-ester molecules was achieved by including the chemical information specific to the ester moiety. The resulting kinetic scheme is shown in Fig. 4 [142], whereas the classes of elementary reactions considered for the generation of alkyl-ester 
oxidation mechanisms are listed in Table 6 . Note that the main changes to these elementary reactions in relation to the work by Curran et al. [207,217] are in the class of reaction 26, which is specific to esters [167], and in the chemical nature of the species hidden behind the notation $R, R^{\prime}, Q$, and $\mathrm{X}$. The naming conventions adopted in Fig. 4 and Table 6 are as follows: $\mathrm{R}$ and $\mathrm{R}^{\prime}$ to denote alkyl or alkyl-ester radicals or structures and $Q$ and $X$ are used to denote $C_{n} H_{2 n}$ or $C_{n} H_{2 n} C O O$ species and structures.

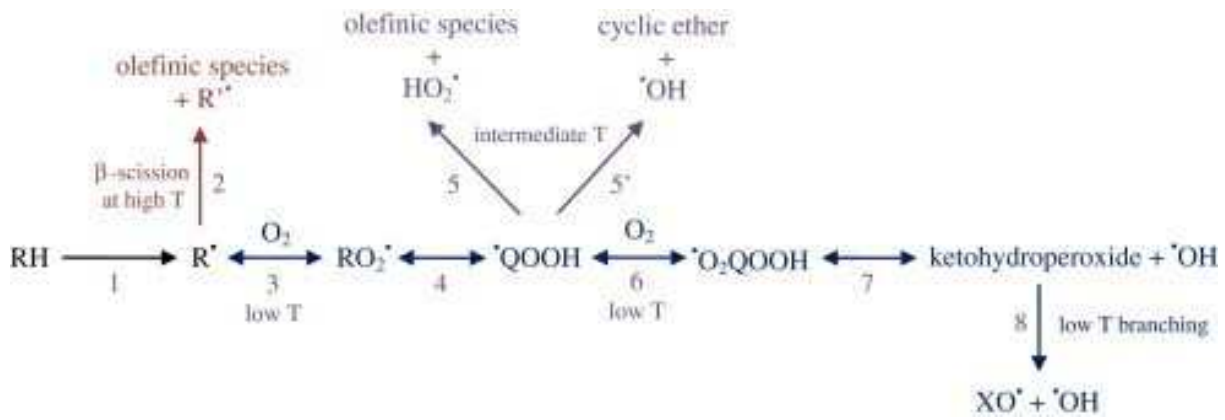

Figure 4. Primary oxidation reaction pathways common to alkyl esters (aliphatic main chain $C_{n}, n \geq 4$ ) [142] - Adaptation from Curran et al. work [217]. The labeled reactions (1-8) are further described in the main text.

Table 6. Major classes of elementary reactions used as basement by oxidation mechanisms proposed in the literature (extension of the work by Curran et al. [207] and [217] to alkyl-ester oxidation).

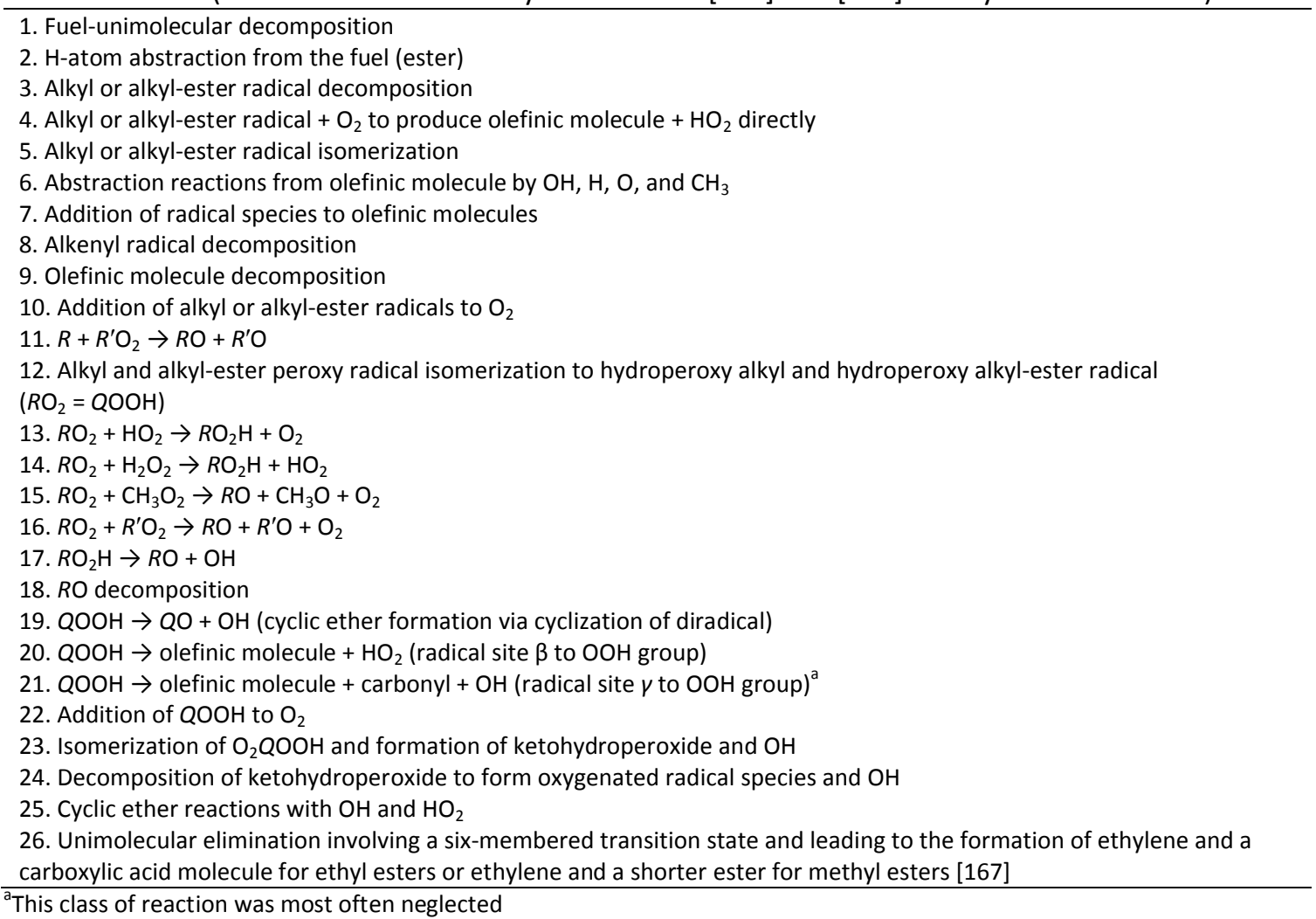

As shown in Fig. 4, two main reaction pathways with a three-way branch on one pathway clearly stand out depending on the temperature range. First, the oxidation of the ester $\mathrm{RH}$ is initiated by the abstraction of a hydrogen atom ( $\mathrm{H}$-abstraction or metathesis) from $\mathrm{RH}$ (at low and high temperatures) and the unimolecular decomposition of $\mathrm{RH}$ (at high temperatures only), which leads to the formation of alkyl and alkyl-ester radicals $\mathrm{R}$ (reaction 1, Fig. 4). These radicals may follow 
either the high-temperature- or low-temperature-dominant pathway. At high temperatures (above $800 \mathrm{~K}$ ), unimolecular decomposition by $\beta$-scission is the dominant route and yields olefins or olefinic esters (stable molecules) as well as smaller radical species R' (reaction 2, Fig. 4) with the contribution of isomerization reactions. Olefins and olefinic esters formed through these primary routes react through the same types of reactions as the fuel molecules and through other reactions specifically because of the presence of the double bond, such as the radical-mediated addition to the double bond and decomposition by retro-ene reactions leading to two smaller 1-alkenes through a sixmembered ring transition state [142]. At low temperatures (approximately 500-600 K), the alkyl and alkyl-ester radicals $\mathrm{R}$ mainly undergo $\mathrm{O}_{2}$ addition, leading to alkyl and alkyl-ester peroxy radicals $\mathrm{RO}_{2}$ (reaction 3, Fig. 4), which react by isomerization to hydroperoxy alkyl and hydroperoxy alkyl-ester radicals $\mathrm{QOOH}$ (reaction 4, Fig. 4). At this point of the oxidation scheme, a three-way branch allows $\mathrm{QOOH}$ radicals to undergo three pathways, the first two pathways of which dominate at intermediate temperatures: a $\mathrm{C}-\mathrm{O} \beta$-scission decomposition to olefinic alkyl or alkyl-ester stable molecule plus $\mathrm{HO}_{2}$ (reaction 5, Fig. 4); a decomposition to cyclic ether plus $\mathrm{OH}$ (reaction 5', Fig. 4); or a second addition to $\mathrm{O}_{2}$ leading to hydroperoxy peroxy radicals $\mathrm{O}_{2} \mathrm{QOOH}$ (reaction 6, Fig. 4). The product of this second addition leads to $\mathrm{OH}$ and ketohydroperoxide species (reaction 7, Fig. 4), which undergo low-temperature branching reactions by decomposition into two radicals (reaction 8, Fig. 4) [141] and [142]. Thus, it can be clearly observed that at two points of the general oxidation scheme, intermediate radicals ( $\mathrm{R}$ formed by reaction 1 and $\mathrm{QOOH}$ formed by reaction 4, Fig. 4) are involved in competitive reaction pathways that dominate at different temperature ranges and lead to chain reactions of different modes. Globally, these competitive reaction pathways belong to two classes: (i) unimolecular decomposition by $\beta$-scission occurring at high and intermediate temperature, which leads to chain-propagation reactions that produce one radical flux (reactions 2,5 , or $5^{\prime}$ in Fig. 4), and (ii) addition to $\mathrm{O}_{2}$ occurring at lower temperatures, which leads to chain-branching reactions that produce two radical flux (reactions 3 and 6 leading to reaction 8 in Fig. 4). This competition between branching channels and propagation channels may induce global variation in reactivity over some temperature ranges, as outlined in the following section.

\subsection{Negative temperature coefficient and related low-temperature phenomena}

The negative temperature coefficient (NTC) region signifies a zone of temperature in which the overall reaction rate decreases with temperature (Fig. 5). This phenomenon is specific to lowtemperature oxidation processes and occurs usually around 500-800 K. Some explanations can be directly deduced from the general oxidation scheme (Fig. 4). At low temperatures, chain-branching occurs mainly via the $\mathrm{O}_{2}$ addition reaction pathway, going through a ketohydroperoxide species (reaction 7, Fig. 4). As the temperature increases, the chain-propagation reactions of the $\mathrm{QOOH}$ species (reactions 5 and 5 ', Fig. 4) increase because the energy barrier for their formation is easily overcome, which leads to the formation of cyclic ether species and olefinic molecules at the expense of the reaction pathways through the ketohydroperoxide species (reaction 8 , Fig. 4). The increasing importance of these propagation channels leads to a lower system reactivity, which is observed as the NTC region. However, propagation channels related to high-temperature $\beta$-scissions should also partially contribute to these phenomena. Thus, NTC phenomena could be considered as a chemical transient regime that allows for continuously transitioning from established low- to hightemperature chemical regimes. This feature is commonly encountered in hydrocarbon combustion [207] and [217] and is observed when operating at engine conditions with esters comprised of aliphatic main chains in $C_{n}, n \geq 5$ [172], where oxidation mechanisms may involve temperature rangedependent competition between chain-propagating channels and chain-branching channels. 


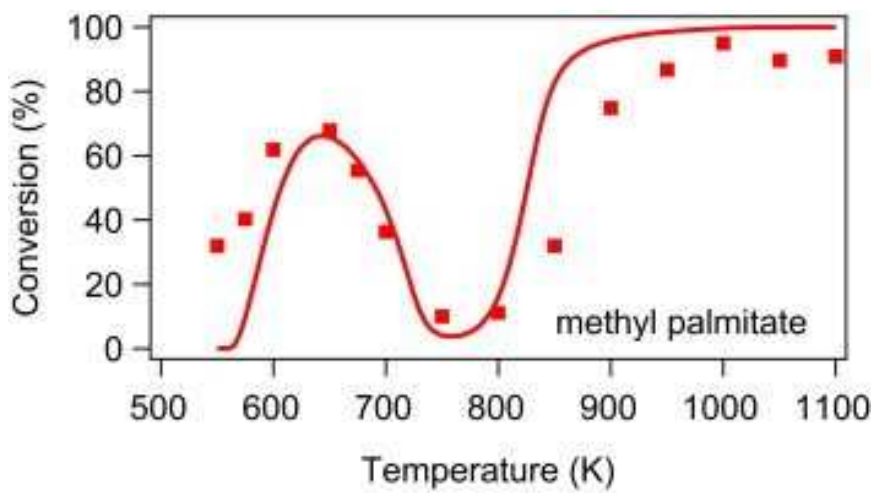

Figure 5. Illustration of the negative temperature coefficient region: experimental and computed conversions of methyl palmitate in a JSR as a function of temperature [143].

\subsection{Methyl ester kinetic studies}

Kinetic studies related to methyl esters are presented first for methyl butanoate (molecule firstly selected in the literature as biodiesel surrogate and thereafter considered as a mimetic molecule of the methyl ester function) and the corresponding ester with a double bond in the middle of the aliphatic main chain, i.e. methyl crotonate (methyl (E)-2-butenoate). Next, studies are presented for molecules tending toward suitable biodiesel surrogates (methyl hexanoate, methyl octanoate, methyl decanoate, methyl-5- and methyl-9-decenoate), then for actual biodiesel molecules (methyl myristate, methyl palmitate, methyl stearate, methyl oleate, methyl linoleate, and methyl linolenate), and ultimately for two commercial biodiesels (rapeseed and soybean oil methyl esters).

\subsubsection{Oxidation of methyl butanoate as mimetic molecule of the methyl ester function}

As might be expected, methyl butanoate $(\mathrm{MB})\left(\mathrm{C}_{3} \mathrm{H}_{7}(\mathrm{CO}) \mathrm{OCH}_{3}\right)$ has been the subject of many published kinetic studies ([146,164-172,174,175,177-180,208], Tables 4 and 5 with regard to oxidation kinetics studies). This $\mathrm{C}_{4}$-methyl ester was originally selected as the mimetic model molecule of larger biodiesel components $\left(C_{16}-\right.$ to $C_{22}$-methyl esters, Table 1$)$ to limit the number of possible products formed to a manageable level and to produce a reaction mechanism of a manageable size that can focus on the kinetic description of the methyl ester moiety. The main chemical structure features that account for the combustion characteristics of biodiesel, i.e. the ester moiety and aliphatic main chain of sufficient length, were thought to be conserved; thus, MB seemed large enough to allow the fast $\mathrm{RO}_{2}$ isomerization reaction (class of reactions 12 in Table 6; reaction 4 in Fig. 4) that play a key role in low-temperature chemistry and control fuel autoignition under conditions found in diesel engines.

The first detailed chemical kinetic mechanism for the combustion of MB was developed by Fisher et al. [208]. This proposal comprised 264 species and 1219 reactions (Table 5) and was established by introducing the submechanism that described the specificity of $M B$ oxidation to the existing oxidation mechanism for $n$-heptane by Curran et al. [217]. The model by Fisher et al. [208] was tested against the limited combustion data available at the time. These tests were conducted in closed vessels under low temperatures and subatmospheric conditions [218-221]. SENKIN software [222] was used to perform the chemical kinetic calculations in which the thermodynamic properties of the methyl esters and their decomposition products were estimated using Benson group and bond additivity methods [156] with updated values [206,207]. Some qualitative agreement was obtained; however, calculations consistently indicated higher overall reactivity by a factor of 10-50 compared with experiments. In addition, according to the same experimental data, the Fisher et al. model [208] predicted a weak NTC behavior for MB oxidation that the authors explained using their proposed 
low- and high-temperature reaction pathways (Fig. 6). Obviously, part of the general kinetic scheme presented in Fig. 4 for alkyl-ester oxidation can be observed when applied to the MB molecule as this pathway is directly derived from Fisher et al., who presented the original idea. In particular, competition between chain-propagation channels (from unimolecular decomposition at high temperatures) and chain-branching channels (from $\mathrm{O}_{2}$ addition at low temperatures), which usually involves NTC phenomena, can be observed at two points of the Fisher et al. model (Fig. 6).

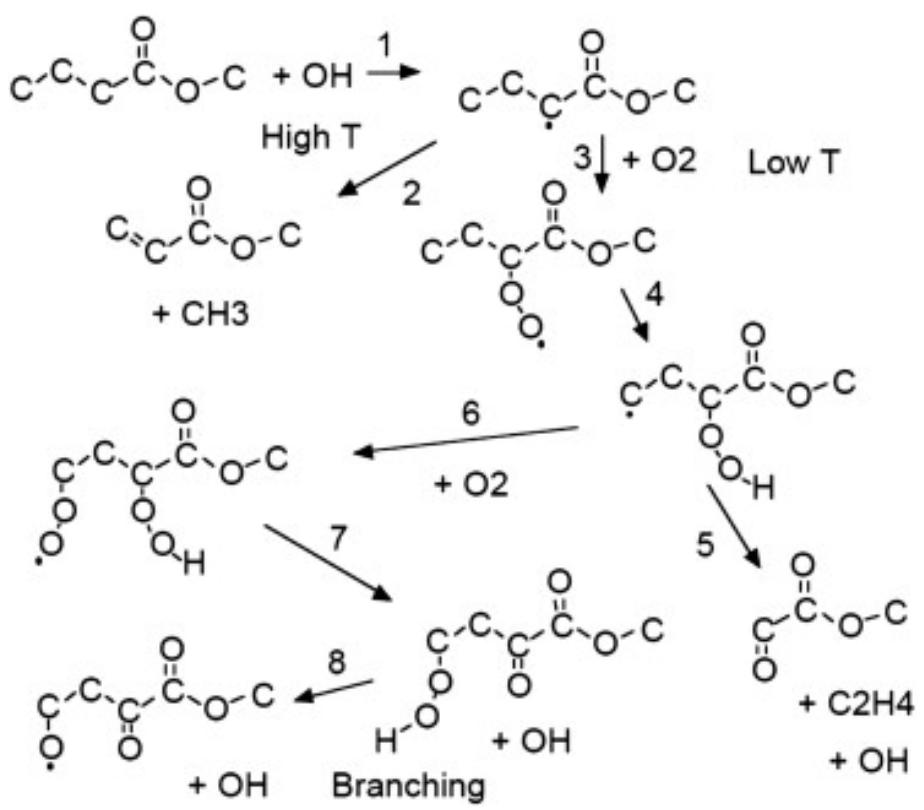

Figure 6. Key low- and high-temperature reaction pathways of methyl butanoate oxidation for one of the four possible hydrogen abstraction sites (Fisher et al. model [208]). The three other possible sites

of $\mathrm{H}$-abstraction are on the carbon in $\alpha$ or $\beta$ to the radical site illustrated in the figure and on the methyl group of the ester function.

Later on, Marchese et al. [164] conducted MB oxidation experiments in a flow reactor operating under moderate pressures over low to medium temperatures with various equivalence ratios and a residence time set to a constant value (Table 4). Profiles of the reactant, intermediates and product species determined during the experiments were compared with simulation results obtained from the Fisher et al. [208] mechanism. Good agreement was obtained under stoichiometric conditions; however, the model overpredicted the experimentally observed reactivity under fuel-rich conditions and under-predicted reactivity under fuel-lean conditions.

More recently, Gail et al. [166] proposed a revised version of the Fisher et al. mechanism [208], which was developed and validated based on the new experimental results they generated over a wide range of operating conditions using various pieces of equipment: a jet-stirred reactor (JSR), a Princeton variable pressure-flow reactor (VPFR), and an opposed-flow diffusion flame (OFDF) (Table 4). Molar fraction profiles for a large number of molecular species were obtained from JSR and VPFR experiments: $\mathrm{MB}$ and $\mathrm{O}_{2}$ (reactant); $\mathrm{H}_{2}, \mathrm{CO}, \mathrm{CO}_{2}$, acetylene, methane, ethylene, ethane, propene, propane, formaldehyde, methanol, methyl propenoate, propanal, and methyl crotonate (major products); allene, acrolein, acetaldehyde, 1-butene, and 1,3-butadiene (minor products); and methyl vinyl ketone (trace compounds). Similar trends were observed for the profiles of the molecular species obtained from OFDF experiments. The new model by Gail et al. [166] consisted of 295 species and 1498 reactions. Three rate constant parameters from the original mechanism by Fisher et al. [208] were modified as described in Table 7, and a C4-submechanism taken from Dagaut et al. [223] was added to simulate the measured 1-butene and 1,3-butadiene profiles. The kinetic modeling is in reasonable agreement with the experimental results. However, under JSR conditions, MB reactivity 
was under-predicted by the model at low temperatures. Concerning OFDF data, the same model generally showed higher reactivity for intermediate species and lower reactivity for $M B$ than observed in experimental studies. However, the model accurately reproduced the VPFR data at stoichiometric conditions, and neither the model nor experiments showed evidence of the NTC region. It was only for the leanest investigated equivalence ratios that little NTC behavior could be observed between 600 and $800 \mathrm{~K}$.

Table 7. Reaction rate constants modified by Gail et al. [166] (bold) in the Fisher et al. mechanism for $\mathrm{MB}[208]^{\mathrm{a}}$

\begin{tabular}{|c|c|c|c|}
\hline Reaction & $A$ & $n$ & $E_{a}$ \\
\hline $\mathrm{CH}_{3} \mathrm{CH}_{2} \mathrm{CH}_{2}(\mathrm{C}=\mathrm{O}) \mathrm{OCH}_{3}+\mathrm{H} .=\mathrm{H}_{2}+\mathrm{CH}_{3} \mathrm{CH}_{2} \cdot \mathrm{CH}(\mathrm{C}=\mathrm{O}) \mathrm{OCH}_{3}$ & $2.52 E+14$ & 0.00 & 7300 \\
\hline $\mathrm{CH}_{3} \mathrm{CH}_{2} \mathrm{CH}_{2}(\mathrm{C}=\mathrm{O}) \mathrm{OCH}_{3}+\mathrm{H} .=\mathrm{H}_{2}+\mathrm{CH}_{3} \mathrm{CH}_{2} \cdot \mathrm{CH}(\mathrm{C}=\mathrm{O}) \mathrm{OCH}_{3}$ & $1.00 \mathrm{E}+14$ & 0.00 & 7300 \\
\hline $\mathrm{CH}_{3} \mathrm{O} \cdot(+\mathrm{M})=\mathrm{CH}_{2} \mathrm{O}+\mathrm{H} \cdot(+\mathrm{M})$ & $5.45 \mathrm{E}+13$ & 0.00 & 13,500 \\
\hline $\mathrm{CH}_{3} \mathrm{O} \cdot(+\mathrm{M})=\mathrm{CH}_{2} \mathrm{O}+\mathrm{H} .(+\mathrm{M})$ & $1.38 \mathrm{E}+21$ & -6.65 & 33,190 \\
\hline $\mathrm{C}_{2} \mathrm{H}_{3} .+\mathrm{O}_{2}=\mathrm{CH}_{2} \mathrm{O}+\mathrm{HCO}$ & $1.70 E+29$ & -5.31 & 6500 \\
\hline $\mathrm{C}_{2} \mathrm{H}_{3} \cdot+\mathrm{O}_{2}=\mathrm{CH}_{2} \mathrm{O}+\mathrm{HCO}$. & $1.66 \mathrm{E}+13$ & -1.39 & 1013 \\
\hline
\end{tabular}

${ }^{\mathrm{a}}$ The rate coefficients are listed in the generalized Arrhenius form $\left(\mathrm{k}=\mathrm{AT} \mathrm{T}^{\mathrm{n}} \cdot \exp (-\mathrm{Ea} / \mathrm{RT})\right)$ where units are mol, $\mathrm{cm}, \mathrm{s}, \mathrm{cal}, \mathrm{and}$ K.

The same year, Metcalfe et al. [167] reported the oxidation of MB in a shock tube (ST). Ignition delay times were measured behind reflected-shock waves at two moderate pressures ( 1 and $4 \mathrm{~atm}$ ) over the temperature range of $1100-1670 \mathrm{~K}$ for various equivalence ratios (Table 4). Their experimental results showed that at both pressures, the ignition delay times increased as MB concentration increased. Furthermore, the detailed mechanism proposed by the authors for MB oxidation was derived from the Fisher et al. model [208], and some changes were made as mentioned in the following.

- The $\mathrm{H}_{2} / \mathrm{O}_{2}$ mixture submechanism has been replaced with the mechanism published by O'Conaire et al. [224].

- The bond enthalpy of the $\alpha \mathrm{C}-\mathrm{H}$ bond to a carbonyl group has been decreased to $93.6 \mathrm{kcal} \mathrm{mol}^{-1}$ from $96.2 \mathrm{kcal} \mathrm{mol}^{-1}$ because of quantum theory-based estimations by El-Nahas et al. [225]. Note that the value adopted by Metcalfe et al. [167] corresponds to the group additivity estimates and not to the ab initio calculations $\left(94.2 \mathrm{kcal} \mathrm{mol}^{-1}\right)$ made by El-Nahas et al. [225].

- The rate constants for MB radical decomposition have been adjusted to account for alkyl and alkoxyl radical decomposition according to the work by Curran [226].

- The high-pressure limit rate constant expressions for unimolecular ester fuel decomposition reactions have been decreased by $66 \%$ (multiplied by 0.33 ).

- Unimolecular fuel decomposition reactions have been treated using quantum Rice-RamspergerKassel theory to account for pressure falloff (variation of the rate constant as a function of pressure).

- A six-centered unimolecular elimination reaction that yields ethylene and methyl ethanoate (methyl acetate) was added (Fig. 7) with an activation energy of $68 \mathrm{kcal} \mathrm{mol}^{-1}$ [225]. However, the authors found that this last pathway (Fig. 7) contributed very little $(<1 \%)$ to MB decomposition for the conditions investigated during the work. 


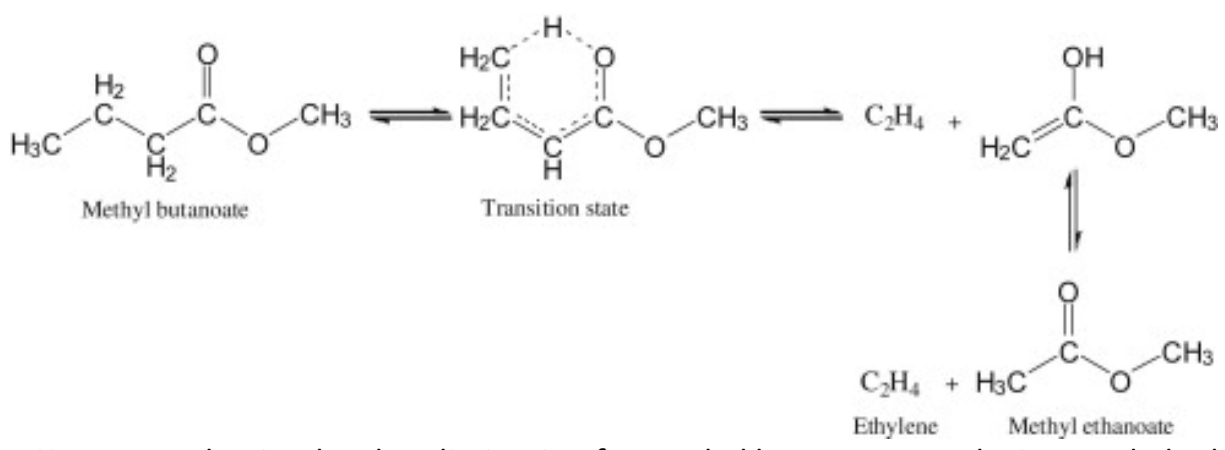

Figure 7. Six-centered unimolecular elimination for methyl butanoate producing methyl ethanoate and ethylene [167].

Following this work, measurements of ignition delay for MB were performed by Dooley et al. [170] at low temperatures (640-949 $\mathrm{K}$ ) in a rapid compression machine (RCM) and at higher temperatures $(1250-1760 \mathrm{~K})$ in a shock tube (Table 4). The autoignition of methyl butanoate was observed to follow the Arrhenius law $(\tau=A \cdot \exp (-E / R T))$ over all conditions studied. The authors also proposed a detailed chemical kinetic mechanism, which is an extensively modified version from their previous work [167]. The main changes are listed below (an exhaustive description is presented in Dooley et al. [170]):

- Some bond enthalpy values originally presented in Fisher et al. [208] were reevaluated using THERM [154] with updated $\mathrm{H} / \mathrm{C} / \mathrm{O}$ and bond dissociation groups $[206,209]$. These values concern the $\mathrm{nC}_{3} \mathrm{H}_{7} \mathrm{C}(\mathrm{O}) \mathrm{O}-\mathrm{CH}_{3}$ and $\mathrm{nC}_{3} \mathrm{H}_{7}-\mathrm{C}(\mathrm{O}) \mathrm{OCH}_{3}$ bonds reduced to 86.8 and $89.9 \mathrm{kcal} \mathrm{mol}^{-1}$ from 101.2 and $92.0 \mathrm{kcal} \mathrm{mol}^{-1}$, respectively. These values are in substantial agreement with the work by El-Nahas et al. [225], who proposed 87.0 and $93.4 \mathrm{kcal} \mathrm{mol}^{-1}$, respectively, using ab initio calculations.

- $\mathrm{A} \mathrm{C}_{4}$-submechanism (involving species with 4 carbon atoms) was added based on the work by Curran et al. [207,217] to account for $\mathrm{C}_{4}$ species observed in Gaïl et al. [166].

- The low-temperature chemistry describing the isomerization of alkylperoxyl radicals to hydroperoxyl alkyl radicals (reaction 4, Fig. 4) was not included. This choice was motivated by the experimentally non-observed low-temperature reactivity.

The new mechanism consisted of 275 species and 1545 reactions and was validated against the data generated by the authors (in RCM and ST) and those reported in the literature (in a flow reactor [164], a ST [167], a JSR and an OFDF [166]). The simulations were in good agreement with the experimental data selected. However, the agreement with the RCM ignition delays was less accurate. In addition, analysis of the kinetic model confirmed that different reaction pathways become prominent depending on the operating conditions, such as temperature, pressure, and equivalence ratio. In particular, the importance of unimolecular fuel decomposition at high temperatures (reaction 1, Fig. 4), the processes of the $\mathrm{HO}_{2}$ radical at low to intermediate temperatures (steps successive to reaction 5, Fig. 4), and the importance of fuel alkyl-radical decomposition (reaction 2 , Fig. 4) under fuel-rich conditions were highlighted. In addition, from their results in shock tubes and RCM, Dooley et al. [170] concluded that MB did not exhibit NTC behavior.

To meet the internal combustion engine conditions, Walton et al. [171] performed the experimental ignition of MB using an RCM over low temperatures (935-1117 K) and moderate pressures (4.7-19.6 atm) (Table 4). The rate constants of some MB reactions from the Metcalfe et al. mechanism [167] were slightly modified to improve the agreement between the computed and experimental results. These reactions are presented in Table 8. Overall, the agreement between the computed and experimental results was excellent for all temperatures. In addition, the results support the assumption that $\mathrm{MB}$ consumption would be dominated by relatively slow bimolecular $\mathrm{H}$-atom abstraction reactions. 
Table 8 . Reaction rate constants modified by Walton et al. [171] from Metcalfe et al. mechanism [167] related to MB oxidation. ${ }^{a}$

\begin{tabular}{|c|c|c|c|}
\hline Reaction & $A$ & $n$ & $E_{a}$ \\
\hline $\mathrm{CH}_{3} \mathrm{CH}_{2} \mathrm{CH}_{2}(\mathrm{C}=\mathrm{O}) \mathrm{OCH}_{3}+\mathrm{HO}_{2} .=\mathrm{H}_{2} \mathrm{O}_{2}+. \mathrm{CH}_{2} \mathrm{CH}_{2} \mathrm{CH}_{2}(\mathrm{C}=\mathrm{O}) \mathrm{OCH}_{3}$ & $1.90 \mathrm{E}+12$ & 0.00 & 20,440 \\
\hline $\mathrm{CH}_{3} \mathrm{CH}_{2} \mathrm{CH}_{2}(\mathrm{C}=\mathrm{O}) \mathrm{OCH}_{3}+\mathrm{HO}_{2} .=\mathrm{H}_{2} \mathrm{O}_{2}+\mathrm{CH}_{3} \cdot \mathrm{CHCH}_{2}(\mathrm{C}=\mathrm{O}) \mathrm{OCH}_{3}$ & $1.30 \mathrm{E}+12$ & 0.00 & 17,690 \\
\hline $\mathrm{CH}_{3} \mathrm{CH}_{2} \mathrm{CH}_{2}(\mathrm{C}=\mathrm{O}) \mathrm{OCH}_{3}+\mathrm{HO}_{2}=\mathrm{H}_{2} \mathrm{O}_{2}+\mathrm{CH}_{3} \mathrm{CH}_{2} \cdot \mathrm{CH}(\mathrm{C}=\mathrm{O}) \mathrm{OCH}_{3}$ & $1.30 \mathrm{E}+12$ & 0.00 & 17,690 \\
\hline $\mathrm{CH}_{3} \mathrm{CH}_{2} \mathrm{CH}_{2}(\mathrm{C}=\mathrm{O}) \mathrm{OCH}_{3}+\mathrm{HO}_{2} .=\mathrm{H}_{2} \mathrm{O}_{2}+\mathrm{CH}_{3} \mathrm{CH}_{2} \mathrm{CH}_{2}(\mathrm{C}=\mathrm{O}) \mathrm{O} . \mathrm{CH}_{2}$ & $1.90 \mathrm{E}+12$ & 0.00 & 20,440 \\
\hline $\mathrm{CH}_{3} \mathrm{CH}_{2} \mathrm{CH}_{2}(\mathrm{C}=\mathrm{O}) \mathrm{OCH}_{3}+\mathrm{H} .=\mathrm{H}_{2}+. \mathrm{CH}_{2} \mathrm{CH}_{2} \mathrm{CH}_{2}(\mathrm{C}=\mathrm{O}) \mathrm{OCH}_{3}$ & $1.88 \mathrm{E}+05$ & 2.75 & 6280 \\
\hline $\mathrm{CH}_{3} \mathrm{CH}_{2} \mathrm{CH}_{2}(\mathrm{C}=\mathrm{O}) \mathrm{OCH}_{3}+\mathrm{H} .=\mathrm{H}_{2}+\mathrm{CH}_{3} \cdot \mathrm{CHCH}_{2}(\mathrm{C}=\mathrm{O}) \mathrm{OCH}_{3}$ & $1.30 \mathrm{E}+06$ & 2.40 & 4471 \\
\hline $\mathrm{CH}_{3} \mathrm{CH}_{2} \mathrm{CH}_{2}(\mathrm{C}=\mathrm{O}) \mathrm{OCH}_{3}+\mathrm{H} .=\mathrm{H}_{2}+\mathrm{CH}_{3} \mathrm{CH}_{2} \cdot \mathrm{CH}(\mathrm{C}=\mathrm{O}) \mathrm{OCH}_{3}$ & $1.30 \mathrm{E}+06$ & 2.40 & 4471 \\
\hline $\mathrm{CH}_{3} \mathrm{CH}_{2} \mathrm{CH}_{2}(\mathrm{C}=\mathrm{O}) \mathrm{OCH}_{3}+\mathrm{H} .=\mathrm{H}_{2}+\mathrm{CH}_{3} \mathrm{CH}_{2} \mathrm{CH}_{2}(\mathrm{C}=\mathrm{O}) \mathrm{O} . \mathrm{CH}_{2}$ & $1.88 \mathrm{E}+05$ & 2.75 & 6280 \\
\hline
\end{tabular}

${ }^{2}$ The remainder of the mechanism from Metcalfe et al. [167] was unchanged. The rate coefficients are listed in the generalized Arrhenius form ( $\mathrm{k}=\mathrm{ATn} \cdot \exp (-\mathrm{Ea} / \mathrm{RT}))$ where units are $\mathrm{mol}, \mathrm{cm}, \mathrm{s}, \mathrm{cal}$, and $\mathrm{K}$.

Furthermore, Hakka et al. [174] performed an experimental and modeling study of the oxidation of $M B$ and ethyl butanoate (EB) in a ST and a JSR. From the JSR experiments (operating conditions: atmospheric pressure at two moderate temperatures $(800$ and $850 \mathrm{~K}$ ) for various residence times, Table 4), molar fraction profiles versus residence time could be generated for various molecular species: $\mathrm{MB}$ and $\mathrm{O}_{2}$, which relates the reactants; $\mathrm{CO}, \mathrm{CO}_{2}$, methane $\left(\mathrm{CH}_{4}\right)$, acetylene $\left(\mathrm{C}_{2} \mathrm{H}_{2}\right)$, ethylene $\left(\mathrm{C}_{2} \mathrm{H}_{4}\right)$, propane $\left(\mathrm{C}_{2} \mathrm{H}_{6}\right)$, methyl acrylate (methyl propenoate), and methyl crotonate (methyl (E)2-butenoate), which relates the intermediates and final products. Concerning the new experimental data generated in the ST, ignition delay times were performed by recording $\mathrm{OH}$ emissions behind reflected-shock waves for various operating conditions (at moderate pressure over a large range of high temperatures (1250-2000 K) as well as in fuel-rich, stoichiometric and fuel-lean compositions, Table 4). A new mechanism for MB was proposed using the first version of EXGAS software [149-151] and extended to account for short ester reactants [174]. In addition, for this class of compounds, the bond enthalpy of the $\mathrm{C}-\mathrm{H}$ bond adjacent to the carbonyl group was taken to be equal to $95.6 \mathrm{kcal} \mathrm{mol}^{-1}$, as proposed by Luo [227] for ethyl propanoate (EP). The mechanism of MB oxidation could be applied from low to high temperatures and involved 203 species and 1317 reactions (Table 5). The simulations were performed using SENKIN (for ST-related results) and PSR (for JSR-related results) modules of CHEMKIN software [158]. The authors reported that the agreement between the experimental and simulated ignition delays of MB was satisfactory. The same trend was observed with the ignition delay times previously measured by Metcalfe et al. [167] and Walton et al. [171]. Nevertheless, the kinetic model overpredicted MB consumption and production of $\mathrm{CO}$ and $\mathrm{CO}_{2}$ but under-predicted ethylene formation when compared to experimental results obtained in a JSR. However, the consumption of $\mathrm{O} 2$ and formation of $\mathrm{CH}_{4}$, methyl acrylate, and methyl crotonate were correctly reproduced. Reaction flux analysis performed under ST and JSR conditions (at 1370 and $800 \mathrm{~K}$, respectively, and for $50 \%$ conversion in $\mathrm{MB}$ ) showed that $\mathrm{MB}$ is mainly consumed by $\mathrm{H}$-abstraction with $\mathrm{H}, \mathrm{OH}$, and $\mathrm{HO}_{2}$ radicals, which are the main contributors.

Recently, to provide complementary data on MB autoignition, Akih-Kumgeh and Bergthorson [175] measured ignition delay times behind reflected-shock waves (by $\mathrm{CH}$ emissions) at moderate pressures (1.2-11.4 atm) and high temperatures (1060-1632 K) for various equivalence ratios in oxygen/argon mixtures (Table 4). Measurements were also performed for $n$-heptane as fuel (instead of $\mathrm{MB}$ ) for comparison. The generated experimental results for $\mathrm{MB}$ were compared with simulations obtained using the modified version of the Fisher et al. model [208] by Dooley et al. [170] along with the Hakka et al. model [174]. CANTERA software by Goodwin [228] was used to model the shock tube ignition process as a constant-volume adiabatic reactor. The authors determined that MB and $n$ heptane had comparable high-temperature ignition delay times under stoichiometric conditions; however, differences were observed under other conditions. In addition, good agreement was observed between the data generated by the authors and previously published data $[167,174]$, while the two test models deviated somewhat $[170,174]$. The authors highlighted that the model by Dooley et al. [170] generally predicted longer ignition delays than the model by Hakka et al. [174], and the models were in good agreement at low temperatures with more pronounced deviations at 
higher temperatures. In addition, the two test models deviated from experiments under rich conditions (while good agreement was obtained under stoichiometric conditions).

Yang et al. [180] studied the oxidation of three $\mathrm{C}_{5} \mathrm{H}_{10} \mathrm{O}_{2}$ ester isomers (MB, methyl iso-butanoate, and $\mathrm{EP}$ ) in premixed flames using photo-ionization mass spectrometry and monochromated synchrotron radiation. Significant differences in the compositions of key reaction intermediates were observed and explained by performing kinetic analyses of the detailed kinetic models developed in this study. Dooley et al. [178] measured extinction limits of $M B, n$-heptane, and $M B / n$-heptane diffusion flames with nitrogen dilution in counter flow with air. They observed that MB diffusion flames have a much lower extinction strain rate than $\mathrm{n}$-heptane diffusion flames and that the extinction strain rate of $\mathrm{n}$-heptane/MB diffusion flames increases significantly as the $\mathrm{n}$-heptane fraction is increased. The modeling study showed that the inhibiting effect of $M B$ is due to the difference in the energy contents and to formation of hydroperoxy radicals that interfere with the chain-branching reactions involving $\mathrm{H}$-atoms and $\mathrm{OH}$ radicals.

Grana et al. [147] developed a lumped kinetic model for the oxidation and thermal decomposition of MB. This lumped model also contains the chemistry for other small esters: methyl formate, methyl acrylate and methyl crotonate which are intermediates formed in the oxidation of MB. The model was successfully validated against a large set of experimental data obtained in a wide range of operating conditions $[164,166,170,171,174,177,179]$.

Thus, thanks to sustained efforts on the development of experiments and kinetic models for MB oxidation, rapid progress has been made in the understanding of the oxidation chemistry of the methyl ester function $\left(-\mathrm{COOCH}_{3}\right)$. Nevertheless, some refinements are still required in order to achieve a better agreement between the various prediction models and experimental results. An alternative would be to check the consistency both of the whole of the experiments and of the whole of the models, via close collaborations between research teams.

\subsubsection{Pyrolysis of methyl butanoate}

Oxidation mechanisms at high temperatures include characteristic reactions of pyrolysis as a purely thermal process (for example, fuel-unimolecular decompositions or bimolecular $\mathrm{H}$-abstractions from the fuel). Therefore, it is interesting to highlight some studies on ester pyrolysis, as they can be used as core partial sub-mechanisms to study the combustion of ester species.

Farooq et al. [173] studied the high-temperature thermal decomposition of three methyl esters (methyl acetate, methyl propionate, and $\mathrm{MB}$ ) in a ST by measuring the $\mathrm{CO}_{2}$ mole fraction timehistories during pyrolysis. Measurements were conducted at high temperatures (1260-1653 K) with high reactant compositions (favorable for $\mathrm{H}$-abstractions) under low pressure (Table 4). It was observed that the $\mathrm{CO}_{2}$ yields during pyrolysis were high and not strongly dependent on the aliphatic main chain length of the methyl ester. The incorporation of the theoretical work by Huynh and Violi [229] into the Fisher et al. model [208] allowed Farooq et al. [173] to account accurately for the $\mathrm{CO}_{2}$ yields experimentally observed, except at the highest temperatures. The theoretical work by Huynh and Violi [229], using ab initio techniques, resulted in the development of a new computed kinetic sub-model for MB thermal decomposition, including pyrolysis and oxidation processes. The rate constants for the unimolecular and bimolecular reactions in the temperature range of 300-2500 K were calculated using Rice-Ramsperger-Kassel-Marcus and transition-state theories, respectively. Because of the rather low barrier of the $\mathrm{H}$-abstraction reactions between $\mathrm{MB}$ and the flame radicals $\mathrm{H}, \mathrm{HO}$, and $\mathrm{CH}_{3}$, Huynh and Violi [229] focused their work on this class of reactions. Thirteen pathways (Fig. 8) were identified for $\mathrm{MB}$ thermal decomposition initiated by $\mathrm{H}$-abstraction reactions, which led to the formation of small species, such as $\mathrm{CH}_{3}, \mathrm{C}_{2} \mathrm{H}_{3}, \mathrm{CO}, \mathrm{CO}_{2}$, and formaldehyde $\left(\mathrm{H}_{2} \mathrm{CO}\right)$. Kinetic simulation results for high-temperature pyrolysis showed that the $\mathrm{H}+\mathrm{MB}$ reaction was the 
most important reaction in the initial stage of $\mathrm{MB}$ decomposition. In addition, the $\mathrm{C}(\mathrm{O}) \mathrm{OCH}_{3}=\mathrm{CO}+$ $\mathrm{CH}_{3} \mathrm{O}$ reaction was determined to be the main source of $\mathrm{CO}$ formation. Recently Farooq et al. [176] completed their study about the pyrolysis of $\mathrm{MB}$ by measuring concentration time-histories of $\mathrm{CO}$, $\mathrm{CO}_{2}, \mathrm{CH}_{3}$ and $\mathrm{C}_{2} \mathrm{H}_{4}$ using shock tube/laser absorption methods.

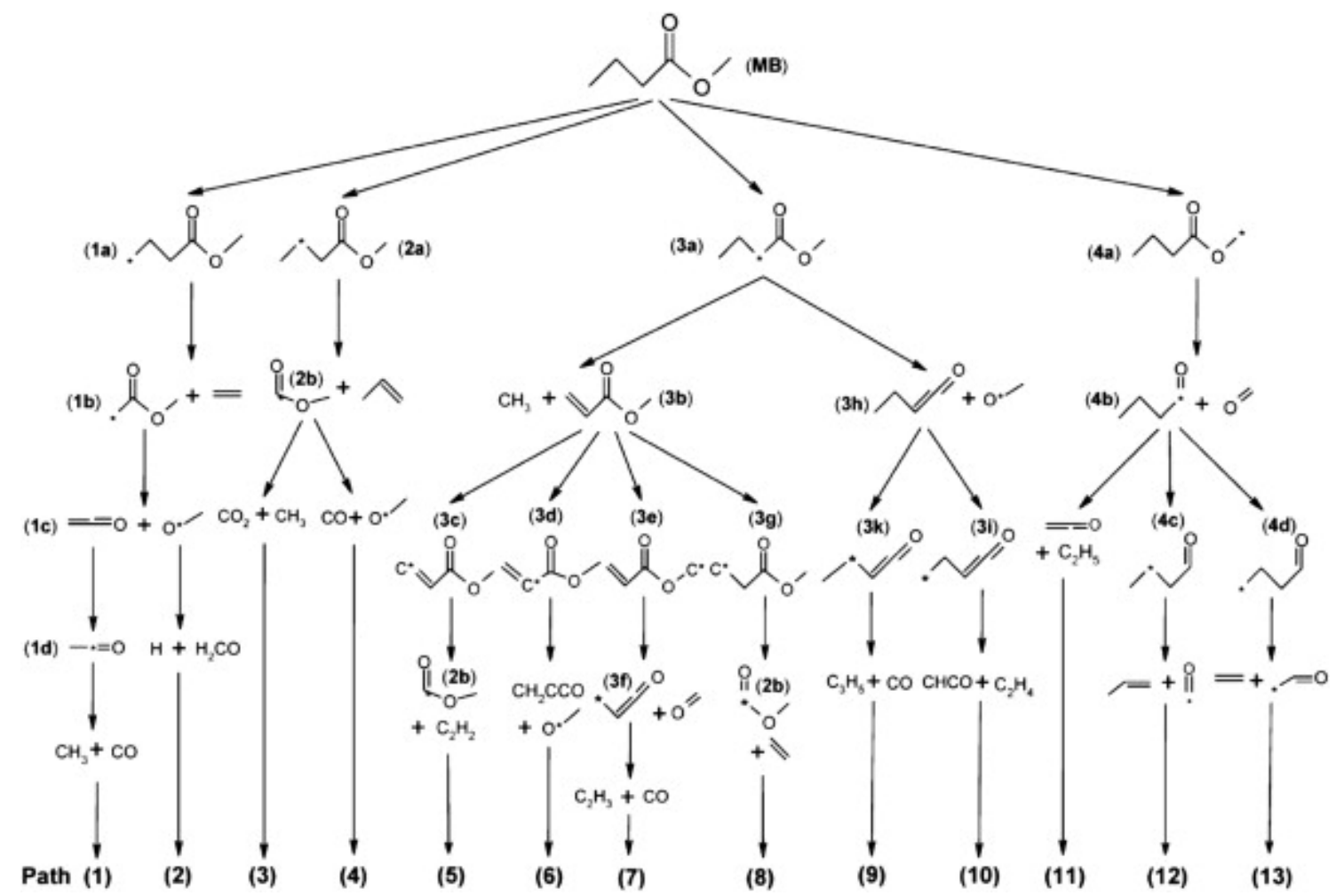

Figure 8 . The thirteen pathways for methyl butanoate thermal decomposition initiated by hydrogen abstraction reactions [229].

\subsubsection{Oxidation of methyl crotonate}

As biodiesel also contains fatty acid esters with one or two double bonds $(-\mathrm{CH}=\mathrm{CH}-)$, it is important to understand their impact on combustion chemistry. Therefore, various studies were conducted on methyl (E)-2-butenoate, common name methyl crotonate (MC), corresponding to one unsaturated $\mathrm{C}_{4}$-methyl ester $\left(\mathrm{CH}_{3} \mathrm{CH}=\mathrm{CH}(\mathrm{CO}) \mathrm{OCH}_{3}\right)$, for which oxidation behavior can be compared with the corresponding saturated C4-methyl ester $\mathrm{MB}\left(\mathrm{CH}_{3} \mathrm{CH}_{2} \mathrm{CH}_{2}(\mathrm{CO}) \mathrm{OCH}\right)$. In addition, these studies are helpful for increasing the amount of information related to $\mathrm{MB}$ oxidation because $\mathrm{MC}$ is an intermediate product formed during MB combustion $[166,174]$.

In their comparative study of $\mathrm{MC}$ and $\mathrm{MB}$ oxidation, Sarathy et al. [168] performed experiments in an OFDF and a JSR. Details related to the operating conditions are given in Table 4 . The mole fraction profiles of major intermediates, final products and reactants were measured. The authors observed that both fuel molecules had similar reactivity. Nevertheless, the experimental results showed that MC oxidation produced much higher levels of aldehydes (2-propenal and acetaldehyde: toxic products) and light unsaturated hydrocarbons (acetylene, propyne, 1-butene, and 1,3-butadiene: soot precursors) compared to MB. However, MB combustion had higher levels of ethylene $\left(\mathrm{C}_{2} \mathrm{H}_{4}\right)$. In addition, $\mathrm{MC}$ produced benzene, which was not detected for MB oxidation, in OFDF. These results led the authors to conclude that unsaturated esters would have a greater tendency to form soot than saturated esters. 
Later, Gaïl et al. [169] continued Sarathy et al.'s [168] work by extending the oxidation experiments made in a JSR for MC and MB (at atmospheric pressure over the temperature range of 850-1350 K and under stoichiometric conditions) to fuel-lean mixtures (Table 4). Furthermore, Gaill et al. [169] used these new experimental results in addition to those of Sarathy et al. [168] to extend the version of the detailed chemical kinetic model they previously proposed for MB oxidation [166] to MC by adding several specific reactions (Table 9). The rate constants of the new added reactions were derived using analogous reactions that occur in the established MB model. The thermochemical properties of the new species were calculated using THERGAS software [155], while the transport quantities were estimated from species of similar size and structure. The new revised mechanism consisted of 1516 reactions involving 301 species (Table 5). Overall, the new kinetic model reproduced the experimental data fairly well and confirmed the conclusions experimentally observed by Sarathy et al. [168].

Table 9. Reactions added to the Gaïl et al. model [166] for representing MC oxidation [169]. ${ }^{\text {a }}$

\begin{tabular}{|c|c|c|c|}
\hline Reaction & $\boldsymbol{A}$ & $n$ & $E_{a}$ \\
\hline $\mathrm{CH}_{3} \mathrm{CH}=\mathrm{CH}(\mathrm{C}=\mathrm{O}) \mathrm{O} .+. \mathrm{CH}_{3}=\mathrm{CH}_{3} \mathrm{CH}=\mathrm{CH}(\mathrm{C}=\mathrm{O}) \mathrm{OCH}_{3}$ & $8.00 \mathrm{E}+13$ & 0.00 & 0 \\
\hline $\mathrm{CH}_{3} \mathrm{CH}=\mathrm{CH} . \mathrm{C}=\mathrm{O}+\mathrm{CH}_{3} \mathrm{O} .=\mathrm{CH}_{3} \mathrm{CH}=\mathrm{CH}(\mathrm{C}=\mathrm{O}) \mathrm{OCH}_{3}$ & $6.00 E+13$ & 0.00 & 0 \\
\hline $\mathrm{CH}_{3} \mathrm{CH}=\mathrm{CH} .+\mathrm{CH}_{3} \mathrm{O} \cdot \mathrm{CO}=\mathrm{CH}_{3} \mathrm{CH}=\mathrm{CH}(\mathrm{C}=\mathrm{O}) \mathrm{OCH}_{3}$ & $8.00 E+13$ & 0.00 & 0 \\
\hline$. \mathrm{CH}_{2} \mathrm{CH}=\mathrm{CH}(\mathrm{C}=\mathrm{O}) \mathrm{OCH}_{3}=\mathrm{CH}_{2}=\mathrm{CH}-\mathrm{CH}(\mathrm{C}=\mathrm{O}) \mathrm{OCH}_{3}$ & $4.00 \mathrm{E}+12$ & 0.00 & 60,000 \\
\hline $\mathrm{CH}_{3} \mathrm{CH}=\mathrm{CH}(\mathrm{C}=\mathrm{O}) \mathrm{OCH}_{3}+. \mathrm{CH}_{3}=\mathrm{CH}_{3} \mathrm{CH}=\mathrm{CH}(\mathrm{C}=\mathrm{O}) \mathrm{OCH}_{2} .+\mathrm{CH}_{4}$ & $1.00 \mathrm{E}+12$ & 0.00 & 7300 \\
\hline $\mathrm{CH}_{3} \mathrm{CH}=\mathrm{CH}(\mathrm{C}=\mathrm{O}) \mathrm{OCH}_{2}+\mathrm{H} .=\mathrm{CH}_{3} \mathrm{CH}=\mathrm{CH}(\mathrm{C}=\mathrm{O}) \mathrm{OCH}_{3}$ & $1.00 \mathrm{E}+14$ & 0.00 & 0 \\
\hline $\mathrm{CH}_{3} \mathrm{CH}=\mathrm{CH}(\mathrm{C}=\mathrm{O}) \mathrm{OCH}_{2} .=\mathrm{CH}_{3} \mathrm{CH}=\mathrm{CH} . \mathrm{C}=\mathrm{O}+\mathrm{CH}_{2} \mathrm{O}$ & $5.01 \mathrm{E}+12$ & 0.00 & 19,000 \\
\hline $\mathrm{CH}_{3} \mathrm{CH}=\mathrm{CH}(\mathrm{C}=\mathrm{O}) \mathrm{OCH}_{2} .+\mathrm{OH}=\mathrm{CH}_{3} \mathrm{CH}=\mathrm{CH}(\mathrm{C}=\mathrm{O}) \mathrm{OCH}_{3}+. \mathrm{O}$. & $3.50 \mathrm{E}+11$ & 0.00 & 29,900 \\
\hline $\mathrm{CH}_{3} \mathrm{CH}=\mathrm{CH}(\mathrm{C}=\mathrm{O}) \mathrm{OCH}_{3}+. \mathrm{CH}_{3}=. \mathrm{CH}_{2} \mathrm{CH}=\mathrm{CH}(\mathrm{C}=\mathrm{O}) \mathrm{OCH}_{3}+\mathrm{CH}_{4}$ & $1.00 \mathrm{E}+12$ & 0.00 & 7300 \\
\hline $\mathrm{CH}_{3} \mathrm{CH}=\mathrm{CH}(\mathrm{C}=\mathrm{O}) \mathrm{OCH}_{3}+\mathrm{H} .=. \mathrm{CH}_{2} \mathrm{CH}=\mathrm{CH}(\mathrm{C}=\mathrm{O}) \mathrm{OCH}_{3}+\mathrm{H}_{2}$ & $3.70 E+13$ & 0.00 & 3900 \\
\hline $\mathrm{CH}_{3} \mathrm{CH}=\mathrm{CH}(\mathrm{C}=\mathrm{O}) \mathrm{OCH}_{3}+\mathrm{O}_{2}=. \mathrm{CH}_{2} \mathrm{CH}=\mathrm{CH}(\mathrm{C}=\mathrm{O}) \mathrm{OCH}_{3}+\mathrm{HO}_{2}$. & $3.00 \mathrm{E}+13$ & 0.00 & 52,800 \\
\hline$. \mathrm{CH}_{2} \mathrm{CH}=\mathrm{CH}(\mathrm{C}=\mathrm{O}) \mathrm{OCH}_{3}+. \mathrm{OH}=\mathrm{CH}_{3} \mathrm{CH}=\mathrm{CH}(\mathrm{C}=\mathrm{O}) \mathrm{OCH}_{3}+. \mathrm{O}$. & $7.00 \mathrm{E}+11$ & 0.00 & 29,900 \\
\hline $\mathrm{CH}_{3} \mathrm{CH}=\mathrm{CH}(\mathrm{C}=\mathrm{O}) \mathrm{OCH}_{3}+. \mathrm{OH}=. \mathrm{CH}_{2} \mathrm{CH}=\mathrm{CH}(\mathrm{C}=\mathrm{O}) \mathrm{OCH}_{3}+\mathrm{H}_{2} \mathrm{O}$ & $3.00 \mathrm{E}+13$ & 0.00 & 1230 \\
\hline $\mathrm{CH}_{3} \mathrm{CH}=\mathrm{CH}(\mathrm{C}=\mathrm{O}) \mathrm{OCH}_{3}+\mathrm{HO}_{2} .=. \mathrm{CH}_{2} \mathrm{CH}=\mathrm{CH}(\mathrm{C}=\mathrm{O}) \mathrm{OCH}_{3}+\mathrm{H}_{2} \mathrm{O}_{2}$ & $1.50 \mathrm{E}+11$ & 0.00 & 14,190 \\
\hline$. \mathrm{CH}_{2} \mathrm{CH}=\mathrm{CH}(\mathrm{C}=\mathrm{O}) \mathrm{OCH}_{3}+\mathrm{H} .=\mathrm{CH}_{3} \mathrm{CH}=\mathrm{CH}(\mathrm{C}=\mathrm{O}) \mathrm{OCH}_{3}$ & $5.00 \mathrm{E}+12$ & 0.00 & 0 \\
\hline $\mathrm{CH}_{2} \mathrm{CH}=\mathrm{CH}(\mathrm{C}=\mathrm{O}) \mathrm{OCH}_{3}+. \mathrm{O} .=. \mathrm{CH}=\mathrm{CH}(\mathrm{C}=\mathrm{O}) \mathrm{OCH}_{3}+\mathrm{CH}_{2} \mathrm{O}$ & $1.58 \mathrm{E}+07$ & 1.80 & -1216 \\
\hline $\mathrm{CH}_{3} \mathrm{CH}=\mathrm{CH}(\mathrm{C}=\mathrm{O}) \mathrm{OCH}_{3}+. \mathrm{OH}=\mathrm{CH}_{3} \mathrm{CH}=\mathrm{CH}(\mathrm{C}=\mathrm{O}) \mathrm{OCH}_{2} .+\mathrm{H}_{2} \mathrm{O}$ & $3.00 \mathrm{E}+13$ & 0.00 & 1230 \\
\hline $\mathrm{CH}_{3} \mathrm{CH}=\mathrm{CH}(\mathrm{C}=\mathrm{O}) \mathrm{OCH}_{3}+\mathrm{H} .=\mathrm{CH}_{3} \mathrm{CH}=\mathrm{CH}(\mathrm{C}=\mathrm{O}) \mathrm{OCH}_{2} .+\mathrm{H}_{2}$ & $3.70 E+13$ & 0.00 & 3900 \\
\hline
\end{tabular}

${ }^{\mathrm{a}}$ The rate coefficients are listed in the generalized Arrhenius form ( $\left.\mathrm{K}=\mathrm{ATn} \cdot \exp (\mathrm{Ea} / \mathrm{RT})\right)$ where units are mol, $\mathrm{cm}, \mathrm{s}, \mathrm{K}$, and cal.

As for $\mathrm{C}_{5} \mathrm{H}_{10} \mathrm{O}_{2}$ isomers (MB, methyl iso-butanoate and EP, Section 3.4.1) [180], Yang et al. [181] performed the experimental and modeling study of the oxidation of three unsaturated $\mathrm{C}_{5} \mathrm{H}_{8} \mathrm{O}_{2}$ isomers: $\mathrm{MC}$, methyl methacrylate and ethyl propenoate in a premixed flame (using tunable synchrotron vacuum ultraviolet photo-ionization mass spectrometry). The authors observed that the presence of the double bond in esters enhances the formation of oxygenated intermediates in flames.

\subsubsection{Oxidation of model molecules suitable as biodiesel surrogates}

Although investigations related to $\mathrm{C}_{4}$-methyl esters helped to add the knowledge regarding the impact of the methyl ester group on combustion chemistry, the molecules that were first selected as biodiesel surrogates were determined to be unsuitable. In particular, no evidence of cool flame or NTC region (except for a few sources claiming weak NTC behavior $[168,208]$ ) was experimentally observed or predicted by modeling. Nevertheless, actual biodiesel components, as with long-chain alkanes, should show these two types of phenomena (indicating competition between temperaturedependent oxidation channels, Fig. 4). It is now admitted that this deficiency is encountered with $\mathrm{C}_{4}$-methyl esters because their aliphatic main chains are too short. In such circumstances, peroxy radicals $\mathrm{RO}_{2}$ (reaction 3, Fig. 4) react to yield hydroperoxy radicals $\mathrm{QOOH}$ (reaction 4, Fig. 4) via internal $\mathrm{H}$-atom transfer through a cyclic transition state with high ring strain energy. Methyl 
hexanoate or methyl octanoate, and a fortiori methyl decanoate, methyl-5- and methyl-9-decenoate, were determined to be more suitable biodiesel surrogates (although their molecular structure prevents them from performing as well as actual biodiesel components [230]).

\subsubsection{Oxidation of methyl esters from methyl hexanoate to methyl octanoate}

Dayma et al. [182] performed the study of the oxidation of methyl hexanoate (MHX) in a JSR at high pressure (10 atm) and over a range of low to high temperatures $(500-1000 \mathrm{~K})$ at various equivalence ratios and a constant residence time (Table 4). Concentration (mole fraction) profiles of 23 species were measured $\left(\mathrm{O}_{2}, \mathrm{H}_{2}, \mathrm{CO}, \mathrm{CO}_{2}\right.$, formaldehyde, methane, acetaldehyde, ethane, ethene, acetylene, propene, propanal, propenal, 1-butene, 1-pentene, and unsaturated methyl esters from $C_{4}$ to $C_{7}$ ). Under the investigated operating conditions, the authors reported that MHX showed three main reaction regimes similar to large hydrocarbons:

- A cool flame zone (560-660 K) characterized by fuel consumption at low temperatures and the production of small aldehydes and methyl hexenoates.

- A NTC zone (660-760 K) where the total reactivity decreased with increasing temperature.

- A high-temperature zone ( $>760 \mathrm{~K})$ with the total consumption of the fuel.

This new set of experimental data was used by the authors to develop and validate a detailed chemical kinetic model for MHX oxidation, which consisted of 435 species and 1875 reactions (Table 5). The mechanism was generated using a hierarchical and systematic method. The first edifice was based on the comprehensive MB oxidation mechanism developed by Fisher et al. [208], which was added to a 1-butene submechanism validated under various experimental conditions [223]. Nevertheless, four reactions from the Fisher et al. model [208] were modified to obtain better predictions, particularly for ethylene $\left(\mathrm{C}_{2} \mathrm{H}_{4}\right)$ and methyl-2-propenoate $\left(\mathrm{CH}_{2}=\mathrm{CHC}(=\mathrm{O}) \mathrm{OCH}_{3}\right)$ profiles (Table 10). In addition, a submechanism of 551 reactions was implemented to model the oxidation of $\mathrm{MHX}$ and related compounds from low to high temperatures. For similar reactions, the rate constants were those proposed by Fisher et al. [208] for MB. Nevertheless, because of the larger length of the aliphatic main chain for $\mathrm{MHX}$, some rate constants had to be taken from the literature using structure-reactivity relationships (with computation of the reverse rate constants from the corresponding forward rate constants and appropriate equilibrium constants $K_{c}=k_{\text {forward }} / k_{\text {reverse }}$ calculated from thermo-chemistry). Thermochemical data were taken from the Fisher et al. model [208] and estimated using THERGAS software [155] for species belonging to MHX submechanism. The kinetic modeling was performed using the PSR code of CHEMKIN [158]. The proposed kinetic model yielded good overall agreement with the experimental results. Using reaction path analysis (Fig. 9), the authors highlighted that the oxidation behavior of MHX was mainly controlled, over the investigated temperature and pressure ranges, by the weakness of the $\mathrm{C}-\mathrm{H}$ bond on the carbon adjacent to the methyl ester group. Dayma et al. [183] also performed the experimental and modeling study of the oxidation of MH in a JSR following the same strategy as the one used for MHX [182]. This study confirmed the conclusions that were drawn for MHX.

HadjAli et al. [172] examined the autoignition of five linear methyl esters from butanoic acid to octanoic acid in a rapid compression machine (RCM) at operating conditions useful for compressionignition engine model validation (for low and intermediate temperatures (650-850 K) and medium to high pressures (4-20 bar), and for stoichiometric mixtures and the same dilution of oxygen as in air; Table 4). Nevertheless, under the conditions investigated, the vapor pressures of methyl heptanoate and octanoate were too low to permit valid autoignition experiments. Therefore, the authors selected MHX for a full investigation of its autoignition properties, including the identification and quantification of the intermediate products at low-temperature oxidation. The oxidation scheme and overall reactivity of $\mathrm{MHX}$ were examined and compared to the reactivity of $\mathrm{C}_{4}-\mathrm{C}_{7} \mathrm{n}$-alkanes under the same experimental conditions to evaluate the impact of the methyl ester function on the reactivity of the $n$-alkyl chain. The low-temperature reactivity of MHX that leads to the first stage of autoignition 
(cool flame event) was similar to n-heptane. However, the NTC region was located at a lower temperature than in $\mathrm{n}$-pentane and $\mathrm{n}$-butane. The authors also presented the main reaction pathways that led to the detected products (for the lighter species: acetic acid, propenal, 1-pentene, propanal, methyl acetate, butanone, and butanal; for the $\mathrm{C}_{4}-\mathrm{C}_{7}$ species: unsaturated methyl esters, methyl epoxy esters with 3-5 atom rings, and 5-butyl-1,3-dioxolane-4-one).

Table 10. Reactions and rate constants modified by Dayma et al. [182] (bold) in MB Fisher et al. model [208]. ${ }^{\text {a }}$

\begin{tabular}{|c|c|c|c|}
\hline Reaction & $A$ & $n$ & $E_{a}$ \\
\hline $\mathrm{C}_{2} \mathrm{H}_{4}+. \mathrm{C}(=\mathrm{O}) \mathrm{OCH}_{3}=. \mathrm{CH}_{2} \mathrm{CH}_{2}(\mathrm{C}=\mathrm{O}) \mathrm{OCH}_{3}$ & $2.11 \mathrm{E}+11$ & 0.00 & 3350 \\
\hline$. \mathrm{CH}_{2} \mathrm{CH}_{2} \mathrm{C}(=\mathrm{O}) \mathrm{OCH}_{3}=\mathrm{C}_{2} \mathrm{H}_{4}+. \mathrm{C}(=0) \mathrm{OCH}_{3}$ & $2.00 E+13$ & 0.00 & 30,500 \\
\hline $\mathrm{CH}_{2}=\mathrm{CHC}(=\mathrm{O}) \mathrm{OCH}_{3}+\mathrm{H} .=. \mathrm{CH}_{2} \mathrm{CH}_{2} \mathrm{C}(=\mathrm{O}) \mathrm{OCH}_{3}$ & $1.00 \mathrm{E}+13$ & 0.00 & 2900 \\
\hline$. \mathrm{CH}_{2} \mathrm{CH}_{2} \mathrm{C}(=\mathrm{O}) \mathrm{OCH}_{3}=\mathrm{H} .+\mathrm{CH}_{2}=\mathrm{CHC}(=0) \mathrm{OCH}_{3}$ & $3.00 \mathrm{E}+13$ & 0.00 & 37,500 \\
\hline $\mathrm{CH}_{2}=\mathrm{CHC}(=\mathrm{O}) \mathrm{OCH}_{3}+. \mathrm{OH} \rightarrow \mathrm{CH}_{2} \mathrm{O}+\mathrm{C}_{2} \mathrm{H}_{3} \mathrm{CO}+\mathrm{H}_{2} \mathrm{O}$ & $5.25 \mathrm{E}+09$ & 0.97 & 1590 \\
\hline $\mathrm{CH}_{2}=\mathrm{CHC}(=0) \mathrm{OCH}_{3}+. \mathrm{OH} \rightarrow . \mathrm{CH}_{2} \mathrm{C}(=\mathrm{O}) \mathrm{OCH}_{3}+\mathrm{CH}_{2} \mathrm{O}$ & $4.00 \mathrm{E}+12$ & 0.00 & -928 \\
\hline $\mathrm{CH}_{2}=\mathrm{CHC}(=0) \mathrm{OCH}_{3}+. \mathrm{OH} \rightarrow .0 . \mathrm{CHC}(=0) \mathrm{OCH}_{3}+. \mathrm{CH}_{3}$ & $6.90 E+11$ & 0.00 & -928 \\
\hline $\mathrm{C}_{2} \mathrm{H}_{4} \mathrm{OH}=\mathrm{C}_{2} \mathrm{H}_{4}+. \mathrm{OH}$ & $1.293 E+12$ & -0.37 & 26,850 \\
\hline $\mathrm{C}_{2} \mathrm{H}_{4}+. \mathrm{OH}=\mathrm{CH}_{2} \mathrm{O}+. \mathrm{CH}_{3}$ & $1.40 \mathrm{E}+12$ & 0.00 & 3250 \\
\hline
\end{tabular}

${ }^{\mathrm{a}}$ The rate constants were expressed using the modified Arrhenius equation $\mathrm{k}=\mathrm{ATn} \cdot \exp (\mathrm{Ea} / \mathrm{RT})$ with units $\mathrm{cm}, \mathrm{mol}, \mathrm{s}, \mathrm{K}$, and cal.

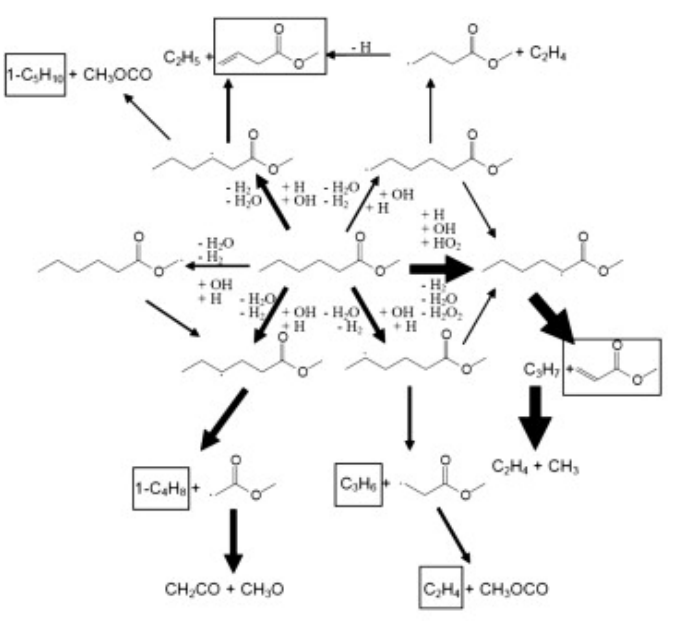

(a)

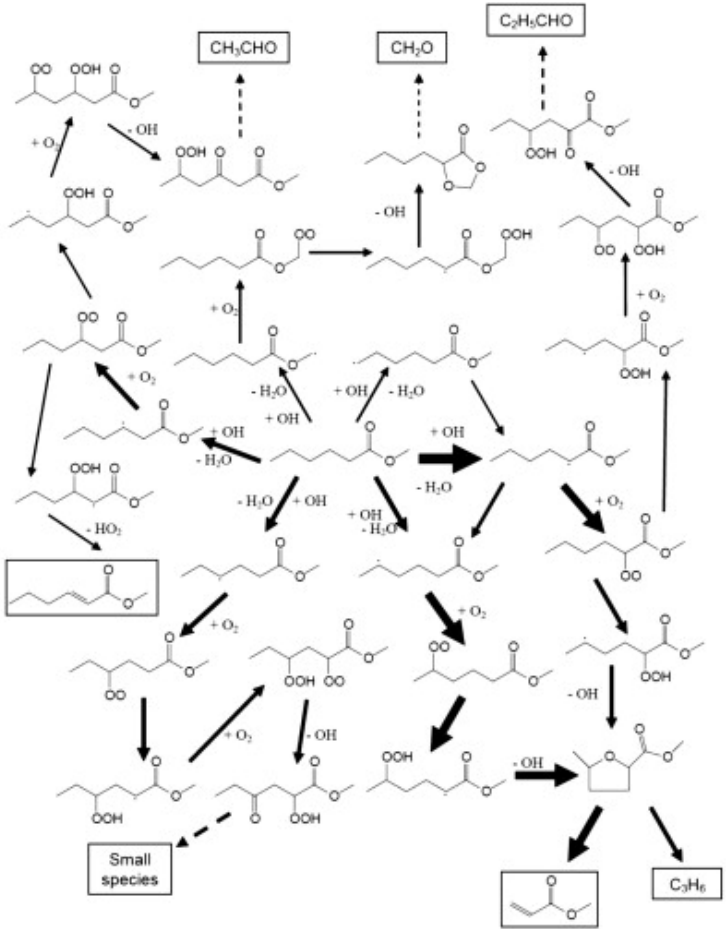

(b)

Figure 9. Reaction path analysis for the oxidation of methyl hexanoate in JSR for two operating conditions (a) $T=950 \mathrm{~K}, \phi=1, \tau=1 \mathrm{~s}$, and $\mathrm{P}=10 \mathrm{~atm}$; (b) $\mathrm{T}=650 \mathrm{~K}, \phi=1, \tau=1 \mathrm{~s}$, and $\mathrm{P}=10 \mathrm{~atm}$ [182]. The thickness of the arrows is proportional to the importance of the reaction path. The framed products were quantified (dashed arrows mean a several-step production).

Concerning oxidation of methyl octanoate (MOC) under atmospheric pressure, new experimental results related to concentration profiles of stable species (reactants, intermediates, and final products) were obtained by Dayma et al. [184] using two different types of equipment: in a JSR as a function of temperature (from 800 to $1350 \mathrm{~K}$ ) and in an OFDF as a function of distance from the fuel port (Table 4). Experimentally, atmospheric MOC oxidation in the JSR does not show any cool flame 
or NTC behavior, whereas hot ignition occurs at approximately $800 \mathrm{~K}$. A detailed kinetic model for MOC oxidation was developed in the study, including 383 species and 2781 reactions (Table 5). Based on a strong hierarchical structure, the kinetic model for MOC oxidation was derived from the model previously proposed by the same authors for MHX oxidation with some changes: (i) only the hightemperature chemistry was included because no cool flame was detected in the MOC JSR experiments and (ii) some rate constants were reevaluated and updated according to the most recent literature. These updates particularly concern $\mathrm{H}$-abstractions with peroxy radicals, $\beta$-scission by $\mathrm{Csp}^{3}-\mathrm{Csp}^{3}$ bond breaking, $\mathrm{C}-\mathrm{O}$ bond rupture yielding methoxy, $\mathrm{H}$-transfer through $5_{-}, \mathrm{C}_{-}$, and $7-$ membered ring transition states, the $\mathrm{C}_{0}-\mathrm{C}_{3}$ submechanism as a whole, and unimolecular ignitions by $\mathrm{C}-\mathrm{C}$ bond rupture, the sensitivity of which increased with diminishing pressure. Indeed, $\mathrm{MHX}$ oxidation measurements and modeling were performed by Dayma et al. [182] under higher pressure conditions (10 atm) for which unimolecular initiations by $C-C$ bond ruptures were not sensitive. In addition to the transport property database used by Seshadri et al. [188], new values were estimated for stable $\mathrm{C}_{2}-\mathrm{C}_{10}$ saturated and unsaturated methyl esters and their corresponding radicals by assuming that the transport properties were similar for saturated and unsaturated esters of the same chain length. Model validation for MOC oxidation was performed by the authors using the new measurements, which showed reasonable agreement between the simulation results and hightemperature experimental data. In addition, the reaction pathway proposed by Dayma et al. [184] for MOC oxidation was fairly similar to the pathway proposed by the same authors for MHX at $950 \mathrm{~K}$ and $10 \mathrm{~atm}$ (Fig. 9), with the same predominant routes. The model developed by Dayma et al. [184] was used by Rotavera and Petersen [185] to perform comparisons with ignition delay times obtained using a ST. A good agreement was obtained between the two sets of data at 1.5 bar for lean mixtures whereas the model was not able to reproduce the experimental ignition delay time for stoichiometric and rich mixtures at 1.5 and 10 bar.

\subsubsection{Oxidation of methyl decanoate}

Szybist et al. $[186,187]$ reported the autoignition behavior of methyl decanoate (MD) in a variable compression ratio $(\mathrm{CR})$ octane rating engine charged with premixed MD and air. The spark plug was disabled for this study so that combustion could be initiated kinetically, and ignition occurred simultaneously at multiple points throughout the cylinder, as in $\mathrm{HCCl}$ combustion. The CR of the engine was adjusted over a range of 4.0-13.75 at multiple equivalence ratios (Table 4). During each $\mathrm{CR}$ sweep, the exhaust composition of $\mathrm{CO}, \mathrm{CO}_{2}$, formaldehyde, and acetaldehyde was monitored using Fourier transformed-infrared (FTIR) spectrometry and condensable exhaust gas was collected for subsequent gas chromatography/mass spectrometry (GC-MS) analysis. The authors observed that MD exhibited two-stage ignition with LTHR followed by the main combustion event or HTHR for sufficiently high compression ratios ( $C R \approx 9.1$ ). For lower compression ratios ( $C R \approx 5.6$ ), MD only underwent LTHR. GC-MS speciation of the LTHR exhaust condensate revealed the formation of various classes of species, a selection of which are listed in Table 11. In addition to a number of saturated and unsaturated methyl esters with shorter aliphatic main chains than MD, a number of saturated oxo-methyl esters with an additional carbonyl group on the aliphatic main chain was also identified. This result revealed that the long-aliphatic main chain acts similarly to $n$-paraffins during LTHR, while the ester group remains intact. However, FTIR analysis revealed significant amounts of $\mathrm{CO}_{2}$ produced during LTHR. Based on the commonly established results, according to which oxidation of $\mathrm{CO}$ to $\mathrm{CO}_{2}$ does not occur under low-temperature conditions when hydrocarbon chains are present in the medium [231], the authors concluded that the $\mathrm{CO}_{2}$ produced by MD during LTHR was because of decarboxylation of the ester group. Thus, from FTIR and GC-MS information, the decarboxylation of MD should not occur until the aliphatic main chain has been largely consumed by LTHR reactions, which incorporate the contribution of oxygen in the molecule. The authors highlight that the oxygen present in the fuel is used less effectively to remove carbon from the pool of soot precursors, which reveals that esters should reduce particulate matter emissions less efficiently than 
ethers because of the loss of $\mathrm{CO}_{2}$ directly from the decomposition of the parent molecule (and not by oxidation). This feature will be further discussed in Section 4.1.

Table 11. Selected identified species from the low-temperature heat release exhaust condensate of methyl decanoate oxidation [186] and [187]. ${ }^{\mathrm{a}}$

\begin{tabular}{ll}
\hline \multicolumn{1}{c}{ Methyl esters } & \multicolumn{1}{c}{ Oxo-methyl esters } \\
\hline 2-methyl butanoic acid methyl ester & 4-oxopentanoic acid methyl ester \\
Methyl hexanoate & 5-oxopentanoic acid methyl ester \\
Methyl heptenoate & 5-oxohexanoic acid methyl ester \\
Methyl octenoate & 6-oxoheptanoic acid methyl ester \\
Methyl octanoate & 4-oxooctanoic acid methyl ester \\
Methyl nonenoate (isomers) & 2-oxodecanoic acid methyl ester \\
Methyl nonanoate & 9-oxodecanoic acid methyl ester \\
Methyl decenoate (isomers) & \\
Methyl decanoate &
\end{tabular}

In a complementary approach to the experimental work by Szybist et al. $[186,187]$, Herbinet et al. [142] developed a detailed kinetic model for MD oxidation by combining the mechanisms previously proposed for $n$-heptane, iso-octane $[217,207]$ and MB [208] with the low- and high-temperature chemistry specific to MD. Kinetic parameters and thermochemical properties were updated according to the recent literature, including quantum theory-based estimations. In particular, the C$\mathrm{H}$ bond dissociation energy (bond enthalpy) of the carbon atom adjacent to the carbonyl group has been updated according to El-Nahas et al. [225], which was previously performed by Metcalfe et al. [167] and Dooley et al. [170] (value adopted by Herbinet et al. [142] and obtained by El-Nahas et al. [225] from ab initio estimations: $\left.94.1 \mathrm{kcal} \mathrm{mol}^{-1}\right)$. Based on the overall primary oxidation reaction pathways and reaction classes derived from the work by Curran et al. $[207,217]$ and extended to methyl and ethyl esters (Fig. 4 and Table 6, respectively), the resulting mechanism proposed by Herbinet et al. [142] included 8820 reactions and 3012 species (Table 5). The large numbers of reactions and species result mainly from the fact that $M D$ is not a symmetric molecule (unlike an $n$ alkane). Also, numerous types of reactions were accounted for. In particular, the isomerization of $\mathrm{RO}_{2}$ to $\mathrm{QOOH}$ radicals in the low-temperature regime (reaction 4 in Fig. 4) was considered as proceeding through 5-, 6-, 7-, and 8-membered cyclic transition states yielding numerous permitted $\mathrm{H}$-shifts. Model validation was conducted by comparing the computed results with various classes of experimental information ranging from low- to high-temperature regions: the only available experimental data for MD obtained in a motored engine $[186,187]$ as well as with rapeseed methyl ester oxidation results in a JSR [197] and n-decane ignition in shock tubes [232]. The first two classes of experimental information yielding species profiles helped to highlight the model's ability to reproduce the chemistry of most products formed, particularly the early production of $\mathrm{CO}_{2}$ that is specific to biodiesel. This important feature of the mechanism was obtained because of the insertion of low-temperature reactions that lead to the formation of $\mathrm{CO}_{2}$. The majority of these reactions is listed in Table 12. Reactions 1 and 4 (Table 12) are uniquely derived from the methyl ester group in $M D$ and would not occur in hydrocarbon oxidation. In addition, the authors noted that the kinetic parameters were updated according to the work by Glaude et al. [233] relative to dimethyl carbonate in reaction 4 of Table 12 . Successive reactions from an alkyl-ester radical to the formation of $\mathrm{CO}_{2}$ through the $\mathrm{OCHO}$ radical were also suggested by the authors; they are reported in Fig. 10. As shown, one oxygen atom in $\mathrm{CO}_{2}$ is derived from the non-carbonyl part of the ester group, and the other oxygen atom is derived from the oxygen molecule involved in the addition reaction. Nevertheless, Hayes and Burgess [234] highlighted using quantum-chemistry theory (ab initio and density functional theory) that this proposed $\mathrm{CO}_{2}$ production pathway is but one hypothesis. Other peroxy radical reactions may contribute to or even dominate in $\mathrm{MB}$ oxidation. Moreover, $\mathrm{CO}_{2}$ production in large methyl esters may vary further from the pathway proposed for MB; the ring size 
of the transition state (resulting from the aliphatic main chain length) and the type of the $\mathrm{H}$-atom abstracted both play a role in this process.

Table 12. Main reactions leading to the formation of $\mathrm{CO}_{2}$ at low temperature (from MD oxidation model by Herbinet et al. [142]).
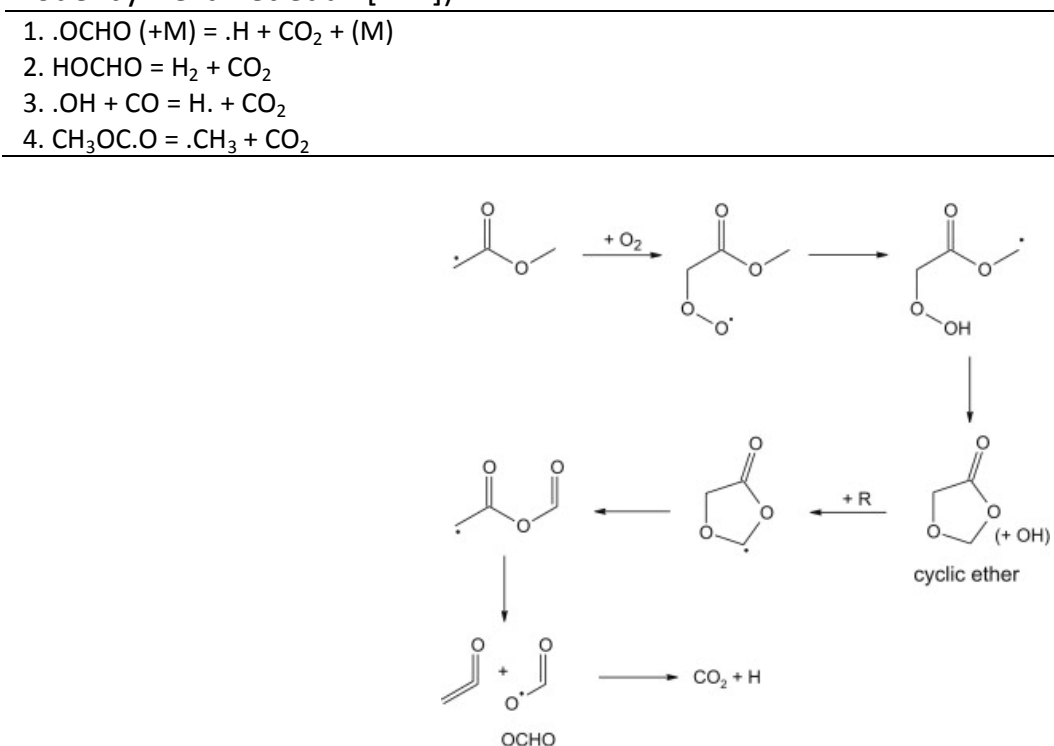

Figure 10. Successive reactions from an alkyl-ester radical to the formation of $\mathrm{CO}_{2}$ via the radical $\mathrm{OCHO}$ (from MD oxidation model by Herbinet et al. [142]).

In addition, Herbinet et al. [142] obtained results that were in good agreement with $\mathrm{n}$-decane experiments in shock tubes (ignition delay times and OH profiles) with an NTC region occurring in the range $780-920 \mathrm{~K}$ at $12 \mathrm{~atm}$. This result shows that the reactivity of large methyl esters is similar to that of $\mathrm{n}$-alkanes of similar size. Nevertheless, fine kinetic details, such as early $\mathrm{CO}_{2}$ production occurring at low temperatures during biodiesel combustion, can only be predicted by considering actual methyl ester fuels.

Following this work, an experimental and kinetic modeling study of the extinction and ignition of MD in non-premixed, aerodynamically strained flows was investigated by Seshadri et al. [188]. These characteristics were investigated under such conditions because flame extinction in highly strained flows is an important problem in gas turbine engines and because fuel/air mixtures must be ignited in flows in internal combustion engines. Critical conditions of counter flow extinction and ignition were measured with a fuel stream comprised of vaporized MD in nitrogen and an oxidizer stream of air (Table 4). The detailed chemical kinetic model for MD by Herbinet et al. [142] unfortunately includes too large a number of reactions and species for direct use in current flame codes. Therefore, a skeletal mechanism was deduced from the detailed mechanism using the directed-relation graph method. The derived skeletal mechanism only included 713 elementary reactions and 125 species. Predictions of extinction and ignition of MD in non-premixed counter flows agreed with the experimental data. In addition, the authors noted that the derived skeletal mechanism showed the minor importance of the low-temperature chemistry under the considered counter flow conditions. The reaction chemistry of most importance was determined to be the high-temperature reactions of fuel decomposition, radical abstraction, isomerization, and radical decomposition. The formation and consumption of olefin intermediates with ester moieties were also found to be significant.

The same year, Hoffman and Abraham [212] investigated ignition delay and NO formation rates for $\mathrm{MD}$ and $\mathrm{n}$-heptane (mineral diesel surrogate) under conditions of varying $\mathrm{O}_{2}$ concentration by employing the CHEMKIN homogeneous constant-pressure reactor model and the homogeneous- 
charge compression-ignition engine model [235]. Ignition delay time was defined in this study as the time for the gas mixture to reach $1500 \mathrm{~K}$ in the homogeneous reactor. Calculations were performed in a wide temperature range $(700-1350 \mathrm{~K}$ ) at two pressures (12.5 and $40 \mathrm{~atm}$ ) using stoichiometric mixtures of fuel and air $\left(21 \%\right.$ in $\left.\mathrm{O}_{2}\right)$. Reduced $\mathrm{O}_{2}$ molar concentrations $(18 \%, 15 \%$, and $12 \%)$ were obtained by replacing a portion of the initial $\mathrm{O}_{2}$ oxidizer with N2. This procedure was adopted to simulate the dilution effect of exhaust-gas recirculation. The MD detailed chemical kinetic model proposed by Herbinet et al. [142] was used without any modification. Nevertheless, the GRI-Mech 3.0 mechanism [211], involving 105 reactions and 20 species, was added to predict NO formation (Table 5). Computed results led the authors to observe that reducing $\mathrm{O}_{2}$ concentrations increased ignition delay for all fuels. As previously observed by Herbinet et al. [142] for MD and n-decane, MD and $n$-heptane showed similar autoignition characteristics, but with the NTC region occurring in different temperature ranges (in the range $750-900 \mathrm{~K}$ at $12.5 \mathrm{~atm}$ and $820-950 \mathrm{~K}$ at $40 \mathrm{~atm}$ for MD and at $50 \mathrm{~K}$ higher for $\mathrm{n}$-heptane). Ignition delays were roughly the same for $\mathrm{n}$-heptane and MD in the NTC region; however, outside the NTC region, the delay for MD was 30-60\% shorter. The authors also highlighted the effect of pressure on the NTC region. They observed that at higher pressures, the NTC region shifted toward higher temperatures and became less pronounced (because of $\mathrm{H}_{2} \mathrm{O}_{2}$ dissociation in $2 \mathrm{HO}$ at lower temperatures when pressure was increased). Furthermore, the addition of NO to simulate exhaust-gas recirculation reduced the ignition delay time. However, in practical applications, the increase in ignition delay because of lower $\mathrm{O}_{2}$ concentrations dominated any decrease because of NO addition.

More recently, Sarathy et al. [189] performed the first combustion data for MD in an OFDF (Table 4). Among the experimentally determined species profiles, the production of $C_{5}-C_{8}$ 1-alkenes, which were formed after $\beta$-scission of fuel radicals, was of particular interest. The production of lowmolecular-weight oxygenated compounds, such as formaldehyde, ketene, and isomers of $\mathrm{C}_{2} \mathrm{H}_{4} \mathrm{O}$ (acetaldehyde and ethenol) resulting from the decomposition of the ester moiety in $\mathrm{MD}$, was also observed. Furthermore, the experimental data were used to develop a novel skeletal mechanism based on the approach of Seshadri et al. [188] with minor modifications of the detailed model proposed by Herbinet et al. [142]. Similar to Seshadri et al. [188], Sarathy et al. [189] observed that the consumption of fuel was dominated by high-temperature chemical reactions. The resulting skeletal mechanism (648 species and 2998 reactions) was first validated by the authors to reproduce the behavior of the improved version of Herbinet et al. model [142] when applied to a PSR operating at low temperatures (500-950 K), elevated pressure (100 atm), and stoichiometric conditions and was successfully used to predict the OFDF measurements of MD (Table 5). This result highlights the effectiveness of the directed-relation graph method in producing a mechanism that is computationally practical for one-dimensional flame simulations yet also retains a level of chemical fidelity. To complete the work by Sarathy et al. [189], Glaude et al. [153] performed oxidation of MD in JSR at temperatures from 500 to $1100 \mathrm{~K}$, including the NTC region, under stoichiometric conditions and atmospheric pressure (Table 4). Over 30 reaction products, including olefins, unsaturated esters, and some cyclic ethers, were quantified and successfully simulated through a new detailed chemical kinetic mechanism (Ref. [213] in Table 5) and automatically generated using a new version of EXGAS software extended to large methyl esters [152,153] (further details of this version are presented in Section 3.4.6). In addition, the proposed mechanism was revealed to be of the same accuracy level as the model by Herbinet et al. [142], which is based on the same general rules (Section 3.2) but used a different generation approach (Section 3.1.1).

Furthermore, Ahmed [236] simulated the effect of $\mathrm{MD}$ addition to petrodiesel surrogates $\left(\mathrm{nC}_{7}\right.$ or $\mathrm{nC}_{10}$ in solution with iso-octane) on combustion characteristics in a $\mathrm{HCCl}$ engine. Simulations were conducted using a new detailed chemical kinetic model developed by merging the MD mechanism proposed by Herbinet et al. [142] with reduced mechanisms for the hydrocarbon fuels [237] and [238], whereas the $\mathrm{HCCl}$ experiments were modeled using a zero-dimensional single-zone reactor model of CHEMKIN [158]. After a successful validation model against the shock tube data for each 
pure component $\left(\mathrm{nC}_{7}, \mathrm{nC}_{10}\right.$, iso-octane, and $\mathrm{MD}$ ), the authors analyzed $\mathrm{HCCl}$ engine simulations and observed that combustion phasing (evaluated using CA50, the crank angle for $50 \%$ heat release) was increased up to an additional $10 \%$ by volume of MD into each petrodiesel surrogate, whereas larger additions of MD (beyond $12.5 \%$ by volume) showed almost negligible effects. The author highlighted that further investigation was needed to understand this behavior of MD reactivity.

Very recently, two modeling studies were published in the literature. Diévart et al. [214] developed a detailed kinetic model for the oxidation of MD. The model developed in this study was designed from the original oxidation framework of MD proposed by Herbinet et al. [142]. It was successfully tested against data from the literature $[153,188,189,192,193]$ and was compared with other models from the literature [142,153] showing similar performances. As for MB, Grana et al. [147] developed a lumped kinetic model for the oxidation and pyrolysis of MD. Comparisons were performed using experimental data from the literature obtained in a wide range of conditions: temperature ranging from 500 to more than $2000 \mathrm{~K}$, pressures up to 16 bar, and equivalent ratios from lean to pyrolysis conditions $[153,177,190,192,193]$. The validation showed that, despite the simplifications due to the lumping strategy, the model was able to reproduce the experimental measurements in pyrolysis as well as in an oxidation environment, in both the low-temperature regime and in flame conditions.

\subsubsection{Oxidation of methyl-5- and methyl-9-decenoate}

Although the Herbinet et al. model [142] was determined to be a powerful tool for predicting biodiesel combustion, methyl decanoate (MD), which the model was based on, has no double bonds, unlike most actual biodiesel components. Therefore, to capture the impact of this chemical specificity on biodiesel reactivity and further refine the kinetic model, the same authors [143] investigated the oxidation of two $\mathrm{C}_{10}$-methyl esters with a double bond located at different positions on the aliphatic main chain: methyl-5-decenoate (MD5EN) and methyl-9-decenoate (MD9EN). Because of the similar molecular structures of the selected biodiesel surrogates, the two detailed chemical kinetic sub-mechanisms for the oxidation of MD5EN and MD9EN were generated from the previous MD oxidation models by adding the low- and high-temperature chemistry specific to the unsaturated species (presence of double bonds, vinylic and allylic $\mathrm{H}$-atoms). Both models for MD5EN and MD9EN oxidation were compared with rapeseed oil methyl ester experiments in a JSR [197]. It was observed that MD9EN performed better than MD5EN in reproducing the reactivity and mole fraction profiles of the major species. The computed ignition delay times for MD5EN and MD9EN were also compared, which showed that MD9EN was more reactive than MD5EN, particularly in the NTC region, and led to the conclusion that MD9EN would be a better biodiesel surrogate than MD5EN. The authors attributed the lower reactivity of MD5EN to more difficult isomerization reactions of peroxy radicals $\mathrm{RO}_{2}$ over the double bond because of its location in the middle of the aliphatic main chain. Thus, the authors [143] highlighted that it is actually the length of the continuously saturated carbon chain in the reactant that determines its reactivity because this chain sets the range of possible $\mathrm{RO}_{2}$ isomerization reactions.

To obtain a detailed chemical kinetic mechanism that would be more representative of biodiesel fuels, Herbinet et al. [143] combined the three models of oxidation related to MD, MD9EN, and $\mathrm{n}$-heptane in order to match the $\mathrm{C} / \mathrm{O} / \mathrm{H}$ content in actual biodiesels. The resulting "blend surrogate model" was used to simulate the rapeseed oil methyl ester experiments conducted in a JSR by Dagaut et al. [197]. The "blend surrogate model" performed slightly better than the model for MD9EN by reproducing the experimental mole fraction profiles of most species with good agreement. These results led the authors to recommend this "blend surrogate model" for simulating the combustion of biodiesel fuels regardless of their origin by adjusting the mole fractions of the three components in the surrogate blend. 


\subsubsection{Pyrolysis of methyl decanoate}

As previously mentioned for MB pyrolysis (Section 3.4.2), thermal decomposition and oxidation studies are complementary and necessary to perform the validation of detailed kinetic models. This knowledge is especially important for biodiesel surrogates because the first reactions of diesel fuels in the combustion chamber are similar to a pyrolysis process, which lead to a high amount of unsaturated products and soot that are later oxidized in the flame front. Hence, Herbinet et al. [190] performed an experimental and modeling study of MD thermal decomposition in a JSR close to atmospheric pressure at temperatures ranging from 773 to $1123 \mathrm{~K}$ for various residence times (Table 4). In addition to $\mathrm{H}_{2}, \mathrm{CO}_{2}$, and small hydrocarbons from $\mathrm{C}_{1}-\mathrm{C}_{3}$, the main reaction products were 1-olefins (from 1-butene to 1-nonene) and unsaturated esters with one double bond at the extremity of the aliphatic main chain (from methyl-2-propenoate to methyl-8-nonenoate). In addition, the formation of polyunsaturated species (1,3-butadiene, 1,3-cyclopentdiene, benzene, toluene, indene, and naphthalene) was observed at the highest temperatures. Comparison of pyrolysis with an nalkane of similar size to MD ( $\mathrm{n}$-dodecane) led the authors to observe that both molecules had similar reactivity (Fig. 11); the $\mathrm{n}$-alkane produced more olefins while the ester yielded unsaturated oxygenated compounds. The detailed kinetic model for MD thermal decomposition (Table 5), which was generated from the latest version of EXGAS software [152,153], provided a good prediction of the JSR experimental data and showed through kinetic analysis that the retro-ene reactions (which were added to the secondary mechanism specifically for this work) play an important role in the consumption of 1-olefins and unsaturated methyl esters, particularly in low-reactivity conditions. Nevertheless, as retro-ene reactions consist of intra-molecular decomposition reactions into propene and smaller unsaturated esters through a concerted mechanism involving a six-membered cyclic transition state and transfer of an $\mathrm{H}$-atom at the $\gamma$-position of the $\mathrm{C}=\mathrm{C}$ double bond (Fig. 12), only species larger than methyl-4-pentenoate are concerned by this class of molecular reactions. Consequently, small unsaturated esters (such as methyl-4-pentenaote and methyl-3-butenoate) were overpredicted (by a factor of approximately 2), indicating that a pure radical mechanism is not sufficient for these species specifically and should be completed with molecular reactions.
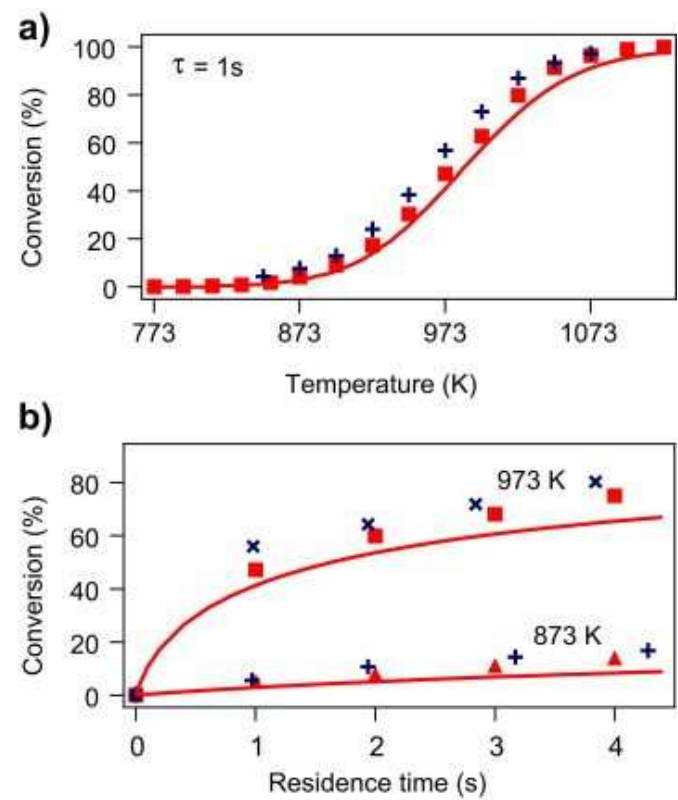

Figure 11. Conversion of methyl decanoate and $n$-dodecane pyrolysis in JSR versus temperature (a) and residence time (b). ( $\boldsymbol{\square}$ and $\boldsymbol{\Delta}$ : methyl decanoate experiments; $\times$ and $+: \mathrm{n}$-dodecane experiments; line: methyl decanoate simulations). Inlet mole fractions of $n$-dodecane and methyl decanoate are 0.02 and 0.0218 , respectively [190]. 


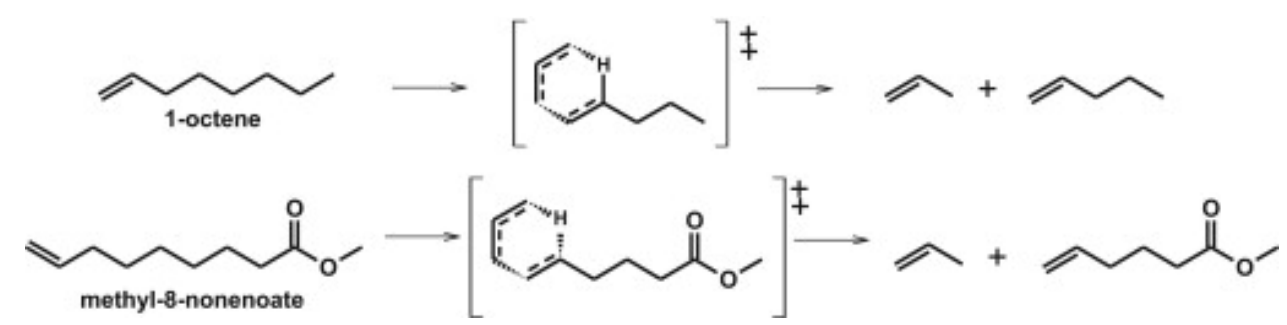

Figure 12. Retro-ene reactions illustrated with 1-octene and methyl-8-nonenoate [190].

\subsubsection{Oxidation of actual biodiesel molecules}

As mentioned previously (Table 1), methyl myristate (MM), methyl palmitate (MP), methyl stearate (MST), methyl oleate (MO), methyl linoleate (MLO), and methyl linolenate (MLN) are components of methyl biodiesels derived from vegetable oils. The first three species are saturated $C_{n}$-methyl esters with $n$ equal to $14(\mathrm{MM}), 16(\mathrm{MP})$, and 18 (MST), whereas the last three species are unsaturated $\mathrm{C}_{18}$-methyl esters with one (MO), two (MLO), and three (MLN) double bonds $(-\mathrm{CH}=\mathrm{CH}-)$. MP, $\mathrm{MO}$, MLO and MLN are found in varying amounts in the methyl esters of most vegetable oils, while MM is rather in trace amounts (Table 1 ).

Among these large fatty acid methyl esters, MP and MO were first investigated experimentally in mixtures with $n$-decane $[194,195]$ to simulate biodiesel fuel oxidation behavior when blended with petrodiesel. For MP, comparisons were also conducted with $n$-decane/n-hexadecane as a blended surrogate for petrodiesel fuels; indeed, $n$-hexadecane (selected as reference fuel for the CN test, Section 2.3.1.2) and the MP aliphatic main chain have the same number of carbon atoms. For all surrogate blends, experiments were performed in a JSR over a wide temperature range covering low and high temperatures $(550-1100 \mathrm{~K})$ at quasi atmospheric pressure and stoichiometric conditions (Table 4). Numerous reaction products were identified and quantified $[194,195]$, as will be observed in the following sections.

On the basis of these measurements, detailed kinetic models of large methyl ester oxidation were later developed and validated (Table 5). Herbinet et al. [213] proposed oxidation mechanisms for saturated esters from methyl decanoate up to methyl stearate using the automatic generator in EXGAS software [152,153]. Naik et al. [144] and Westbrook et al. [145] proposed oxidation mechanisms for saturated and unsaturated C18-methyl esters using iterative generation from previous mechanisms developed for methyl decanoate [142] and methyl decenoates [143] that were appropriately extended to the larger alkyl chain esters (MST, MO, MLO, and MLN) with additional $\mathrm{C}=\mathrm{C}$ bonds (two for MLO and three for MLN).

Comparison of the observed results highlighted the similarities and differences in the oxidation of large n-alkanes and methyl esters as well as unsaturated and saturated methyl esters of similar size. On the basis of these observations, the researchers next determined the optimal surrogate blend and detailed kinetic models in terms of size and performance to simulate and predict reliably the methyl biodiesel combustion properties. These issues are briefly described in the following sections.

\subsubsection{Methyl palmitate versus $n$-hexadecane [194]}

$\mathrm{MP}$ and $\mathrm{n}$-hexadecane $\left(\mathrm{nC}_{16}\right)$ showed similar reactivity and the same NTC behavior over the temperature range studied, which confirmed previously observed results by Herbinet et al. [142] for $\mathrm{MD}$ and $\mathrm{n}$-decane. Furthermore, most of the observed species were formed with two surrogate blends, $\mathrm{n}$-decane/MP and $\mathrm{n}$-decane/ $\mathrm{nC}_{16}$. These common products are small oxygenated species (carbon oxides, methanol, and acetaldehyde), small hydrocarbons (methane, acetylene, ethylene, and ethane), and 1-olefins. The products observed depended on the temperature of oxidation. At 
high temperatures, the products were monounsaturated esters with the double bond at the extremity of the aliphatic main chain for MP (type A molecules, Fig. 13) and 1-olefins for $\mathrm{nC}_{16}$. At low temperatures, the specific products for MP were methyl esters with a cyclic ether group located on the aliphatic main chain and a 5-membered cyclic ether ring, including the ester group, branched to a long-aliphatic main chain (type $\mathrm{B}$ and $\mathrm{C}$ molecules, respectively, Fig. 13); regarding $\mathrm{nC}_{16}$, the specific products were 5 -membered cyclic ether rings and ketones branched on an aliphatic chain with 16 carbon atoms. The first class of compounds that occurred at high temperatures were also observed by Szybist et al. $[186,187]$ for MD, whereas for the second class of compounds that occurred at low temperatures, Szybist et al. observed oxo-alkanoic acid methyl esters and cyclic esters, probably because of the shorter aliphatic main chain of the ester MD (Table 11).

A

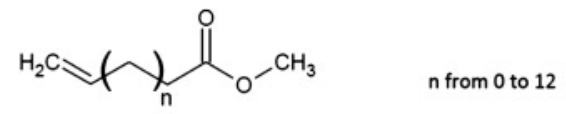

B

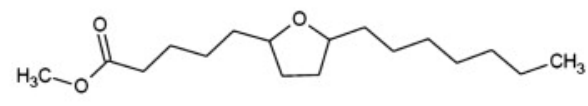

C

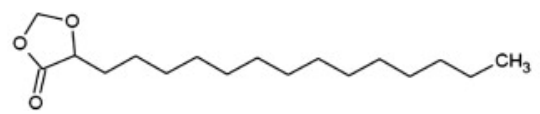

Figure 13. Samples of molecules observed by Hakka et al. [194] during oxidation of the ndecane/methyl palmitate blend (species A observed at high temperatures, species $B$ and $C$ at low temperatures).

\subsubsection{Methyl palmitate versus methyl oleate [195]}

Bax et al. reported that both molecules (MP and MO) exhibited similar reactivity with the NTC zone, occurring at approximately $650-750 \mathrm{~K}$. Nevertheless, because of the presence of the double bond in the middle of the aliphatic main chain for $\mathrm{MO}$, specific reactivity features and reaction products were noted by the authors. First, MO appeared to be slightly less reactive than MP in the low-temperature range (below $750 \mathrm{~K}$ ) despite two additional carbon atoms relative to MP, whereas the opposite trend was observed beyond the NTC region. The authors attributed this behavior to the presence of the double bond, which disfavored low-temperature chain-branching reactions, mainly the isomerization of peroxy radicals $\mathrm{RO}_{2}$ into hydroperoxy radicals $\mathrm{QOOH}$ (reaction 4, Fig. 4). Concerning the reaction products specific to $\mathrm{MO}$ oxidation, dienes and esters with two double bonds (resulting from decomposition by $\beta$-scission of the two allylic radicals from $\mathrm{H}$-abstraction of $\mathrm{MO}$, Fig. 14) were observed at high temperatures (approximately $800 \mathrm{~K}$ ). However, at low and intermediate temperatures (approximately 550-650 K), oxygen-containing compounds were observed, namely aldehydes conjugated with one double bond (Fig. 15a) and $\mathrm{C}_{18}$-methyl esters with either one 3-membered cyclic ether functional group (oxirane esters, Fig. 15b) or one ketone functional group (oxo-esters, Fig. 15c) branched on the aliphatic main chain. These species were hypothesized to come from a combination of $\mathrm{HO}_{2}$ radicals with the resonance stabilized radicals from $\mathrm{MO}$ (conjugated aldehydes) or from the addition of $\mathrm{HO}_{2}$ or $\mathrm{HO}$ to the double bond in $\mathrm{MO}$ (leading to oxirane or oxo-esters, respectively). The authors concluded their work by highlighting that, based on recent investigations related to large methyl ester modeling [142,143], a detailed chemical kinetic model for MO generated by EXGAS software [152,153] would contain more than 50,000 reactions and 6000 species, making its application difficult. 


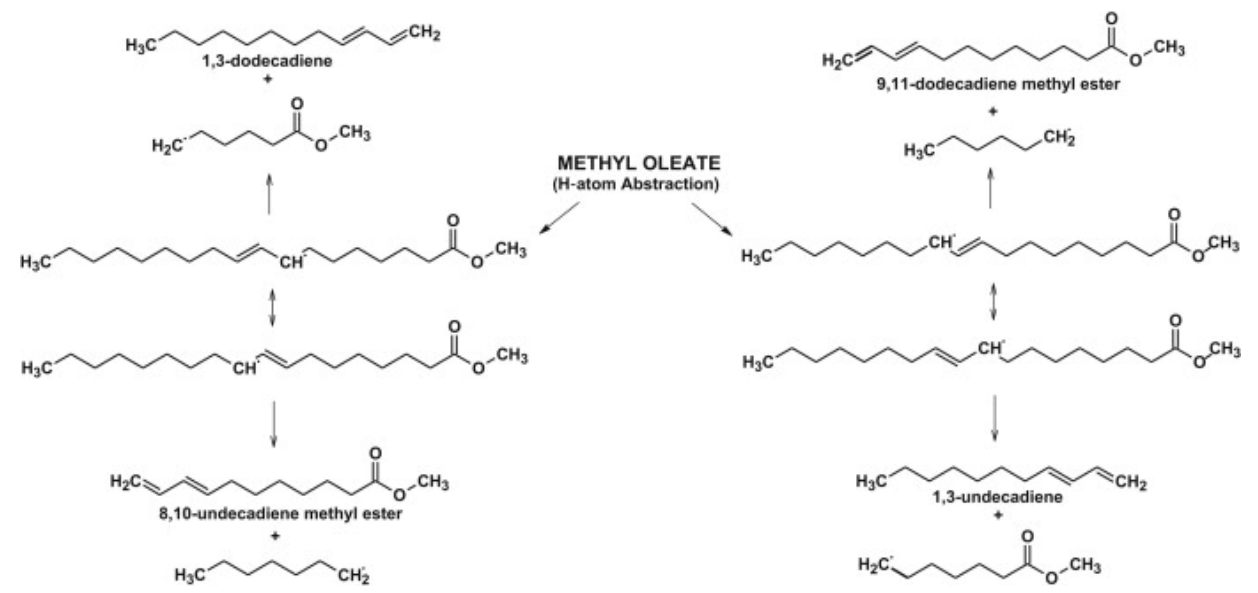

Figure 14. Illustration of dienes and esters with two double bonds formed during high-temperature $\mathrm{n}$-decane/methyl oleate oxidation (species observed around $800 \mathrm{~K}$ and due to decomposition by $\beta$-scission of the two allylic radicals resulting from $\mathrm{H}$-abstraction of methyl oleate and stabilization by resonance) [195].

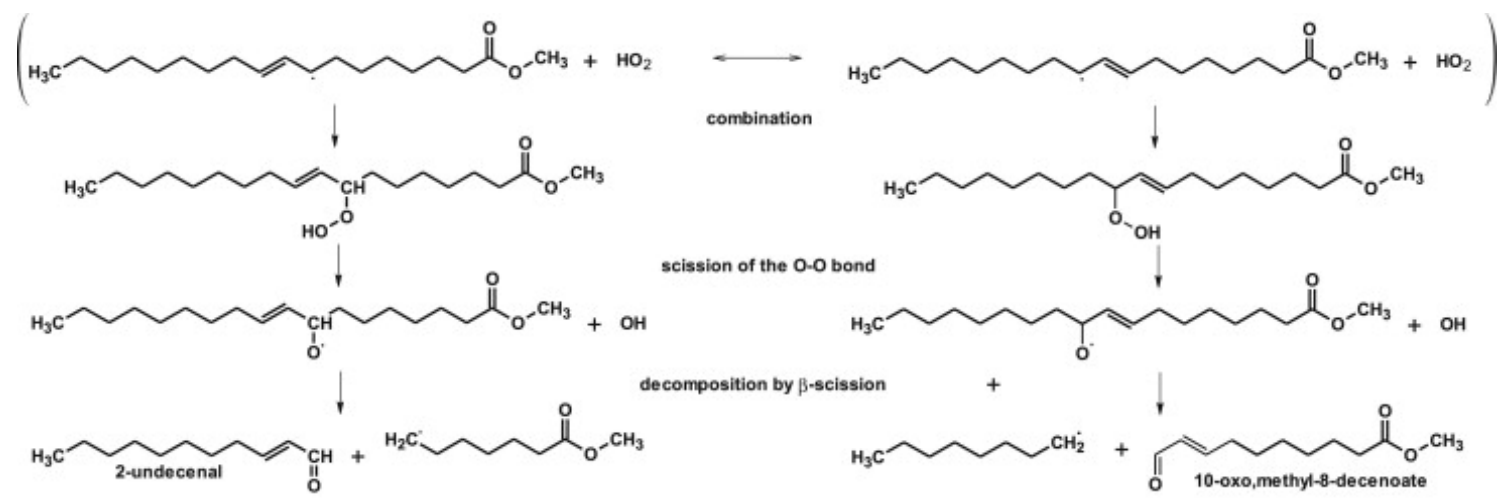

(a)

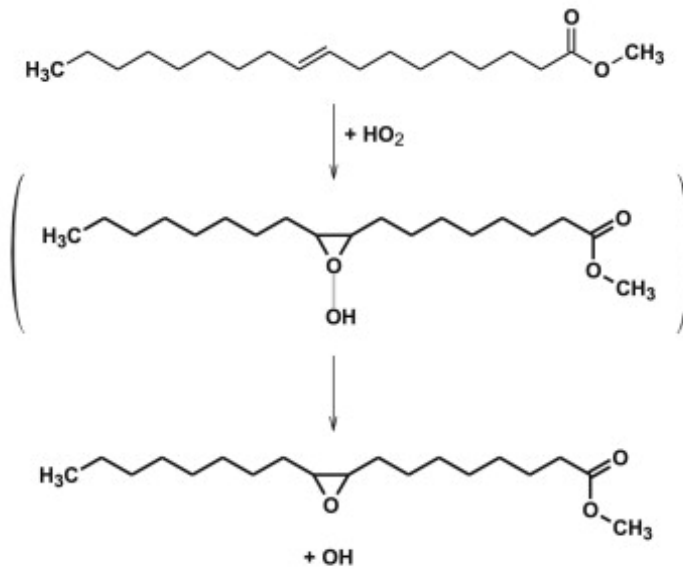

(b)

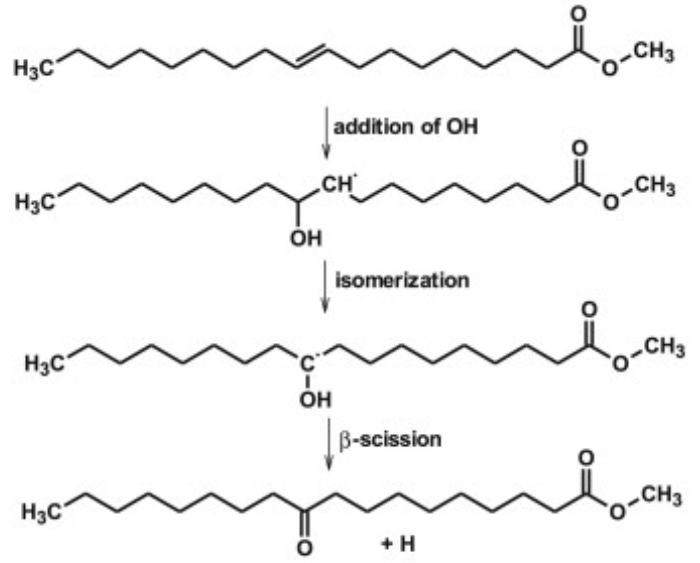

(c)

Figure 15. Illustration of oxygen-containing compounds formed during low-temperature n-decane/methyl oleate oxidation (550-650 K) and their possible channels of formation. (a) Aldehydes conjugated with one double bond; (b) $\mathrm{C}_{18}$-methyl esters with one 3-member ring cyclic ether functional group (oxirane esters illustrated here by 2-octyl, 3-(methyl octanoate)-oxirane); (c) $\mathrm{C}_{18}$-methyl esters with one ketone functional group (oxo-esters illustrated here by 10-oxo, methyl octadecanoate) [195]. 


\subsubsection{Methyl stearate versus methyl oleate, methyl linoleate, and methyl linoleate $[144,145]$}

The major refinements of the detailed chemical kinetic reaction mechanisms proposed by Naik et al. [144] (for MST and MO) and Westbrook et al. [145] (for the whole of these actual biodiesel components) in comparison with the work by Herbinet et al. [143] for the methyl decenoates (MD5EN and MD9EN) consist of three actions. The first (i) is to include accurate site-specific reaction rates for MO, MLO, and MLN as these species are products of oxidation of MST, MO, and MLO, respectively, and the major components of RME or SME (Table 1). However, olefins, di-olefins, and tri-olefins were treated as lumped species in the same way that the authors treated alkene and alkenyl radicals (reaction classes $6-9$, Table 6 ) in past mechanisms for saturated hydrocarbon fuels $[207,217]$ and the saturated methyl ester MD [142]. For the lumped olefins, di-olefins, and tri-olefins with the methyl ester group included, each species is assumed to react through $\mathrm{H}$-atom abstraction reactions (with $\mathrm{H}, \mathrm{O}, \mathrm{OH}, \mathrm{HO}_{2}, \mathrm{CH}_{3}, \mathrm{CH}_{3} \mathrm{O}, \mathrm{CH}_{3} \mathrm{O}_{2}, \mathrm{C}_{2} \mathrm{H}_{3}, \mathrm{C}_{2} \mathrm{H}_{5}$, and $\mathrm{O}_{2}$ ), which produces a single lumped radical that is assumed to decompose to smaller species and eventually to small, usually unsaturated radicals, in the $\mathrm{C}_{1}-\mathrm{C}_{4}$ core kinetic mechanism. The second action (ii) is to include $\mathrm{MO}$ in MST oxidation mechanism as a reactant (with reaction classes 1-5 in Table 6 but with some $\mathrm{H}$-abstractions from allylic and vinylic sites as well as isomerization of the MO radicals, since both reaction types depend on the location of the double bond). Finally, the third action (iii) is to assume that $\mathrm{RO}_{2}$ and $\mathrm{O}_{2} \mathrm{QOOH}$ isomerization does not proceed in the low-temperature mechanism if a double bond is contained within the transition-state ring for the reaction (while in the mechanism by Herbinet et al. [143] for methyl decenoates, the double bond was assumed to contribute an additional $15 \mathrm{kcal} \mathrm{mol}^{-1}$ to the energy barrier for these isomerization reactions). Action (ii) used the work by Naik et al. [144] related to $\mathrm{MO}$ oxidation that noted two very important features. First, radical addition to the $\mathrm{C}=\mathrm{C}$ double bond contributes slightly to the overall rate of reaction; however, most of the MO consumption occurs through $\mathrm{H}$-atom abstraction. Second, the rate of $\mathrm{H}$-atom abstraction from the site adjacent to the carbonyl group is higher than abstractions from the secondary $\mathrm{C}-\mathrm{H}$ bond sites; however, abstraction from the allylic sites is even faster, whereas abstraction from the vinylic sites is much too slow to be important.

Model validations for MP and MO [145] compared with the JSR experiments [194,195] showed good agreement over the entire temperature range for the fuel molecules and main products. These model validations also confirmed two specific features observed experimentally: (i) the formation of the species typical of fuel molecule oxidation (shown in Fig. 13 for MP and in Figs. 14 and 15 for MO) and (ii) the occurrence of a low-temperature reaction regime as well as higher temperature reactivity for the major reaction intermediates (1-olefins and 1-olefin methyl esters in MP oxidation). Recently, data computed with the model proposed by Westbrook et al. [145] were compared with ignition delay times of MO and MLO measured behind reflected-shock waves using an aerosol shock tube [196]. The comparison showed an under-prediction of the reactivity by about $50 \%$. The thermochemistry of the fuels was refined resulting in significant performance improvements.

Differences in the combustion properties between each methyl ester (MO, MLO, and MLN) were noted by Naik et al. [144] and Westbrook et al. [145] by performing simulations of oxidation in a JSR (Fig. 16) and a ST environment (Fig. 17) for each of the species individually considered. Concerning the JSR simulations (Fig. 16), the two saturated methyl esters, MP and MST, showed similar behavior with a pronounced low-temperature reaction zone and a small difference at approximately $750 \mathrm{~K}$ where MP showed slightly less conversion. However, the unsaturated methyl esters with one (MO), two (MLO) or three (MLN) CC double bonds showed significantly different behavior, particularly MLO and MLN. MO presented decreased low-temperature reactivity compared to its homologous saturated methyl ester MST but more fuel conversion for temperatures above $700 \mathrm{~K}$, whereas MLO presented relatively little low-temperature reactivity with a small NTC region at $700 \mathrm{~K}$, and MLN presented further decreased low-temperature reactivity with no NTC behavior. Concerning autoignition simulations (Fig. 17), all five methyl esters had nearly equal ignition delay times at high 
temperatures, which differed primarily at temperatures below $900 \mathrm{~K}$, including the NTC region. While in this region, the two saturated methyl esters, MP and MST, showed nearly the same behavior, the ignition delay times of the unsaturated methyl esters were determined to decrease with the number of $\mathrm{C}=\mathrm{C}$ double bonds. As MST, MO, MLO, and MLN have the same size and structure, except for the number of $\mathrm{CC}$ double bonds in the aliphatic main chain, differences in oxidation behavior observed in the low-temperature regime must be because of the occurrence of this bond, which also induces a decrease in CN. As highlighted by Westbrook et al. [145], the whole of these observations show a correlation between the chemical structure of the fuel molecule, cetane number, ignition delay, and low-temperature reactivity (only mentioned for the first three properties in Section 2.3.1.2). The authors conclude that fuels with greater amounts of low-temperature reactivity and heat release have shorter ignition delays and ignite earlier as well as have higher cetane numbers than fuels with decreased low-temperature reactivity. Naik et al. [144] concluded that this phenomenon was due to the $\mathrm{C}=\mathrm{C}$ double bond that inhibits $\mathrm{RO}_{2}$ and $\mathrm{O}_{2} \mathrm{QOOH}$ isomerization when the $\mathrm{C}=\mathrm{C}$ double bond is embedded in the transition-state ring, thereby reducing the overall rate of low-temperature chainbranching. These features will be observed again in the following section.

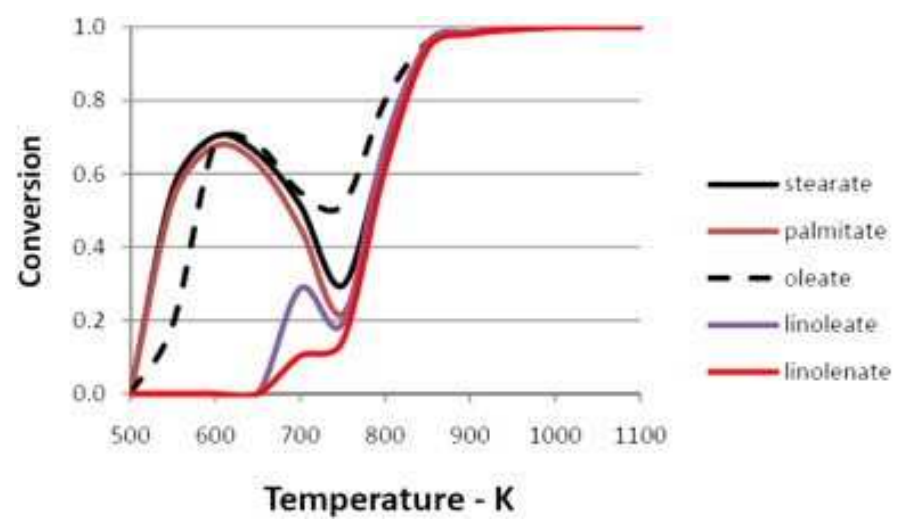

Figure 16. JSR simulations carried out for five actual biodiesel components: methyl palmitate, methyl stearate, methyl oleate, methyl linoleate, and methyl linolenate. Operating conditions adopted for the simulations: stoichiometric fuel/oxygen, $0.2 \%$ fuel, with helium diluents, $\mathrm{P}=1 \mathrm{~atm}$, and residence time $1.5 \mathrm{~s}$ [145].

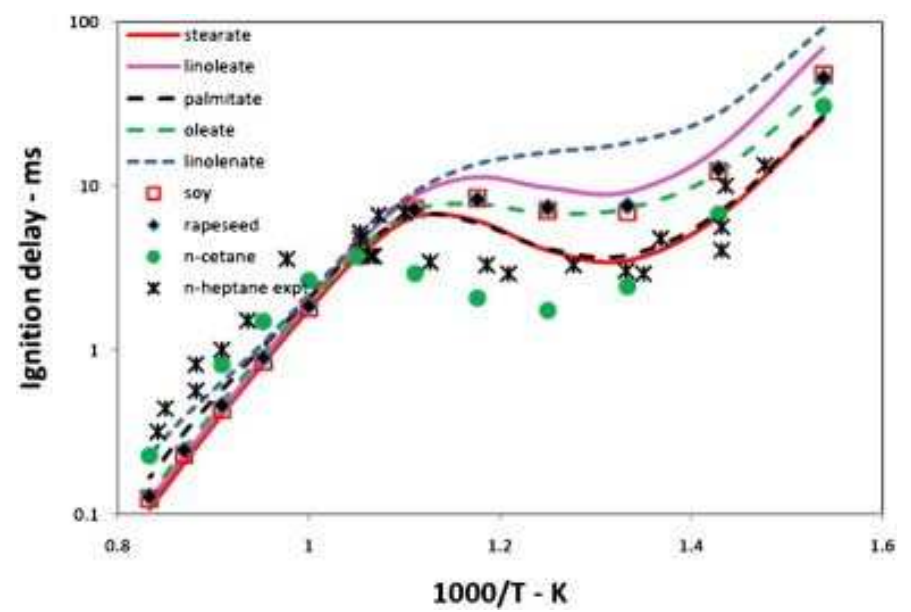

Figure 17. Ignition delay times for stoichiometric fuel/air mixtures in a reflected-shock tube environment at 13.5 bar initial pressure. Except asterisks showing experimental results for $\mathrm{n}$-heptane/air, other results are kinetic model predictions: lines depict predicted values for each methyl ester fuel, circles for $n$-cetane (i.e. $n$-hexadecane), squares and diamonds for the surrogates SME and RME (Table 13) respectively [145]. 


\subsubsection{Determination of the optimal kinetic model in terms of size and performance for a reliable prediction of methyl biodiesel combustion properties}

With this objective, Herbinet et al. [213] developed detailed kinetic mechanisms for saturated esters from methyl decane (complementary to the work by Glaude et al. [153]) up to methyl stearate (from $\mathrm{C}_{10^{-}}$to $\mathrm{C}_{1} 6$-methyl esters). These mechanisms, which were automatically generated using the latest version of the EXGAS software [152,153], include the reactions specific to the chemistry of large saturated esters and a single set of kinetic parameters. An exhaustive description of these refinements (and the general rules that led to them) is presented in the work by Glaude et al. [153]; therefore, the main features have just been briefly mentioned here.

The changes concern both the primary mechanism (where the only molecular reactants considered are the initial organic compounds and oxygen) and the lumped secondary mechanism (consuming the molecular products of the primary mechanism). Regarding the primary mechanism, the changes affect the activation energies of four main classes of reactions: (i) unimolecular initiations, which involve the breaking of the $\mathrm{C}-\mathrm{C}$ bond located in the $\alpha$ - and $\beta$-position from the ester function (values were updated according to El-Nahas et al. ab initio estimations [225]); (ii) radical oxidations (yielding an unsaturated molecule and $\mathrm{HO}_{2}$ ) and $\mathrm{H}$-abstraction from the carbon adjacent to the ester function (both classes of reactions were considered as if they concerned a tertiary $\mathrm{H}$-atom); (iii) radical intramolecular isomerization reactions involving cyclic transition states with the embedded ester group; and (iv) radical decomposition by $\beta$-scission involving the ester group for which quantum calculations were performed. Regarding the lumped secondary mechanism, the changes concern the new rules that had to be implemented for the consumption of the species formed specifically from ester oxidation in the primary mechanism. These new rules were based on the general idea promoting the formation of radicals via reactions that are already included in the primary mechanism (similar to Ref. [150] for alkanes).

The performance of the MD model has been previously demonstrated by Glaude et al. [153] and model validation was achieved by Herbinet et al. [213] against the JSR experiments related to the MP species [194]. Very good predictions of the MP fuel reactivity and mole fraction profiles of most reaction products have been observed. In addition, as previously described for the MD species, predictions obtained for MP fuel were of the same level of agreement compared to experiments as those performed by Westbrook et al. [145], although both models are based on the same general departure (Section 3.2) but different generation approaches (Section 3.1.1). In addition to model validation, Herbinet et al. [213] compared the combustion properties of the selected methyl esters by individually simulating JSR oxidation of each fuel (Fig. 18). Moreover, to focus the comparison onto the molecular structure of the fuels, the inlet mole fractions were calculated to maintain a constant carbon content of each reacting mixture. All selected methyl esters exhibited similar conversion curves with an S shape because of the NTC (observed at $780 \mathrm{~K}, \mathrm{Fig}$. 18). In this region, the reactivity of the methyl esters increased when the length of the aliphatic main chains increased (from $\mathrm{C}_{10}$ - to $\mathrm{C}_{18}$-methyl ester). Furthermore, the reactivity of $\mathrm{n}$-hexadecane (also simulated by Herbinet et al. [213]) appeared to align the reactivity of the methyl esters with however a lower conversion in the NTC region and a slightly larger conversion at lower temperatures (below $750 \mathrm{~K}$ ). Hence, Herbinet et al. [213] concluded that large n-alkanes, such as $n$-hexadecane, could be good surrogates for reproducing the overall reactivity of large methyl esters (as confirmed by Dagaut et al. [197] in next Section 3.4.7) with an important gain in computation time. Nevertheless, n-alkanes could not account for the formation of specific products, such as saturated esters or cyclic ethers with an ester function. Nevertheless, a mid-sized methyl ester, such as methyl decanoate, predicts the reactivity and molar fractions of most species fairly well with a substantial decrease in computational time and would be a good compromise as a biodiesel surrogate. Also, actual FAME components involve species with CC double bonds that induce specific changes in the combustion properties, as discussed previously [145]. Although Bax et al. [195] experimentally observed with MO fuel that the presence 
of a single $\mathrm{C}=\mathrm{C}$ double bond in the middle of the aliphatic main chain had little effect on the reactivity of large molecules, this feature should not be observed for FAME with more embedded $\mathrm{C}=\mathrm{C}$ double bonds, such as MLO (and MLN according to Westbrook et al. [145]). Therefore, one might conclude that an optimal biodiesel surrogate and optimal kinetic model would be selected depending on the objectives and applications.

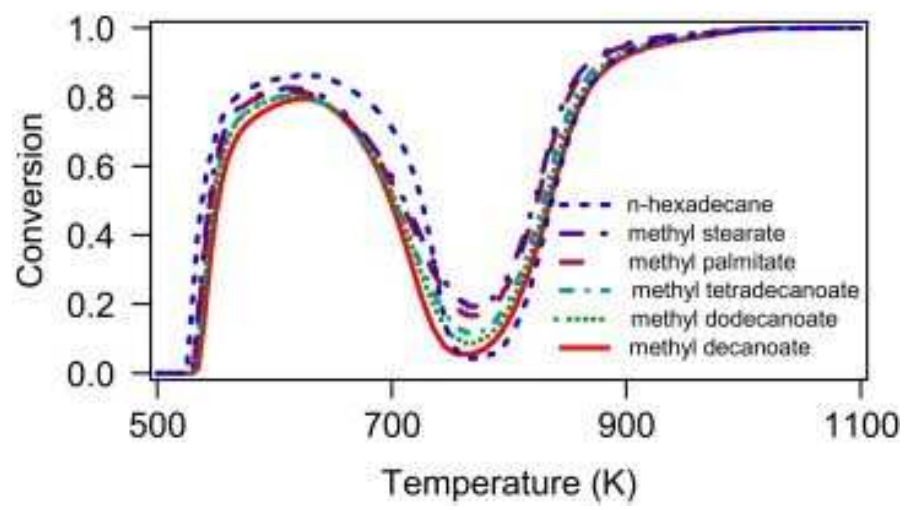

Figure 18. Comparison of the computed conversion of large saturated esters from methyl decanoate up to methyl stearate and $\mathrm{n}$-hexadecane in a jet-stirred reactor [213].

\subsubsection{Oxidation of rapeseed and soybean oil methyl esters}

Pedersen et al. [239] performed a qualitative study of the species formed during the oxidation of rapeseed oil and RME in a stainless steel tubular reactor at $823 \mathrm{~K}$. GC-MS analysis of the two fuel gaseous emissions led the authors to conclude that rapeseed oil and RME react in a similar way during oxidation regarding the formation of hydrocarbons (1-alkenes, dienes, and benzene). Nevertheless, the authors observed that rapeseed oil oxidation produced high amounts of acrolein and other aldehydes, whereas RME oxidation produced significant amounts of methyl acrylate (methyl-2-propenoate) and other unsaturated esters, including methyl-3-butenoate, methyl-5hexenoate, and methyl-6-heptenoate. The formation of methyl-4-pentenoate was not observed.

Quantitative investigation of RME oxidation was conducted in a JSR for the first time by Dagaut et al. [197]. The work developed by these authors was extensively used by researchers performing kinetic modeling investigations to validate their detailed chemical kinetic model on actual biodiesel oxidation experiments $[142,143]$. Details related to this significant experimental work are presented here. Experiments by Dagaut et al. [197] were conducted in dilute conditions over a wide range of temperatures $(800-1400 \mathrm{~K})$, under low to moderate pressures (1-10 atm), and for various equivalence ratios and residence times (Table 4). Analysis of the mole fractions for measured species led the authors to observe a strong similarity between oxidation of RME and oxidation of large $\mathrm{n}$-alkanes. The experimental species profiles were compared with computed mole fractions from a mechanism previously developed for the oxidation of $n$-hexadecane $\left(\mathrm{nC}_{16}\right)$, which consisted of 225 species and 1841 reversible reactions [215] (Table 5). The agreement was shown to be satisfactory, and $n$-hexadecane appeared to be a good surrogate for RME under the investigated conditions. However, as it can be expected at this stage of the review, the $\mathrm{nC}_{16}$ mechanism was unable to predict the early production of $\mathrm{CO}_{2}$ that was observed in the experiments. According to assumptions reported by Szybist et al. $[186,187]$, Dagaut et al. [197] suggested reactions responsible for this phenomenon but without giving further mechanistic considerations

$\mathrm{C}_{\mathrm{n}} \mathrm{H}_{\mathrm{m}}(\mathrm{CO}) \mathrm{OCH}_{3} \rightarrow \mathrm{C}_{\mathrm{n}} \mathrm{H}_{\mathrm{m}}(\mathrm{CO}) \mathrm{O}+\mathrm{CH}_{3}$

$\mathrm{C}_{\mathrm{n}} \mathrm{H}_{\mathrm{m}}(\mathrm{CO}) \mathrm{O} \rightarrow \mathrm{C}_{\mathrm{n}-1} \mathrm{H}_{\mathrm{m}-2} \mathrm{CH}_{2}+\mathrm{CO}_{2}$ 
Later, Dagaut and Gaï [200] investigated the oxidation of a blend of RME and kerosene Jet-A1 (20/80 $\mathrm{mol} \mathrm{mol}^{-1}$ ) in a JSR. As far as the scope of this review with biodiesel fuels, only results related to RME oxidation will be reported. Experiments that were performed over a wide temperature range (740$1200 \mathrm{~K}$ ) at $10 \mathrm{~atm}$ for various equivalence ratios and a constant residence time (Table 4) revealed the formation of monounsaturated methyl esters with a $\mathrm{C}=\mathrm{C}$ double bond at the extremity of the aliphatic main chain (methyl-2-propenoate, methyl-3-butenoate, methyl-4-pentenoate, and methyl5-hexenoate).

More recently, Golovitchev and Yang [216] developed an RME combustion model for internal combustion engine applications. By assigning methyl linoleate $\left(\mathrm{C}_{19} \mathrm{H}_{34} \mathrm{O}_{2}\right)$ as model molecule for RME (although methyl oleate might be a better choice on the basis of the RME profile, Table 1), the authors based their RME combustion mechanism on the global decomposition reaction

$\mathrm{C}_{19} \mathrm{H}_{34} \mathrm{O}_{2}+0.5 \mathrm{O}_{2} \rightarrow \mathrm{C}_{5} \mathrm{H}_{10} \mathrm{O}_{2}+\mathrm{C}_{7} \mathrm{H}_{16}+\mathrm{C}_{7} \mathrm{H}_{8} \mathrm{O}$

leading to products for which detailed oxidation sub-mechanisms were available in the literature. Species $\mathrm{C}_{5} \mathrm{H}_{10} \mathrm{O}_{2}$ representing $\mathrm{MB}$ was modeled with the Fisher et al. [208] mechanism, whereas $\mathrm{C}_{7} \mathrm{H}_{16}$ and $\mathrm{C}_{7} \mathrm{H}_{8} \mathrm{O}$ species, representing respectively $\mathrm{n}$-heptane and phenyl methyl ether, were modeled with the Golovitchev's mechanisms [240]. The resulting biodiesel surrogate blend model produced a detailed RME combustion mechanism that consisted of 309 species and 1472 reactions, including soot and NOx formation processes, and was successfully validated using shock tube ignition delay data related to RME surrogate components (MB, n-heptane, and phenyl methyl ether). Nevertheless, for modeling and simulating diesel engine (Volvo D12C) combustion (using the KIVA-3V code [241]), the authors reduced the detailed mechanism to 88 species participating in 363 reactions. The simulation results showed that RME combustion could be achieved with low soot and NO concentrations if moderate exhaust-gas recirculation loads, which induced a reduction in the combustion temperature, were used.

Recently, Westbrook et al. [145] applied the detailed chemical kinetic models they developed specifically for MST, MO (with the work by Naik et al. [144]), MLO, MLN, and MP to propose of a new reaction mechanism devoted to determine the differences in the combustion properties of two typical biodiesel fuels derived from soy and rapeseed oils (SME and RME, respectively). For this purpose, the authors combined the five methyl esters (MP, MST, MO, MLO, and MLN) into two different mixtures with composition approximating the SME and RME oils (Table 13) and performed oxidation simulation in a JSR (test 1 and test 2) and shock tube ignition (test 3) for these two 5-component biodiesel surrogates to validate the proposed reaction mechanism (Table 5). In addition, the same procedure can be extended to other methyl biodiesel fuels by specifying the convenient initial amounts of each of the five methyl ester components (MP, MST, MO, MLO, and MLN). Test 1. A comparison of the Westbrook et al. [145] reaction mechanism for the 5-component RME surrogate (Table 13) using the Dagaut et al. [197] experiments (related to RME oxidation in JSR) yields satisfactory overall agreement between the computed results and experimental values but with agreement for the profiles of the individual species varying from very good to marginal (particularly for $\mathrm{H} 2$ ). Test 2 and test 3 . The differences in combustion properties between RME and SME fuels were highlighted by simulating JSR oxidation (test 2) and intermediate shock tube ignition (test 3 ) at the same operating conditions as the conditions used to validate the five methyl esters individually (Section 3.4.6). From the computed conversions of RME and SME surrogates in a JSR (Fig. 19), two important features were noted by the authors. First, all five components appeared to react together as opposed to sequentially; however, they reacted at rather different overall rates for the RME and SME surrogates. Second, the net low-temperature reactivity of the RME surrogate was determined to be significantly greater than the SME surrogate because of the difference in CN (CN being approximately 54 for the RME fuel and 47 for the SME fuel). This last result is in agreement with the difference in composition of the two surrogates and differences in $\mathrm{CN}$ of their prevalent 
components (Table 13); the prevalent component in RME is MO, which is more reactive in the lowtemperature region than MLO, the prevalent component of SME. Nevertheless, nearly identical values of computed ignition delay times were obtained for the RME and SME surrogates; furthermore, these values were similar to the computed results for MO and MLO (Fig. 17). The differences in oxidation rates between RME and SME surrogates, which were greater in the atmospheric-pressure JSR simulations (Fig. 19) than in the high-pressure simulations related to shock tube ignition (Fig. 17), as well as the influence of pressure in the oxidation rates, were noted by the authors.

Table 13. Composition of the two surrogate biodiesel blended fuels adopted by Westbrook et al. [145] for representing each actual SME and RME. Also shown are the measured cetane numbers (CN) for each of the fuel components [145].

\begin{tabular}{cccc}
\hline Methyl esters & $\begin{array}{c}\text { Composition of the surrogate RME } \\
\text { fuel }\end{array}$ & $\begin{array}{c}\text { Composition of the surrogate SME } \\
\text { fuel }\end{array}$ & $\begin{array}{c}\text { Cetane } \\
\text { number (CN) }\end{array}$ \\
\hline Methyl palmitate (MP) & 4.3 & $6-10$ & 86 \\
Methyl stearate (MST) & 1.3 & $2-5$ & 101 \\
Methyl oleate (MO) & 59.9 & $20-30$ & 59 \\
$\quad \begin{array}{c}\text { Methyl linoleate } \\
\text { (MLO) }\end{array}$ & 21.1 & $50-60$ & 38 \\
$\begin{array}{c}\text { Methyl linolenate } \\
\text { (MLN) }\end{array}$ & 13.2 & $5-11$ & 23 \\
\hline
\end{tabular}
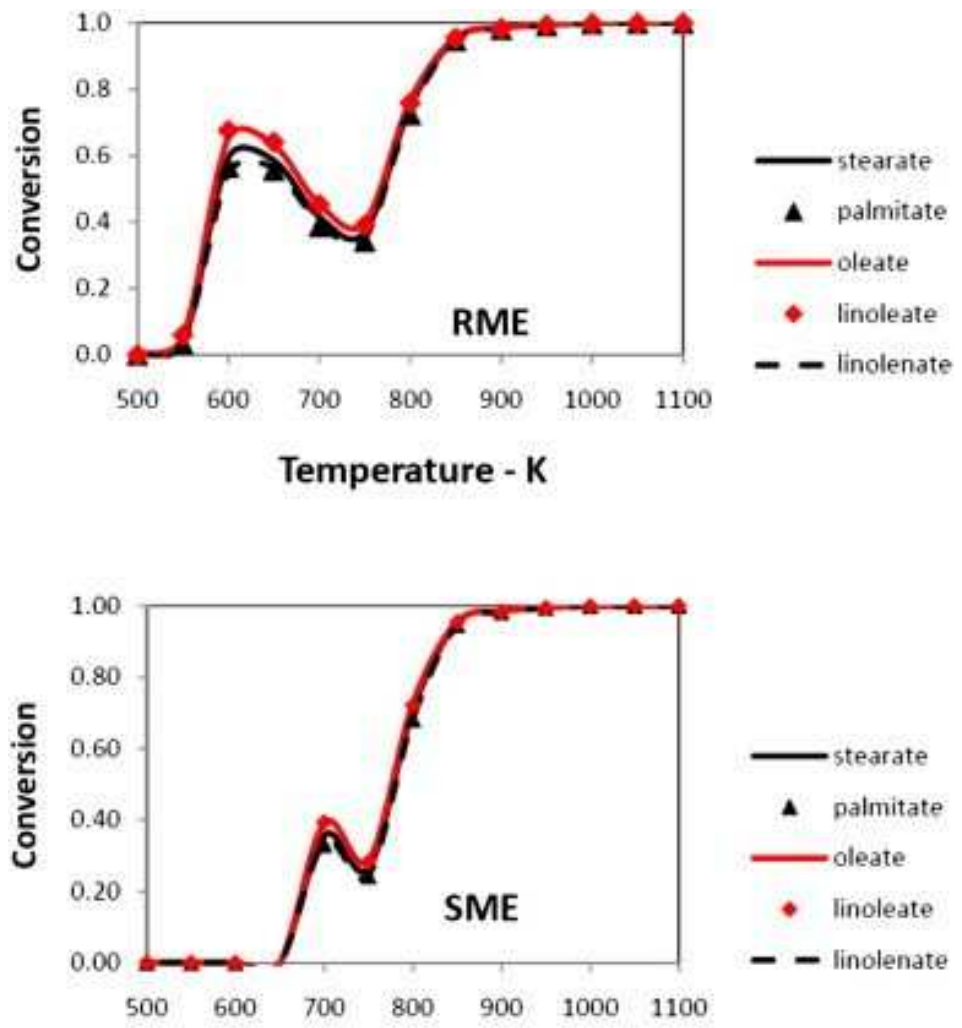

Temperature

Figure 19. Computed comparison between oxidation of RME and SME surrogates in a simulated JSR. Operating conditions: stoichiometric fuel/oxygen, $0.2 \%$ fuel, with helium diluents, $\mathrm{P}=1 \mathrm{~atm}$, and residence time $1.5 \mathrm{~s}$. The surrogate RME and SME compositions are taken from Table 13 [145].

In the continuity of their studies on MB [146] and MD [147], Saggese et al. [148] developed a lumped kinetic model for the pyrolysis and oxidation of biodiesel fuels. This model contains about 420 
species involved in approximately 13,000 reactions. Data computed with the lumped model were compared with data computed with the one proposed by Westbrook et al. [145] and experimental data from the literature $[194,195,197-199]$ showing a reasonable agreement.

\subsection{Ethyl ester kinetic investigations}

Research related to the oxidation of fatty acid ethyl esters (FAEE) is scarce, likely because commercial biodiesel components still consist of fatty acid methyl esters (FAME). Consequently, current research is still at the mechanism development stage for small model molecules that are known to be poor biodiesel surrogates but will provide significant information on ethyl ester oxidation behavior. In addition, the few published studies present oxidation results for FAEE by comparison with the studies for FAME, which highlights the advantages and disadvantages of both biodiesel alternatives as fuels. Therefore, the same approach will be adopted in this review for presenting the investigations in an effective way. The results will be first presented for ethyl propanoate and ethyl butanoate (versus methyl butanoate) and then for ethyl nonanoate (versus methyl nonanoate).

The original tendency was to compare esters with the same chemical formula (isomers) while varying the length of the alkyl and aliphatic chains to investigate the effect of the molecular structure on the oxidation chemistry. Once different mechanisms were elucidated for various alkyl esters, the studies were devoted to compare the oxidation behavior of the functional groups, methyl esters and ethyl esters, for molecules with the same aliphatic main chain length.

\subsubsection{Ethyl propanoate versus methyl butanoate}

Schwartz et al. [165] studied five different isomer esters with the chemical formula $\mathrm{C}_{5} \mathrm{H}_{10} \mathrm{O}_{2}$, including methyl butanoate $(\mathrm{MB})$, methyl isobutyrate (MiBu), ethyl propanoate (EP), propyl acetate (PrAc), and isopropyl acetate (iPrAc). Their experiments were performed at atmospheric pressure in methane/air coflowing non-premixed flames separately doped with 5000 ppm of each ester (Table 4). These flames are typical of many practical combustors, especially soot-producing systems, such as diesel engines and gas turbines, and yet simple enough to permit a basic understanding. The mole fractions of the major flame species were measured using electron impact mass spectrometry. The authors determined that the main decomposition pathway in non-premixed flames for EP, as for acetate esters ( $\operatorname{PrAc}$ and $\mathrm{iPrAc}$ ), was a unimolecular-six-centered dissociation reaction, which led to a carboxylic acid and a 1-alkene (propanoic acid and ethylene for EtPr, eq. (4)). However, MB and $\mathrm{MiBu}$, which cannot undergo a unimolecular-six-centered dissociation reaction, had decomposition rates that were consistent with a unimolecular $\mathrm{C}-\mathrm{O}$ fission mechanism, which generated two radicals (eq. (5) for $\mathrm{MB}$ ).

$\mathrm{C}_{2} \mathrm{H}_{5} \mathrm{C}(\mathrm{O}) \mathrm{C}_{2} \mathrm{H}_{5} \rightarrow \mathrm{C}_{2} \mathrm{H}_{5} \mathrm{C}(\mathrm{O}) \mathrm{OH}+\mathrm{C}_{2} \mathrm{H}_{4}$

$\mathrm{C}_{3} \mathrm{H}_{7} \mathrm{C}(\mathrm{O}) \mathrm{CH}_{3} \rightarrow \mathrm{C}_{3} \mathrm{H}_{7} \mathrm{C}(\mathrm{O}) \mathrm{O}+\mathrm{CH}_{3}$

The authors also observed propene (whose presence correlates to the formation of aromatics and soot) as a major decomposition product for all esters investigated. Nevertheless, EP and MB produced the lowest concentrations of propene, which is in agreement with the suggested primary reaction pathways (eqs. (4) and (5)).

Simultaneously to MB (Section 3.4.1), Metcalfe et al. [167] also studied the oxidation of EP in a shock tube. For $\mathrm{EP} / \mathrm{O}_{2} / \mathrm{Ar}$ mixtures, ignition delay times were measured behind the reflected-shock waves over a temperature range of 1140-1675 $\mathrm{K}$ at two low reflected-shock pressures and various equivalence ratios, including fuel-lean and fuel-rich conditions (Table 4). The authors reported that as EP concentrations were increased (from 1 to $1.5 \%$ ) with the $\mathrm{O}_{2}$ concentration constant (at $6.5 \%$ ), 
ignition delay times increased. Conversely, increasing $\mathrm{O}_{2}$ concentrations (from 6.5 to $26.0 \%$ ) with a constant EP concentration (of $1 \%$ ) led to a significant reduction in delay times. The authors noted that this negative power dependence of $\mathrm{O}_{2}$ was in accordance with previous work on hydrocarbons [217]. Furthermore, because the chain-branching mechanism at the high temperatures investigated in their work (1140-1675 K) is due to the $\mathrm{H}+\mathrm{O}_{2}=\mathrm{O}+\mathrm{OH}$ reaction, fuel-lean mixtures are more reactive in this regime (however, at low temperatures, because chain-branching is dependent on radical species formed directly from the parent fuel, fuel-rich mixtures are oxidized more quickly).

Metcalfe et al. [167] also observed that EP was consistently faster to ignite than MB, particularly at low temperatures. Theoretical interpretation was applied to this behavior based on the detailed chemical kinetic mechanism they developed for the EP combustion. This mechanism contained 139 species and 786 reversible reactions, and the EP submechanism was built by analogy with MB (Table 5). However, some changes were made, which are mentioned in the following.

- The six-centered unimolecular elimination reaction that produces propanoic acid and ethylene, as previously stated by Schwartz et al. [165] (eq. (4)), has been added and characterized by an activation energy of approximately $50 \mathrm{kcal} \mathrm{mol}^{-1}$ (Fig. 20).

- The EP mechanism contained the recently published $\mathrm{H}_{2} / \mathrm{O}_{2}$ submechanism by O'Conaire et al. [224].

- As for the MB mechanism, unimolecular decomposition reactions were treated to account for pressure falloff.

- The ethylene submechanism was based on a previously published mechanism by Curran et al. [217].

- The authors developed a propanoic acid submechanism based on the $\mathrm{n}$-heptane and iso-octane kinetic mechanisms previously published by Curran et al. ([207,217], respectively).

These changes led Metcalfe et al. [167] to perform simulations in good agreement with the experimental data. Also, production rate analyses achieved with the proposed mechanisms led the authors to explain the faster reactivity of EP (compared to that of $M B$ ) by the six-centered unimolecular decomposition which has a relatively low activation energy barrier and produces propanoic acid and ethylene (Fig. 20). According to the authors, it is the faster reactivity of these two products that is responsible for EP behavior.

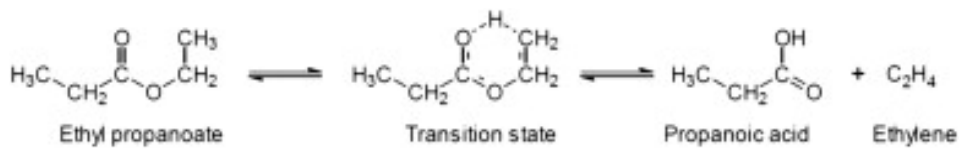

Figure 20. Six-centered unimolecular elimination for ethyl propanoate producing propanoic acid and ethylene [167].

Following this work for EP, Metcalfe et al. [202] recently performed JSR oxidation experiments that they used to further validate their previously proposed kinetic model [167]. Measurements were conducted under moderate pressure (10 atm), for various equivalence ratios and at temperatures in the range of 750-1100 K (Table 4). Fuel (EP), intermediate and final product species were recorded as a function of temperature. The authors reported that the main intermediate species observed were ethylene, propanoic acid, formaldehyde, and methane, and ethylene and propanoic acid were the most abundant, whereas the major products were $\mathrm{H}_{2} \mathrm{O}, \mathrm{CO}_{2}$, and $\mathrm{CO}$.

To obtain a better agreement with the profiles of the JSR species, the authors made some changes to the previously published EP oxidation mechanism [167].

- The rate constant for the unimolecular elimination reaction EP $=\mathrm{C}_{2} \mathrm{H}_{4}+\mathrm{C}_{2} \mathrm{H}_{5} \mathrm{COOH}$ was increased by a factor of four from $4.0 \times 10^{12} \exp \left(-50,000 /\right.$ RT) to $1.6 \times 10^{13} \exp (-50,000 / \mathrm{RT}) \mathrm{s}^{-1}$ (Ea in cal mol ${ }^{-1}$ ).

- The rate constants for three abstraction reactions were decreased by a factor of two. These are listed in Table 14 with updated values of the rate coefficients. 
- An updated $\mathrm{C}_{3}$ submechanism developed by the authors and collaborators was incorporated.

Table 14. Abstraction reactions for oxidation of ethyl propanoate as modified by Metcalfe et al. [202].

\begin{tabular}{cccc}
\hline Reaction & $\boldsymbol{A}$ & $\boldsymbol{n}$ & $\boldsymbol{E}_{\boldsymbol{a}}$ \\
\hline $\mathrm{EP}+\mathrm{HO}_{2}=\mathrm{H}_{2} \mathrm{O}_{2}+\mathrm{CH}_{3} \mathrm{CH}(\mathrm{CO}) \mathrm{OC}_{2} \mathrm{H}_{5}$ & $2.16 \mathrm{E}+12$ & 0.0 & 14,400 \\
$\mathrm{EP}+\mathrm{HO}_{2}=\mathrm{H}_{2} \mathrm{O}_{2}+\mathrm{CH}_{3} \mathrm{CH}_{2}(\mathrm{CO}) \mathrm{OCHCH}$ & $3.61 \mathrm{E}+03$ & 2.5 & 10,530 \\
$\mathrm{C}_{2} \mathrm{H}_{5} \mathrm{COOH}+\mathrm{HO}_{2}=\mathrm{H}_{2} \mathrm{O}_{2}+\mathrm{CH}_{3} \mathrm{CHCOOH}$ & $2.16 \mathrm{E}+12$ & 0.0 & 14,400 \\
\hline
\end{tabular}

${ }^{\mathrm{a}}$ The rate coefficients are listed in the generalized Arrhenius form $\left(\mathrm{k}=\mathrm{AT} \mathrm{T}^{\mathrm{n}} \cdot \exp (-\mathrm{Ea} / \mathrm{RT})\right)$ where units are mol, $\mathrm{cm}, \mathrm{s}, \mathrm{cal}$, and $\mathrm{K}$.

The revised mechanism was reported to be in good agreement with experiments (performed in JSR during this last study [202] and in an ST during the previous study [167]). Nevertheless, the authors observed better performance at stoichiometric and lean conditions than at rich conditions. In addition, the rate of production analysis led the authors to observe that the elimination reaction played a much smaller role in the decomposition of EP relative to $\mathrm{H}$-abstraction under the JSR conditions compared to the ST ones. Furthermore, the sensitivity analysis highlighted the importance of ethylene chemistry on the overall reactivity of the system.

Walton et al. [171] also performed low-temperature ignition of EP using an RCM (Table 4). The authors confirmed the observation made by Schwartz et al. [165] and Metcalfe et al. [167], according to which, EP ignited more rapidly than MB under the investigated conditions. The authors provided the same explanation of this reactivity as Metcalfe et al. [167] through the faster unimolecular decomposition of EP, which led to the formation of ethylene and propanoic acid. In addition, Walton et al. [171] proposed a new mechanism for EP oxidation based on the Metcalfe et al. [167] model that they improved to more closely match the experiments, particularly at low temperatures. The modified reactions and rate constants are summarized in Table 15.

Table 15. Reactions modified by Walton et al. [171] from the Metcalfe et al. mechanism [167] for EP oxidation. ${ }^{\mathrm{a}}$

\begin{tabular}{lllc}
\hline \multicolumn{1}{c}{ Reaction } & $\boldsymbol{A}$ & $\boldsymbol{n}$ & $\boldsymbol{E}_{\boldsymbol{a}}$ \\
\hline $\mathrm{CH}_{3} \mathrm{CH}_{2}(\mathrm{CO}) \mathrm{OC}_{2} \mathrm{H}_{5}+\mathrm{HO}_{2}=\mathrm{H}_{2} \mathrm{O}_{2}+\mathrm{CH}_{2} \mathrm{CH}_{2}(\mathrm{CO}) \mathrm{OC}_{2} \mathrm{H}_{5}$ & $8.30 \mathrm{E}+03$ & 2.55 & 16,490 \\
$\mathrm{CH}_{3} \mathrm{CH}_{2}(\mathrm{CO}) \mathrm{OC}_{2} \mathrm{H}_{5}+\mathrm{HO}_{2}=\mathrm{H}_{2} \mathrm{O}_{2}+\mathrm{CH}_{3} \mathrm{CH}(\mathrm{CO}) \mathrm{OC}_{2} \mathrm{H}_{5}$ & $1.50 \mathrm{E}+12$ & 0.00 & 14,400 \\
$\mathrm{CH}_{3} \mathrm{CH}_{2}(\mathrm{CO}) \mathrm{OC}_{2} \mathrm{H}_{5}+\mathrm{HO}_{2}=\mathrm{H}_{2} \mathrm{O}_{2}+\mathrm{CH}_{3} \mathrm{CH}_{2}(\mathrm{CO}) O \mathrm{CHCH}_{3}$ & $2.50 \mathrm{E}+03$ & 2.55 & 10,530 \\
$\mathrm{CH}_{3} \mathrm{CH}_{2}(\mathrm{CO}) \mathrm{OC}_{2} \mathrm{H}_{5}+\mathrm{HO}_{2}=\mathrm{H}_{2} \mathrm{O}_{2}+\mathrm{CH}_{3} \mathrm{CH}_{2}(\mathrm{CO}) \mathrm{CCH}_{2} \mathrm{CH}_{2}$ & $8.30 \mathrm{E}+03$ & 2.55 & 16,490
\end{tabular}

${ }^{a}$ The remainder of the mechanism was unchanged from Metcalfe et al. mechanism [167]. The rate coefficients are listed in the generalized Arrhenius form $(\mathrm{k}=\mathrm{ATn} \cdot \exp (-\mathrm{Ea} / \mathrm{RT}))$ where units are $\mathrm{mol}, \mathrm{cm}, \mathrm{s}, \mathrm{cal}$, and $\mathrm{K}$.

\subsubsection{Ethyl butanoate versus methyl butanoate; ethyl pentanoate versus methyl hexanoate}

In a comparative study relating the oxidation chemistry of methyl and ethyl ester groups for which $\mathrm{MB}$ results have been previously reported (Section 3.4.1), Hakka et al. [174] investigated the autoignition of ethyl butanoate (EB) behind reflected-shock waves by setting the same operating conditions for both esters (Table 4). The results showed that ignition delay times increased when equivalence ratios were increased, which is in agreement with the Arrhenius-type empirical equation obtained by the authors, by correlating statistically the ignition delays versus temperature and concentrations in oxygen and fuel

$\tau(\mathrm{s})=1.88 \times 10^{-28} \cdot \exp (57,540 / \mathrm{RT}) \cdot[\mathrm{EB}]^{0.250} \cdot[\mathrm{O} 2]^{-1.52}$

(with activation energy in $\mathrm{cal} \mathrm{mol}^{-1}$ and concentrations in $\mathrm{mol} \mathrm{cm}^{-3}$ ). The resulting equation also allowed the authors to note the obtained power dependences, which were strongly negative for $\mathrm{O}^{2}$ and small for EB. This phenomenon was also observed by Metcalfe et al. [167] for EP ignition in shock tubes. 
Regarding the comparative analysis between $\mathrm{MB}$ and EB ignition, Hakka et al. [174] observed small differences in reactivity between both esters, except above $1600 \mathrm{~K}$, where EB was observed to ignite slightly faster than MB. Furthermore, the higher reactivity of ethyl esters compared to methyl esters was also observed by Metcalfe et al. [167] for MB and EP but more prominently and particularly at lower temperatures. The difference in the extent of reactivity observed by both authors between $M B$ and $E B$ and $M B$ and $E P$ may be because, although all esters were in stoichiometric mixtures, $M B$ and $E P$ were introduced with the same molar fractions, whereas $M B$ and $E B$ having different molecular formula were not in the same molar fractions to maintain the same carbon atom concentration and $\mathrm{C} / \mathrm{O}$ ratio (simulating molecules of pseudo-identical chemical formula). In such circumstances, comparison of the oxidation behavior between $\mathrm{MB}$ and $\mathrm{EB}$ highlights the impact related to the methyl and ethyl ester functional groups, whereas the same comparison between MB and EP highlights not only the impact related to methyl and ethyl ester functional groups but also differences in aliphatic main chain length.

Hakka et al. [174] also proposed a detailed chemical kinetic mechanism for EB oxidation involving 115 species and 1101 reactions (Table 5). The mechanism was automatically generated using the same version of EXGAS software as the one used for MB [152,153,174]. Because validation through comparison of simulated and experimental results could be achieved by Hakka et al. [174] in shock tube conditions exclusively (because of the lack of other experimental information for EB oxidation at that time), this mechanism included only high-temperature reactions that were developed by analogy with MB. As reported by the authors, two significant changes resulted in differences with the $M B$ mechanism: first, the inclusion of the molecular elimination reaction similar to that reported for EP by Metcalfe et al. [167] (Fig. 20) but leading for EB to the formation of ethylene and butanoic acid (BA), and second, the secondary reactions of BA for which new rules of generation were adopted. Considering the molecular elimination characteristics to ethyl esters, which is favored thanks to the six-membered ring transition state it proceeds through (Fig. 21), the authors adopted an activation energy of $47.3 \mathrm{kcal} \mathrm{mol}^{-1}$ and an A-factor of $2 \times 10^{12} \mathrm{~s}^{-1}$ according to the measurements performed by Kairaitis and Stimson [242]. Regarding the secondary reactions of BA, these steps were automatically generated by considering this intermediate product as an initial reactant and developing a new detailed chemical kinetic submechanism for BA oxidation (built on a comprehensive primary mechanism and a secondary global chemical mechanism).

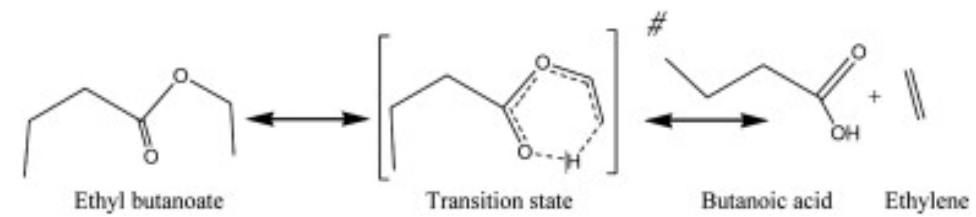

Figure 21. Six-centered unimolecular elimination reaction for ethyl butanoate producing butanoic acid and ethylene [174].

The classes of elementary reactions used to build the primary mechanism related to the hightemperature oxidation of carboxylic acids are similar to those used in the case of methyl esters. However, the kinetic parameters of bimolecular reactions related to $\mathrm{H}$-abstraction for the $\mathrm{O}-\mathrm{H}$ moiety of the carboxylic acid function were taken as equal to those of a tertiary alkylic $\mathrm{H}$-atom [149]. The unimolecular initiation involving the breaking of the $\mathrm{O}-\mathrm{H}$ bond was also accounted for by the authors. The simulation (conducted with SENKIN module of CHEMKIN software [158]) showed correct agreement between the experimental and modeling results.

In addition, reaction flux and sensitivity analyses helped the authors to further refine the arguments presented by Metcalfe et al. [167] for MB and EP oxidation behavior, according to which, the faster reactivity of the ethyl ester should be attributed to the faster reactivity of its oxidation products, 
such as carboxylic acid and ethylene. Hakka et al. [174] attributed the faster reactivity of EB compared to $\mathrm{MB}$ to the easier unimolecular initiation involving the production of branching agents $(\mathrm{H}$ radicals) through carboxylic acid formation (Fig. 22). Hence, the fact that this feature was observed particularly at high temperature (above $1600 \mathrm{~K}$ ) was explained by the increasing importance of the unimolecular initiations with temperature. The authors also denoted from these analyses that the six-centered unimolecular elimination from EB decreased when temperature increased (this channel representing $75 \%$ of the EB consumption at $1370 \mathrm{~K}$ against $40 \%$ at $1635 \mathrm{~K}$ ).

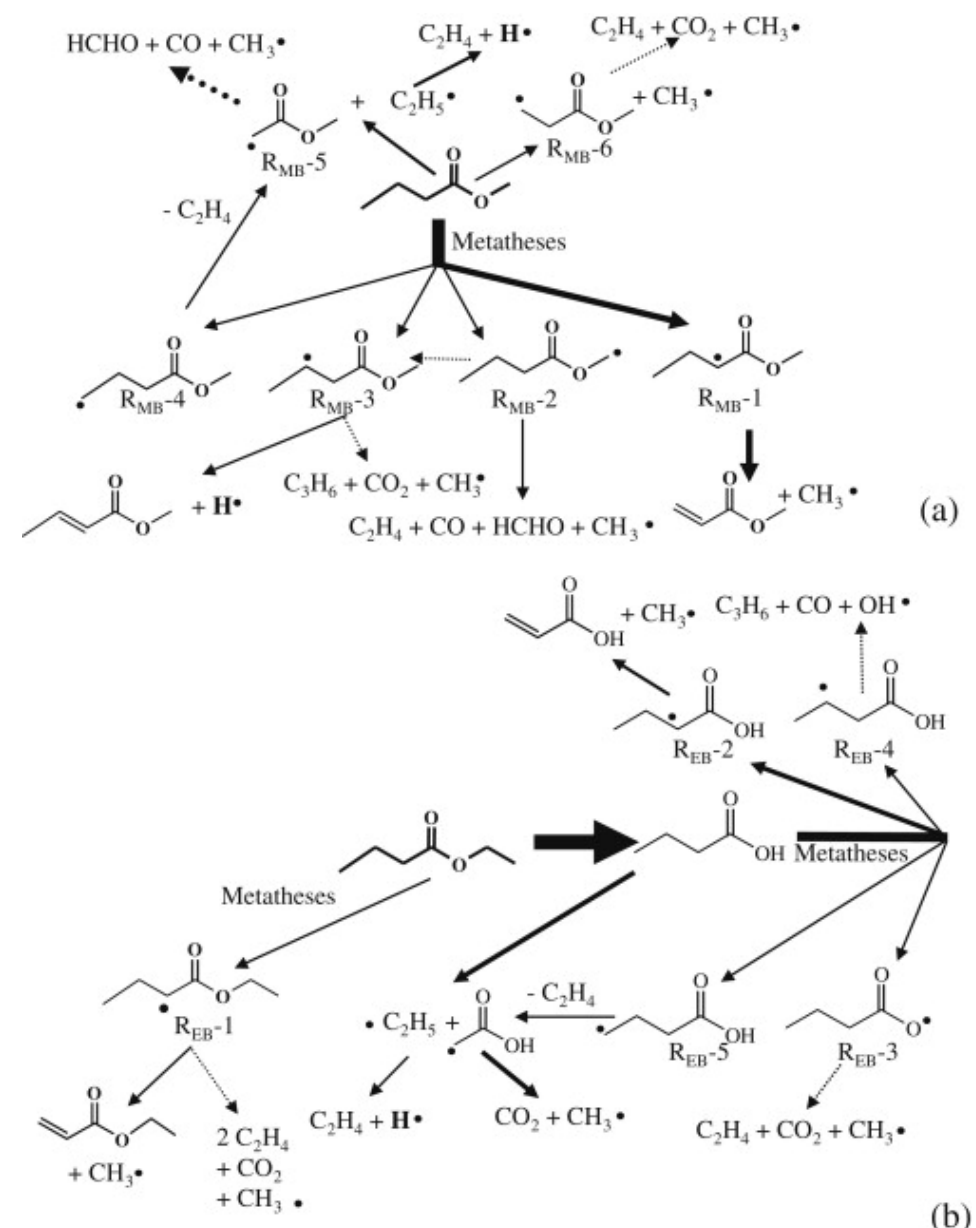

Figure 22. Reaction flux analysis performed at $1370 \mathrm{~K}$ for an equivalence ratio of 1 , and for $50 \%$ conversion of ester in the case of (a) methyl butanoate and (b) ethyl butanoate [174]. The size of the arrows is proportional to the relative flux. The channels involving a consumption of the esters below $5 \%$ are not shown. Dotted arrows represent several successive elementary reactions.

These modeling results, including reaction flux and sensitivity analyses, were confirmed recently at medium and low temperatures by Bennadji et al. work [203] devoted to EB oxidation in a laminar tubular plug-flow reactor (PFR). The new experimental information (concentration profiles of the reactants, stable intermediates, and final products) was generated at atmospheric pressure over the temperature range of 500-1200 $\mathrm{K}$ and under various dilutions, equivalence ratios, and residence times (Table 4). The authors used the model by Hakka et al. [174] without any significant modification for simulating the generated PFR data using the PSR module of the CHEMKIN computer package [158] (Table 5). The tubular PFR was modeled using a number of equivalent continuous perfectly stirred tank reactors (ePSR) in series, each uniform in composition, pressure, and temperature (the latter being attributed according to the temperature profile measured along the tubular PFR for each experiment). Bennadji et al. [203] reported a general agreement between the 
experimental and simulated results, which confirmed the validity of the model by Hakka et al. [174], although it was primarily performed based on high-temperature oxidation reactions. Bennadji et al. [203] also reported several additional features under the investigated conditions, briefly presented in the following.

- EB oxidation began at $800 \mathrm{~K}$ (with a significant reactivity in the 900-1000 K range) for fuel-lean mixtures and for fuel-rich mixtures. In addition, BA and ethylene were the major products without observing ethyl acrylate formation (indicating that few $\mathrm{H}$-abstractions would occur on the $\mathrm{EB}$ aliphatic main chain). These experimental observations, as well as the reaction flux and sensitivity analyses conducted on the PFR, led the authors to confirm that the main pathway of EB oxidation is the six-centered unimolecular elimination reaction that leads to ethylene and $\mathrm{BA}$. Then, $\mathrm{BA}$ is almost entirely consumed by $\mathrm{H}$-abstraction with $\mathrm{H}, \mathrm{OH}, \mathrm{HO}_{2}$, and $\mathrm{CH}_{3}$ radicals (metatheses), followed by $\beta$-scission reactions to mainly produce acrylic acid through an REB-2 radical, as shown in Fig. 22b. This pathway is favored by the weakness of the $\mathrm{C}-\mathrm{H}$ bond in the $\alpha$-position to the carboxylic acid chemical function.

- High concentrations of $\mathrm{CO}$ coupled with low concentrations of $\mathrm{CO}_{2}$ were obtained under fuel-rich conditions, whereas the reverse situation was observed under fuel-lean conditions. Hence, the fraction of $\mathrm{CO}_{2}$ resulting from $\mathrm{CO}$ oxidation is added to the $\mathrm{CO}_{2}$ formed by decarboxylation of $\mathrm{BA}$.

- In test conditions, decarboxylation of BA occurred at approximately $950 \mathrm{~K}$, with almost complete consumption of BA at $1000 \mathrm{~K}$, which suggests that BA decarboxylation should not occur until its aliphatic main chain has been largely consumed and incorporated oxygen in the molecule (similarly to large methyl esters as suggested by Szybist et al. [186] and [187] for MD oxidation).

- No NTC behavior was observed for EB, which is similar to MB [167] and [202] and demonstrates that $E B$ is not an ideal surrogate molecule for a detailed study of ethyl biodiesel combustion; however, it should be regarded as a suitable model molecule for gaining insight into the oxidation chemistry of the ethyl ester functional group.

Dayma et al. [204] performed the experimental and modeling study of the oxidation of ethyl pentanoate (also named ethyl valerate) in a JSR and in a spherical combustion chamber. JSR data obtained in this study were compared with experimental data obtained for $\mathrm{MHX}$ in similar conditions in the high-temperature region. Very similar reactivities were obtained for the two species in the contrary of smallest esters $[167,174]$.

\subsubsection{Oxidation of model molecules suitable as biodiesel surrogates}

Given how investigations were conducted for methyl esters, researchers have realized that, although the work developed for the short esters has greatly helped the understanding of the chemistry of oxidation of the alkyl esters, only esters with long-aliphatic main chains exhibit the cool flame behavior that is characteristic of biodiesels. Hence, investigations moved forward directly from ethyl butanoate to long-aliphatic main chain ethyl esters.

These investigations were not directed to the development of detailed chemical kinetic mechanisms for the oxidation of long-aliphatic main chain ethyl esters but rather to the generation of experimental information focused on applications related to modern engine designs, and from which it was possible, to propose major reaction pathways. This is the subject of the following subsections illustrated for ethyl hexanoate and ethyl nonanoate.

\subsubsection{Ethyl hexanoate versus methyl heptanoate}

To examine the applicability of $\mathrm{C}_{8} \mathrm{H}_{16} \mathrm{O}_{2}$ ethyl and methyl esters as biodiesel surrogates for application in modern engine designs that employ low-temperature combustion strategies, Zhang and Boehman [205] performed an experimental study of the autoignition of ethyl hexanoate (EHX) and methyl heptanoate $(\mathrm{MH})$ in a motored Cooperative-Fuel Research engine. For each test fuel, while operating 
under fuel-lean conditions (equivalence ratio of 0.25 ) and at $600 \mathrm{rpm}$, the engine compression ratio (CR) was gradually increased from the lowest point (4.43) to the point where significant HTHR occurred. In addition, to draw the major low-temperature oxidation pathways for the two esters, the engine exhaust was sampled and analyzed using GC-MS and GC-FID/TCD at various CRs for which only LTHR occurred (Table 4).

From the heat release analyses, the authors observed that $\mathrm{EHX}$ and $\mathrm{MH}$ exhibited an evident cool flame and experienced a transition from single-stage LTHR to two-stage ignition with the increase of the engine $C R$, which led to the conclusion that these two esters are likely suitable biodiesel surrogates in terms of low-temperature oxidation characteristics. Furthermore, at a given CR, EHX was determined to have a later onset and lower magnitude of LTHR compared to $\mathrm{MH}$, which indicated that EHX is less reactive in the low-temperature region compared to $\mathrm{MH}$ (Fig. 23). The authors attributed this feature to the different aliphatic main chain lengths between the two esters.

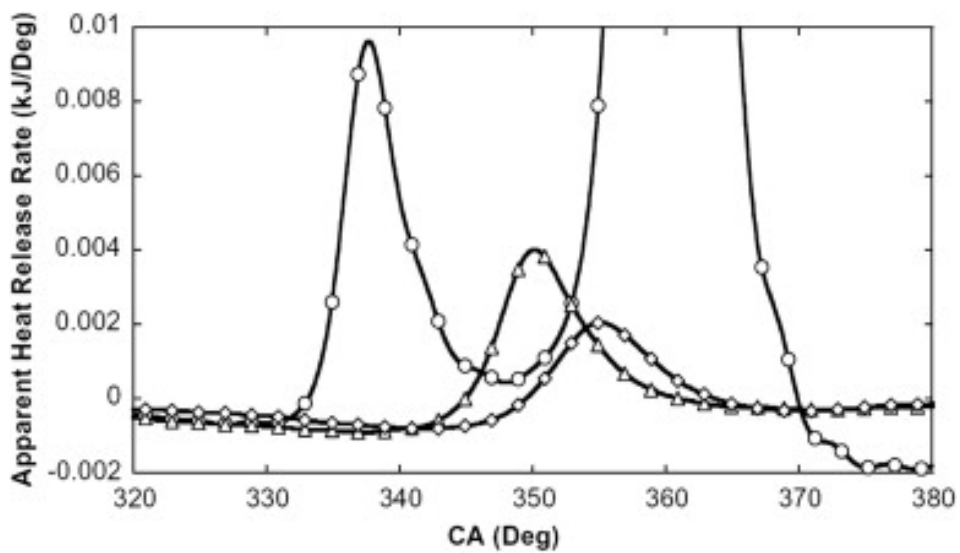

Figure 23. Comparison in heat release profiles between methyl heptanoate (triangle) and ethyl hexanoate (rhombus) at $C R=7.05$ [205]. n-Heptane (round) was also introduced by the authors for illustrating that the presence of ester moiety inhibits low-temperature reactivity. Indeed, n-heptane and methyl heptanoate have the same carbon chain length and yet $n$-heptane experiences two-stage ignition while both esters only exhibit single-stage at the operating CR.

Based on the literature mentioned above, this result appears to be in contradiction with the experimental and simulated observations previously performed by other researchers on the oxidation of EP and MB at high and low temperatures [165,167,171,202]. Hakka et al. [174] reported intermediary conclusions during their comparative study relating autoignition behavior between $E B$ and $\mathrm{MB}$ (both esters reacted equivalently from 1280 to $1600 \mathrm{~K}$; however, beyond this temperature range, $E B$ reactivity was determined to be more prominent). Similar to $E P$ and $M B, E H X$ differs from $\mathrm{MH}$ in one $\mathrm{CH}_{2}$ group on the aliphatic main chain in addition to the nature of the ester functional group (ethyl or methyl ester group). Nevertheless, a significant difference between EP and MB and $\mathrm{EHX}$ and $\mathrm{MH}$ is that the first class of esters accounts for short esters, whereas the second class accounts for long esters; however, no evident relationship between this structure-based feature and reactivity with a critical length of the aliphatic main chain has been highlighted. Moreover, the differences between the chemistry of oxidation at low and high temperatures should also be accounted for in this evaluation. As a result, it appears that understanding reactivity order between ethyl and methyl esters against temperature requires more investigation.

Concerning the results obtained by Zhang and Boehman [205] relating the exhaust species produced under engine conditions where only LTHR occurred, GC analyses showed that the aliphatic main chain of the two esters EHX and $\mathrm{MH}$ experienced the typical paraffin-like low-temperature oxidation sequence with the formation of unsaturated esters, epoxy esters, oxo-esters, aldehydes, and 
carboxylic acids. Similar to Dayma et al. [182], the authors also observed that the abstraction of $\mathrm{H}$ atoms on the $\alpha$-carbon of the ester carbonyl group, further involving the cleavage of the $\mathrm{C}-\mathrm{C}$ bond $\gamma$ to the ester carbonyl group to form alkyl (ethyl or methyl) propanoate, played an important role in the oxidation of long-aliphatic main chain esters. Moreover, in the case of EHX oxidation, a higher concentration of ethylene was observed compared to $\mathrm{MH}$, with the additional formation of hexanoic acid, all confirming the existence of the six-centered unimolecular elimination reaction during lowtemperature oxidation of ethyl esters as previously reported by Schwartz et al. [165], Metcalfe et al. $[167,202]$, and Walton et al. [171]. Based on these observations, the authors proposed major lowtemperature oxidation pathways for EHX (Fig. 24). As expected, this scheme is similar to the one adapted from Curran et al. [217] and Herbinet et al. [142] for the high- and low-temperature oxidation of methyl esters (Fig. 4). The difference is the insertion in the low-temperature channels of the unimolecular decomposition of the $\mathrm{RH}$ ester into a carboxylic acid and ethylene as well as with two additional decomposition paths of the hydroperoxy alkyl-ester radicals $(\mathrm{QOOH})$, which were prominent at intermediate temperatures and formed either unsaturated esters with a terminal double bond, aldehydes and hydroxyl radicals, or oxo-esters and hydroxyl radicals. These two last reaction pathways were proposed with slight differences by Bax et al. [195] for MO oxidation.

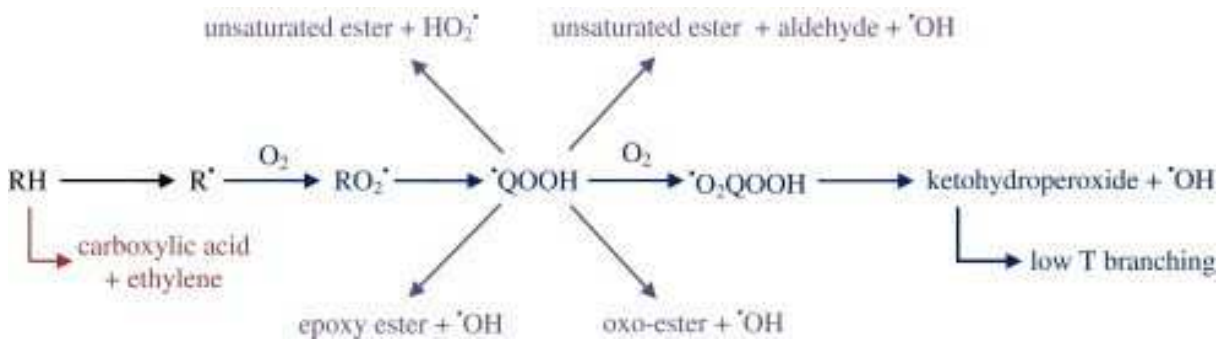

Figure 24. Major low-temperature oxidation pathways of ethyl hexanoate [205].

\subsubsection{Ethyl nonanoate versus methyl nonanoate}

To gain insight into the low-temperature oxidation of fatty acid esters produced from different alcohols with unsaturation sites located at different positions in the aliphatic main chain, Zhang et al. [1] experimentally investigated with the same engine environment as that used by Zhang and Boehman [205] the premixed ignition behavior of four $C_{9}$ fatty acid esters: methyl and ethyl nonanoate ( $\mathrm{MN}$ and $\mathrm{EN}$, respectively) together with two monounsaturated methyl esters, methyl-2and methyl-3-nonenoate (MN2EN and MN3EN, respectively) (Fig. 25). Attention was focused on understanding the primary reaction pathways responsible for early $\mathrm{CO}_{2}$ production during the lowtemperature oxidation of fatty acid esters, as this process is directly linked to the reduction of soot formation. This point is discussed in further details later on (Section 4.1).

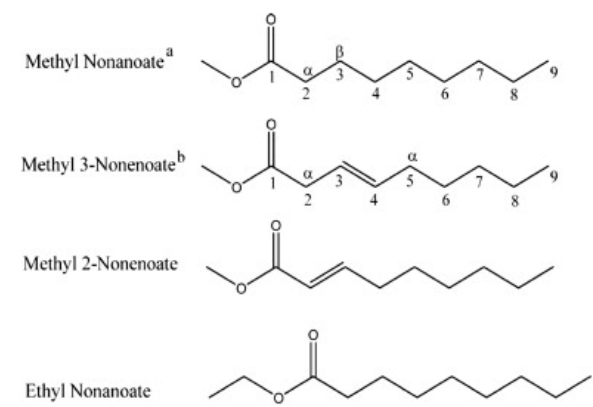

Figure 25. Molecular structures of the four $C_{9}$ esters investigated by Zhang et al. [1]. ${ }^{\text {T}}$ The carbon atom of the carbonyl group is carbon no. 1. Carbon no. 2 corresponds to the $\alpha$-carbon of the carbonyl group. ${ }^{\text {b}}$ The $\alpha$-carbons of the ethylenic bond correspond to the carbon no. 2 and carbon no. 5 for methyl-3-nonenoate. 